\title{
DEVELOPMENT OF NEW COMPUTATIONAL METHODS FOR NON-INVASIVE BRAIN STIMULATION
}

\author{
Dissertation \\ for the award of the degree \\ "Doctor of Philosophy" \\ Division of Mathematics and Natural Sciences \\ of the Georg-August-Universität Göttingen
}

within the doctoral program Theoretical and Computational Neuroscience of the Georg-August University School of Science (GAUSS)

submitted by

Alexander Opitz

from Erlangen, Germany

Göttingen, 2014 
Examination Board:

Prof. Dr. med. Walter Paulus (Supervisor, First Referee)

Georg August University Göttingen

Abteilung Klinische Neurophysiologie

Universitätsmedizin Göttingen

Robert-Koch-Straße 40, 37075 Göttingen

Prof. Dr. Florentin Wörgötter (Second Referee)

Georg August University Göttingen

Bernstein Center for Computational Neuroscience

Department for Computational Neuroscience

III Physikalisches Institut - Biophysik

Friedrich-Hund Platz 1, 37077 Göttingen

Prof. Dr. Jens Frahm

Georg August University Göttingen

Max-Planck-Institut für biophysikalische Chemie

Biomedizinische NMR Forschungs $\mathrm{GmbH}$

Am Fassberg 11, 37077 Göttingen

PD Dr. Peter Dechent

Georg August University Göttingen

Abt. Kognitive Neurologie

Universitätsmedizin Göttingen

Robert-Koch-Str. 40, 37075 Göttingen

Prof. Dr. Dario Farina

Georg August University Göttingen

Abt. Neurorehabilitation Engineering

Von-Siebold-Straße 4, 37075 Göttingen

Prof. Dr. Alexander Gail

Georg August University Göttingen

Deutsches Primatenzentrum

Sensorimotor Group

Kellnerweg 4, 37077 Göttingen

Date of oral examination: 


\section{Statement of Originality}

I hereby declare that this thesis is my own work and has been written independently with no

other sources and aids than quoted in the text, contributions, references and acknowledgements.

Göttingen, 24th April 2014 


\section{Table of Contents}

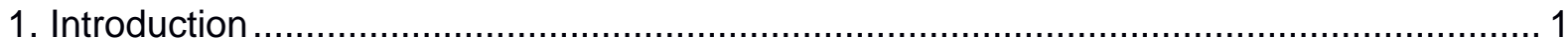

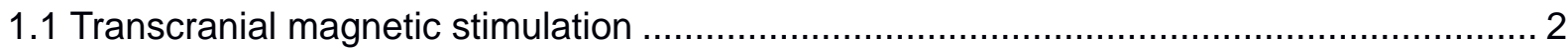

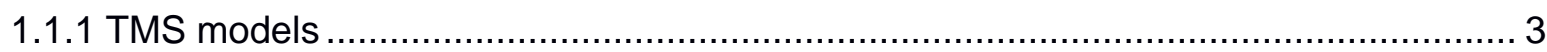

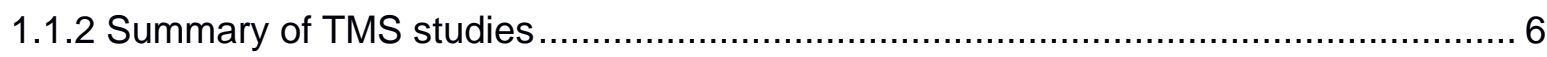

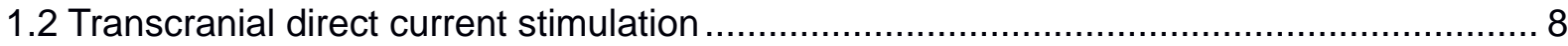

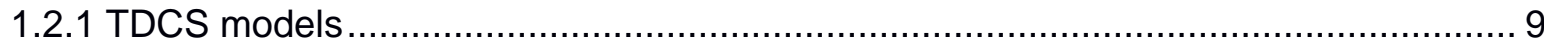

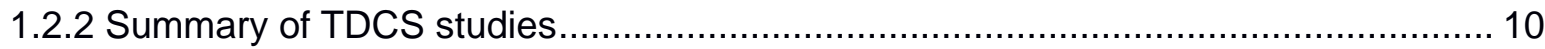

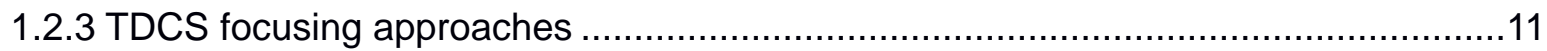

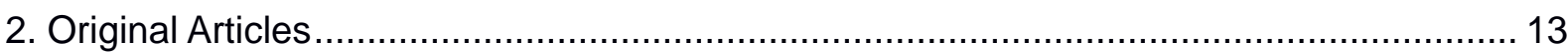

2.1 Physiological observations validate finite element models for estimating subject-specific electric field distributions induced by transcranial magnetic stimulation of the human motor cortex

2.2 Validating computationally predicted TMS stimulation areas using direct electrical stimulation in patients with brain tumors near precentral regions. 26

2.3 Anatomical determinants of the electric field during transcranial direct current stimulation: Anatomy may overrun electrode placement 34

3. Conclusion 59

Bibliography. 60

Acknowledgements. 66

CV. 67 


\section{Introduction}

Our brain works using electricity. Information between neurons is passed along their membranes by electrical currents in the form of action potentials. Action potentials are initiated when the electric potential between the intra- and extracellular space changes, crossing a certain threshold. Intact electrical processing is a necessary prerequisite for the functioning of the brain and various neurological and psychiatric disorders are thought to occur from pathological processes concerning the electrical signal propagation between neurons. It seems thus important to have tools available which allow us to interfere with electrical activity in the brain and study the effect of externally applied electric fields on neuronal activity.

However, the degree to which we can experimentally control and measure electric fields and currents varies strongly between different spatial scales. In patch clamp experiments, electrodes can be directly inserted into the cell and electric currents can be applied and measured at a closest possible distance to the neuron. Also in slice experiments, electric fields can be imposed in a very controlled manner at the cell culture, and the interaction between electric fields and neuronal activity can be measured. At the level of the human brain, a precise application and measurement of electric fields is more difficult as direct access to the brain is only given in the case of patients undergoing brain surgery, whereas in healthy subjects the skull and other tissues of the human head are major obstacles for electric fields reaching the brain. Nevertheless, in the last 20 - 30 years two methods to noninvasively apply electric fields to the brain emerged in the form of transcranial magnetic stimulation (TMS) and transcranial direct current stimulation (tDCS). Both methods work by imposing electric fields on the brain, either by placing a magnetic coil or electrodes on the scalp.

In this thesis, we explore the use of computational models that numerically solve the electric field during TMS and tDCS in order to improve the spatial precision of these methods. For TMS, we validate the prediction of those newly developed computational models with physiological measurements in healthy subjects as well as in brain tumor patients. In the following, we shortly describe the background of TMS and tDCS, as well as the current state of the art in computational models for brain stimulation, before outlining the new contributions to existing knowledge based on three publications/manuscripts. 


\subsection{Transcranial magnetic stimulation}

Transcranial magnetic stimulation works by placing a coil on the head which creates a temporally changing magnetic field, thus inducing an electric field in the brain (Barker et al., 1985). This electric field causes de- or hyperpolarization of neurons which can lead to the generation of action potentials when applied above a certain threshold. Over the motor cortex, TMS pulses can either elicit motor evoked potentials (MEPs) or, in the preinnervated muscle, induce a silent period. Only the motor cortex provides this easy cortical excitability readout that can be recorded electromyographically. TMS is used diagnostically e.g. to test the intactness of motor pathways (Rothwell et al., 1999). Furthermore, TMS is vastly employed in cognitive neuroscience research to investigate causal structure-function relationships in the brain (Pascual-Leone et al., 1999). The successive application of TMS pulses in a certain temporal order, so called repetitive TMS (rTMS) can, depending on the applied protocol, cause either inhibition or excitation of specific brain regions which are longer lasting and thought to be based on long term potentiation (LTP) or long term depression (LTD) like effects (Fitzgerald et al., 2006), and holds promise as a therapeutic tool for certain neuropsychiatric disorders (Padberg and George, 2009, Lefaucheur et al., 2011). Despite its widespread use and various applications, the exact neuronal mechanisms upon which TMS acts are still incompletely understood. Different possibilities through which TMS can excite neurons have been discussed in the literature. To cause a localized depolarisation of the neuronal membrane that can lead to the initiation of an action potential, the electric field has to show a spatial gradient along the neuron which was described with the so called activating function (Rattay, 1986). As the TMS induced electric field usually does not change strongly on the spatial scale of a neuron, it is rather the morphological changes in the neuron itself that determine the activation sites. One possible stimulation site could be axons bending in white matter (Amassian et al., 1992, Opitz et al., 2011, Nummenmaa et al., 2014). Also axonal or dendritic terminations can be subjected to stimulation (Salvador et al., 2011), thus enabling neurons that lie horizontally in the gyral crown (Day et al., 1989) or are aligned perpendicular to the sulcal wall (Fox et al., 2004) to be excited by strong tangential electric fields. Recently, it has been proposed that the soma is the main target of stimulation due to its thicker diameter and thus reduced threshold (Pashut et al., 2011). The importance of the direction of the electric field with respect to neuronal structures is clearly visible at the motor cortex. MEP amplitudes vary strongly depending on the orientation of the coil with respect to 
the motor cortex, with largest amplitudes being elicited when the electric field is aligned perpendicularly to the central sulcus (Brasil-Neto et al., 1992, Balslev et al., 2007, Richter et al., 2013). Currently, new TMS coil designs have been explored which might override the directional dependence of TMS to achieve a more uniform stimulation of neuronal populations (Rotem et al., 2014). It is still under discussion which elements of the motor cortex are the main contributors to a motor evoked potential. Both the "old M1" in the crown with disynaptic connections to the motor neurons or the "new M1" in the wall with monosynaptic connections (Rathelot and Strick, 2009) can potentially contribute to generate a MEP. From recordings in the pyramidal tract in cats and primates, two principal mechanisms for MEP generation are known. First, for high intensities an early short latency volley occurs which was termed "D - Wave" (direct), which is thought to result from a direct activation of pyramidal axons (Patton and Amassian, 1954). Second, later potentials, so called "I - Waves" (indirect), are elicited by cortical stimulation and transynaptically activate pyramidal tract neurons (Patton and Amassian, 1954). These findings were later confirmed with direct recordings in the spinal cord in awake patients (Di Lazzaro et al., 1998).

Irrespective of the neural mechanism of action that leads to excitation, the electric field is the main driving factor and a precise knowledge of the electric field distribution is of major interest to determine which part of the cortex is stimulated. Due to various different tissues in the head and the convoluted structure of the brain, the induced electric field follows an intricate pattern. As a direct measurement of the electric field in the brain is, at least in healthy subjects, not possible at the moment, computational models that numerically estimate the TMS induced electric field are widely applied. In the following, we give an overview of the computational models used in TMS and the underlying physical principles.

\subsubsection{TMS models}

The physical principle of TMS is based on Faraday's law of induction, that states that a changing magnetic field induces an electric field. This primary electric field is the main and driving force in TMS. In an inhomogeneous conductor, charges accumulate at tissue interfaces that create a secondary electric field. The equation that describes the dynamics of the TMS electric field is given by:

$$
\vec{E}=-\frac{\partial \vec{A}}{\partial t}-\nabla \varphi
$$


with $\vec{E}$ the induced electric field, $\vec{A}$ the magnetic vector potential from the TMS coil and $\varphi$ the scalar electric potential caused by charge accumulation at tissue interfaces (Thielscher et al., 2011). While analytical solutions exist for simple geometries, numerical methods have to be employed for more complex geometries like the human brain. The electric field distribution in a realistic model is determined by the electric properties of the tissues, which consist of the conductivity $\sigma$ and the relative permittivity $\varepsilon_{r}$. The conductivity $\sigma$ determines the ohmic current given by Ohm's law $\vec{J}=\sigma \vec{E}$, with the current density $\vec{J}$, conductivity $\sigma$, and electric field $\vec{E}$. The capacitive current is proportional to the relative permittivity $\varepsilon_{r}$. In the frequency range of TMS (1-10 kHz) the tissue can be described as purely resistive, as the ohmic current is much larger than currents involving the permittivity (Miranda, 2013). Typically, the ratio between the displacement current and the ohmic current is $\frac{\omega \epsilon}{\sigma} \ll 1$ for typical values of biological tissues in the frequency range of TMS (Foster and Schwan, 1989): $\sigma \sim 1 \mathrm{~S} / \mathrm{m}, \varepsilon \sim$ $10^{-6} \mathrm{~F} / \mathrm{m}$, for a frequency of $5 \mathrm{kHz}$. Thus, the dielectric properties of the tissue and the low frequencies make it possible for the simplifying quasistatic approximation to be used (Plonsey and Heppner, 1967). As the electromagnetic wavelength is many orders of magnitude larger than the human head, propagation effects can be neglected (Miranda, 2013). Also, magnetic fields that are produced by electric currents in the brain are not taken into account (Miranda, 2013). The quasistatic approximation allows us to decouple the spatial and the temporal components of the electric field, thus significantly simplifying the problem.

The accuracy of TMS simulation results strongly depends on the employed head model. Still widely used are spherical models (Roth et al., 1991, Heller and van Hulsteyn, 1992, Thielscher and Kammer, 2002, 2004) which remain popular due to their ease of implementation and computational efficiency (Salminen-Vaparanta et al., 2012, Nummenmaa et al., 2013) as well as their integration in neuronavigation systems (Ruohonen and Karhu, 2010). More detailed models take into account different tissues like white matter (WM), gray matter (GM), cerebrospinal fluid (CSF), skull and skin (Toschi et al., 2008, Salinas et al., 2009, Chen and Mogul, 2010). These models are based on magnetic resonance (MR) images from which the different tissues are segmented. From these segmentations a numerical model is created. One of the most popular numerical methods is the finite element method (FEM) which was first described for TMS by Wang and Eisenberg (1994). The FEM allows a volume to be discretized with small elements on which the electric field can be 
computed numerically. While hexahedral elements allow an easy implementation from segmented MR data, tetrahedral elements are more advantageous to approximate a convoluted surface like the cortex (Thielscher et al., 2011). Using a realistic model, it was shown that the electric field in GM is strongly dependent on the applied coil orientation (Thielscher et al., 2011). When the coil is oriented perpendicular to the cortex, the electric field in the gyral crown is enhanced compared to when the coil is oriented in parallel. The reason for this effect is that in the first case the current is traversing the highly conducting CSF before entering the GM while in the second case only to a smaller extent. This leads to an enhancement in the electric field in the lower conducting domain which is due to the required current preservation at the tissue interface (Miranda et al., 2003). The coil orientation that maximizes the electric field in the motor cortex also coincides with the empirically determined optimal coil orientation that results in strongest MEPs. In all these models, head tissues were treated as isotropic conductors, meaning that they do not show any preferential direction for current flow. However, WM in the brain is clearly anisotropic, where conductivities along large fiber bundles are up to ten times higher than perpendicular to them (Nicholson, 1965, Ranck and Bement, 1965). An estimate of WM conductivity can be achieved with diffusion MRI, as it was shown that the conductivity tensor can be estimated from the diffusion tensor (Tuch et al., 2001) because both diffusion and conduction are restricted by the same geometrical constraints. The incorporation of conductivity anisotropy for TMS simulations, resulted in high electric field strengths being found also in deeper WM regions (Opitz et al., 2011). The creation of realistic FEM models is computationally demanding and often needs a large amount of manual intervention, thus a widespread application is somewhat hampered. However, with increasing amount of automation in the model creation, the use of individually realistic FEM models is now feasible with moderate time investment (Windhoff et al., 2013). Realistic FEMs show promise to improve the targeting accuracy of TMS to optimize stimulation protocols and can also be used in conjunction with neuronavigation systems. Furthermore, interindividual variation in the response to TMS might potentially be explained by differences in the neuroanatomy between subjects.

So far, these models have not been used in experimental studies. Also validation studies are needed to evaluate if their more precise predictions compared to more simplified models, which are yet easier to implement, are of practical value. 
These questions were investigated in two projects validating computational models with physiological measurements. The results of these two papers are summarized and discussed in the following section.

\subsubsection{Summary of TMS studies}

In the first study "Physiological observations validate finite element models for estimating subject-specific electric field distributions induced by transcranial magnetic stimulation of the human motor cortex" published in Neurolmage, we combined realistic FEM models with physiologic MEP measurements. We demonstrate with a theoretical example, how a simple projection based approach to determine the stimulation area of TMS is highly volatile towards small tilts in the TMS coil. Furthermore, this approach cannot capture effects of coil rotations, as the projection point in the brain does not change when rotating the coil. In contrast, our FEM approach is robust towards small perturbations in the coil tilt and can accurately describe the effects of coil rotations. In a neuronavigated experiment we measured MEPs on a $5 \mathrm{~cm} \times 5 \mathrm{~cm}$ grid based on the motor cortex for two different coil orientations. Electric fields were computed with individualistic FEM models and a MEP weighted field distribution was calculated. It was demonstrated that this new method is able to reliably predict the motor cortex as the stimulation area. In a second step, directional electric field components, either perpendicular or tangential to the motor cortex in regions that showed strong fMRI BOLD effect during a voluntary finger movement task, showed strong correlations with the motor evoked potential. Our results show that realistic FEM models can indeed predict the physiological response induced by TMS and offer a robust tool to determine the cortical area that is subject to stimulation.

I co-designed the study, collected the MEP data, analyzed all data, created all figures and wrote the manuscript.

In a second study "Validating computationally predicted TMS stimulation areas using direct electrical stimulation in patients with brain tumors near precentral regions", in press in Neuroimage: Clinical, we validated computationally predicted TMS stimulation areas with direct electrical stimulation in tumor patients undergoing brain surgery. Motor evoked potentials were recorded before surgery on a grid based on the motorcortex as described in the previous study. Using individual realistic FEM models, we determined the TMS 
stimulation area based on the MEP-weighted electric field. In addition, we repeated the same computations with a spherical head model. During brain surgery the patients' motor cortex was stimulated with direct electrical stimulation (DES) and MEPs were recorded from the same target muscle as during TMS. We show that the predicted TMS stimulation area overlaps with the "gold standard" DES stimulation area. With increasing electric field strengths the average overlap reaches up to 80\%. Realistic FEM models show better overlap compared to spherical models, irrespective of the chosen DES region of interest. We conclude that realistic FEM models can reliably predict eloquent motor regions even in the presence of pathological brain tissue and might be useful in the future for presurgical mapping.

I co-designed the study, collected the TMS MEP data, analyzed all data, created all figures and wrote the manuscript. Direct electrical stimulation during surgery was performed by the Department of Neurosurgery, University Medical Center, Göttingen. 


\subsection{Transcranial direct current stimulation}

Another method to stimulate the brain in a non-invasive manner is tDCS. TDCS can modulate cortical excitability (Nitsche and Paulus, 2000) and shows promise as an inexpensive alternative to rTMS to treat certain neurological and psychiatric disorders (Paulus, 2011). In its simplest and most common form, two large electrodes (with several $\mathrm{cm}$ edge length) are attached to the scalp and a weak direct current usually between 1mA - 2mA is passed through them for several minutes (Nitsche et al., 2008). Compared to TMS, much lower electric fields are employed in tDCS: while for TMS typical electric fields are in the order of $100 \mathrm{mV} / \mathrm{mm}$ (Miranda et al., 2007, Salvador et al., 2011), electric fields for tDCS are only up to $1 \mathrm{mV} / \mathrm{mm}$ (Datta et al., 2009, Salvador et al., 2010, Miranda, 2013). These low electric fields do not lead to action potential initiation but can nevertheless cause neuromodulatory effects. In rat cortical pyramidal neurons it was demonstrated that low frequency extracellular electric fields can induce neural entrainment by ephaptic coupling (Anastassiou et al., 2011). Similar effects have been found in hippocampal slices using frequencies from 10-100 Hz (Deans et al., 2007) as well as with pulsed electric fields (Francis et al., 2003). Also, evidence was found that electric fields $<0.5 \mathrm{mV} / \mathrm{mm}$ can influence neural activity in the neocortex (Frohlich and McCormick, 2010, Ozen et al., 2010). While most of these in vitro studies used low frequency alternating electric fields, a few studies investigated the effect of direct currents. Using electric fields $<4 \mathrm{mV} / \mathrm{mm}$ and $<10$ $\mathrm{mV} / \mathrm{mm}$ changes in spike timing was found in hippocampal slices (Bikson et al., 2004, Radman et al., 2007, Reato et al., 2010). The effect of the applied electric field depends on its orientation with respect to the neuronal morphology (Radman et al., 2009, Rahman et al., 2013) further complicating the prediction of which neuronal elements are stimulated. Furthermore, due to the convoluted cortex, current flow follows an intricate pattern with inflowing and outflowing currents taking place even in the same gyrus (Reato et al., 2013). A simplification commonly used in computational models is the "quasi-uniform" assumption which states that membrane polarization is linearly proportional to the local electric field magnitude (Bikson et al., 2013a). Thus, computational models trying to predict stimulation areas typically employ electric field magnitude as a surrogate measure for excitation. Nevertheless, electric field direction is an important factor which might explain why certain montages are effective and others not, depending on the position of the stimulation electrodes which determine the direction of the electric field (Nitsche and Paulus, 2000). 
The electric field during tDCS depends on several anatomical factors which are unique for a certain individual. Especially, considering the large interindividual variability in the outcome of established tDCS protocols (López-Alonso et al., 2014, Wiethoff et al., 2014), subject specific models which might capture certain aspects of the observed variability seem promising and have been suggested as a possibility to improve on existing protocols (Bikson et al., 2013b, de Berker et al., 2013). Below, existing computational models of tDCS are discussed as well as the underlying physical concepts.

\subsubsection{TDCS models}

Compared to TMS the underlying equation that describes the electric field in tDCS is simpler:

$$
\vec{E}=-\nabla \varphi
$$

with $\vec{E}$ the electric field and $\varphi$ the scalar electric potential (Miranda et al., 2009). The electric field is determined by the gradient of the electric potential. At the positions of the electrodes a boundary condition is applied, which fixes the potential at a specific value. In a typical tDCS stimulator, the potential difference between two electrodes is adjusted such that a certain current (e.g. $1 \mathrm{~mA}$ ) is passing through them (current controlled stimulation). Although a large overlap exists between FEM models applied in TMS and tDCS, certain differences are present that should be discussed. While in TMS the magnetic field passes the skull undisturbed and the exact representation of the skull in the model is not of outmost importance, in tDCS the skull is, due to its low conductivity, the major obstacle of currents reaching the brain and an exact representation is needed for valid results.

Various different computational models have been employed to study the electric field distribution during tDCS, starting with simple spherical models (Rush and Driscoll, 1968, Datta et al., 2008) which were useful to study the effects of electrode size on the current density. With these models it was shown that the commonly used way to define the dose of tDCS, by dividing the stimulation current intensity by the electrode area, is not appropriate for keeping current density constant in the brain (Miranda et al., 2009). Later studies used more realistic MR-derived head models (Datta et al., 2009, Sadleir et al., 2010, Miranda et al., 2013) to study the effect of a convoluted brain on the electric field distribution. Effects of white matter conductivity anisotropy have been investigated and show that electric fields are 
slightly enhanced in deeper WM structures when taking anisotropy into account (Suh et al., 2012). The importance of the skull has been investigated in various studies. It was shown that gross alterations in skull conductivity caused by skull holes or metal plates can cause a significant distortion of the current flow (Datta et al., 2010). The influence of different skull layers (spongy bone, compact bone) was studied in various papers (Neuling et al., 2012, Rampersad et al., 2013, Wagner et al., 2013). In Rampersad et al. (2013) it was pointed out that the mean radial conductivity of the skull over the different layers influences the electric field distribution to a big extent. However, these papers either used more simplistic FEM models or concentrated on global effects of skull conductivity. Up until now it had not been studied how local skull thinnings affect the current distribution in the brain and how stimulation areas are influenced by various anatomical factors. We addressed this problem in one manuscript which is summarized below, using optimized FEM models for tDCS.

\subsubsection{Summary of TDCS studies}

In the study "Anatomical determinants of the electric field during transcranial direct current stimulation: Anatomy may overrun electrode placement" we studied how various anatomical factors determine the electric field distribution during tDCS. In a highly realistic FEM model we studied the most widely used electrode montage to induce motor plasticity. We varied the position of the stimulation electrode over the motor cortex in small steps (5mm) over an area of $4 \mathrm{~cm} \times 4 \mathrm{~cm}$. In addition, we investigated the effect of electrode rotations on the electric field distribution. Using a multiregression model we quantified the effect of skull thickness, sulcal depth and CSF thickness as well as distance to the electrode edge on the electric field distribution in a region around the motor cortex. All four factors exhibit a negative relationship with the electric field. Taken all factors together, we explain about $50 \%$ of the spatial variance in the electric field. In a second step we investigated which part in the brain is stimulated strongest over all electrode positions. For that we determined the area which was above $80 \%$ of the maximum electric field in each simulation and computed an "activation index" as the ratio of how often a certain brain area was stimulated over all electrode positions. We found that certain brain regions were activated in most of the cases, irrespective of exact electrode position. They corresponded with overlying skull thinnings which act as pathways for current to enter the brain due to their enhanced conductivity compared to neighboring skull regions. 
We confirmed our findings in a second subject showing the same effects. In both subjects regions of highest electric field strength were located in premotor areas excluding a direct excitation of the primary motor cortex. Our findings have clear implications for the practical use of tDCS and are also emphasizing the potential importance of individualistic models to determine stimulation areas.

I co-designed the study, performed the simulations, conducted the data analysis and wrote the manuscript. An optimized FEM skull model was provided by Axel Thielscher.

\subsubsection{TDCS focusing approaches}

Despite the strong dependence of the electric field on anatomical factors several methods have recently been proposed to achieve a more focal stimulation with tDCS. In a typically employed two electrode montage with large pads, the electric field distribution covers large areas of the brain (Miranda et al., 2013). Various attempts have been made to improve the focality of transcranial electrical stimulation systems. One possibility is to use a ring-like electrode montage with one stimulation electrode in the center and a surrounding return electrode (Datta et al., 2008). This was later implemented in practice with one anode and four surrounding cathodes spaced a few centimeters apart (Caparelli-Daquer et al., 2012, Kuo et al., 2013) and termed high-density tDCS. The basic principle of this electrode montage is that the surrounding return electrodes stop the current from flowing outside the stimulation area. Although spatially closer electrodes lead to higher shunting of current through the skin, this is offset by increased focality in the target region. The principle of high-density tDCS is confirmed with modeling as well as physiological data (Edwards et al., 2013). Another even more sophisticated alternative is to use an EEG-like electrode array and use a beamforming approach to adjust input currents, such that the electric field in a given target region is optimized (Dmochowski et al., 2011, Ruffini et al., 2014). It has yet to be experimentally tested if multielectrode approaches using optimization techniques can account for interindividual differences in anatomy and lead to more reliable results than the most commonly used two electrode systems. Focusing approaches are mainly limited to superficial brain areas, as the electric field strength drops off with distance to the electrodes. In the quasistatic regime it is not possible to focus electric fields in depth (for both magnetic and electrical stimulation), irrespective of the number of used electrodes (Miranda, 2013). 
This is because the occurring electromagnetic wavelengths (in the range of kilometers) are far larger than the structure on which one would like to focus them (in the range of centimeters/millimeters). This fundamental physical limitation does not exist for transcranial focused ultrasound (Tufail et al., 2010, Legon et al., 2014) with wavelengths in the millimeter range that can be employed to stimulate brain tissue in a more focal manner. In a homogenous medium, the maximum electric field always occurs at the surface (Heller and van Hulsteyn, 1992). Nevertheless, as the head is a heterogeneous conductor, focusing effects can occur also in deeper brain structures which are due to the unique conductivity contribution in this region (Opitz et al., 2011). 
2. Original Articles 


\title{
Physiological observations validate finite element models for estimating subject-specific electric field distributions induced by transcranial magnetic stimulation of the human motor cortex ${ }^{i s}$
}

\author{
Alexander Opitz a,b, Wynn Legon ${ }^{a}$, Abby Rowlands ${ }^{\text {a }}$, Warren K. Bickel ${ }^{\text {a,c }}$, \\ Walter Paulus ${ }^{\mathrm{b}}$, William J. Tyler ${ }^{\mathrm{a}, \mathrm{c}, \mathrm{d}, *}$ \\ a Virginia Tech Carilion Research Institute, Roanoke, VA 24015, USA \\ ${ }^{\mathrm{b}}$ Department of Clinical Neurophysiology, Georg-August-University, Göttingen, Germany \\ c Department of Psychiatry and Behavioral Medicine, Virginia Tech Carilion School of Medicine, USA \\ d School of Biomedical Engineering and Sciences, Virginia Tech, USA
}

\section{A R T I C L E I N F O}

\section{Article history:}

Accepted 18 April 2013

Available online 1 May 2013

\section{Keywords:}

Transcranial magnetic stimulation

Motor cortex

Electric field

Finite element model

\begin{abstract}
A B S T R A C T
Recent evidence indicates subject-specific gyral folding patterns and white matter anisotropy uniquely shape electric fields generated by TMS. Current methods for predicting the brain regions influenced by TMS involve projecting the TMS coil position or center of gravity onto realistic head models derived from structural and functional imaging data. Similarly, spherical models have been used to estimate electric field distributions generated by TMS pulses delivered from a particular coil location and position. In the present paper we inspect differences between electric field computations estimated using the finite element method (FEM) and projection-based approaches described above. We then more specifically examined an approach for estimating cortical excitation volumes based on individualistic FEM simulations of electric fields. We evaluated this approach by performing neurophysiological recordings during MR-navigated motormapping experiments. We recorded motor evoked potentials (MEPs) in response to single pulse TMS using two different coil orientations ( $45^{\circ}$ and $90^{\circ}$ to midline) at 25 different locations ( $5 \times 5$ grid, $1 \mathrm{~cm}$ spacing) centered on the hotspot of the right first dorsal interosseous (FDI) muscle in left motor cortex. We observed that motor excitability maps varied within and between subjects as a function of TMS coil position and orientation. For each coil position and orientation tested, simulations of the TMS-induced electric field were computed using individualistic FEM models and compared to MEP amplitudes obtained during our motormapping experiments. We found FEM simulations of electric field strength, which take into account subject-specific gyral geometry and tissue conductivity anisotropy, significantly correlated with physiologically observed MEP amplitudes $\left(\mathrm{r}_{\max }=0.91, \mathrm{p}=1.8 \times 10^{-5} \mathrm{r}_{\text {mean }}=0.81, \mathrm{p}=0.01\right)$. These observations validate the implementation of individualistic FEM models to account for variations in gyral folding patterns and tissue conductivity anisotropy, which should help improve the targeting accuracy of TMS in the mapping or modulation of human brain circuits.
\end{abstract}

(c) 2013 The Authors. Published by Elsevier Inc. All rights reserved.

\section{Introduction}

Transcranial magnetic stimulation (TMS) is becoming a widely implemented tool in neuroscience for modulating brain circuit activity and holds promise for treating some neuropsychiatric disorders (Lefaucheur et al., 2011; Padberg and George, 2009). The use of TMS in research and clinical applications has been somewhat limited by

\footnotetext{
is This is an open-access article distributed under the terms of the Creative Commons Attribution-NonCommercial-No Derivative Works License, which permits non-commercial use, distribution, and reproduction in any medium, provided the original author and source are credited.

* Corresponding author at: Virginia Tech Carilion Research Institute, Roanoke, VA 24015, USA. Fax: +1 5409853371.

E-mail address: wtyler@vt.edu (W.J. Tyler).
}

variable outcomes and improvement on its implementation is still required (Padberg and George, 2009; Wagner et al., 2007; Wassermann and Zimmermann, 2012). The basic biophysical mechanism of TMS is that a time-varying magnetic field induces an electric field in brain tissue (Opitz et al., 2011; Wagner et al., 2006). The resulting electric field strength and its spatiotemporal distribution are critical factors influencing the tissue volumes and brain circuits affected by TMS. Thus, accurate methods for estimating these brain volumes are crucial for optimizing TMS coil positioning and circuit targeting strategies. This is especially true when one desires to implement TMS to elicit repeatable physiological and behavioral outcomes.

Various strategies have been implemented to predict the brain regions influenced by TMS. These targeting methods include the use of 10-20 EEG positioning coordinates, group functional Talairach coordinates, or 
MR-guided neuronavigation systems (Sack et al., 2009). The basic premise of these targeting methods is that the volume of the brain stimulated resides directly underneath the center of the TMS coil. Hence, TMS coils are typically positioned such that the desired targeted cortical area resides in the direction of the coil axis (Sparing and Mottaghy, 2008) and that the distance from the coil on the scalp to the cortical area is minimized (Rusjan et al., 2010). Cortical areas stimulated by TMS have also been predicted by projecting the center of gravity $(\mathrm{CoG})$ measured at the scalp onto the cortex (Diekhoff et al., 2011; Weiss et al., 2012) or using spherical models to estimate the electric field distribution (Salminen-Vaparanta et al., 2012; Thielscher and Kammer, 2002). These approaches however, do not take into account critical principles related to tissue specific conductance or boundary effects.

Projection-based methods of TMS targeting rely on the fact that the magnetic vector potential is maximal directly beneath the center of the coil for the most widely implemented figure-eight TMS coils. This is not necessarily the case for the electric field generated by a TMS pulse however. The electric field $(\vec{E})$ induced by TMS is composed of two components, where $\vec{E}=-\frac{\partial \vec{A}}{\partial t}-\nabla \varphi$ with $\vec{A}$ being the magnetic vector potential and $\varphi$ being the scalar electric potential. The second component in the equation occurs due to charge accumulation at tissue interfaces. Charge accumulation and conductivity differences in tissues and their borders, for instance skin-skull, skull-cerebrospinal fluid, cerebrospinal fluid-gray matter, and gray matter-white matter interfaces, have been shown to introduce significant distortions to electric fields generated by TMS in the brain (Chen and Mogul, 2010; Salinas et al., 2009; Thielscher et al., 2011; Toschi et al., 2008). These subjectspecific electric field distortions are not accounted for by either conventional CoG projection approaches or spherical models. Therefore, although these methods have collectively proven useful for estimating areas of cortex affected by TMS, they can be improved upon. In fact, it has been recently suggested that finite element modeling approaches can offer improved estimates of the electric field generated by TMS by considering distortions unique to an individual (Opitz et al., 2011; Thielscher et al., 2011; Windhoff et al., 2013).

High-resolution simulations using the finite element method (FEM) make more specific predictions about the distribution of the electric field generated by TMS and, compared to spherical models or center of gravity $(\mathrm{CoG})$ estimations, are thought to provide a more accurate estimation of the brain volumes affected by it (Opitz et al., 2011; Thielscher et al., 2011; Windhoff et al., 2013). Since the generation of FEM simulations are time consuming and simulations using them is computationally demanding, broad applications of FEM approaches in clinical neuromodulation and research has been scarce. With increasing automation in model creation, the use of individualized FEM simulations for predicting brain regions influenced by TMS pulses is becoming more feasible (Windhoff et al., 2013). However, FEM simulations have not been validated by physiological investigations aimed at determining their functional accuracy. In the present study we found that individualized FEM simulations can be used to estimate electric field strengths and distributions for accurately predicting the excitation volumes generated by TMS in brain circuits. By comparing our observations to projectionbased and CoG approaches, we further show how FEM simulations of electric fields can help to improve the spatial targeting accuracy of TMS by accounting for individual neuroanatomical differences. We anticipate that the broadened implementation of subject-specific FEM field simulations will result in an increased consistency across observations when TMS is used to modulate or map brain circuits.

\section{Materials and methods}

\section{Subjects}

Five participants ( 3 males, 2 females, ages 23-36, mean $27.6 \mathrm{yr} \pm$ $5.5 \mathrm{yr}$ ) provided written informed consent to participate in the study.
None of the participants reported any history of neurological or musculoskeletal impairment and all were right hand dominant. All procedures were approved by the Institutional Review Board at Virginia Tech.

\section{Magnetic resonance imaging (MRI)}

Functional and anatomical images were collected at Virginia Tech Carilion Research Institute on a Siemens 3T MRI Trio TIM scanner using a 12 channel head matrix coil. A 3D T1-weighted magnetization-prepared rapid acquisition gradient echo sequence (MPRAGE) anatomical scan was acquired for each subject $(\mathrm{TR}=2600 \mathrm{~ms}, \mathrm{TE}=3.02 \mathrm{~ms}$, flip angle $\theta=8^{\circ}, \mathrm{FOV}=256 \times 256 \mathrm{~mm}, 176$ slices, $1.0 \mathrm{~mm}$ isotropic resolution, transverse plane). A 3D T2-weighted ( $\mathrm{TR}=11,990 \mathrm{~ms}$, $\mathrm{TE}=93 \mathrm{~ms}$, flip angle $\theta=120^{\circ}$, bandwidth $=219 \mathrm{~Hz} / \mathrm{Px}$, echo spacing $=9.34 \mathrm{~ms}$, Turbo Factor $=11, \mathrm{FOV}=256 \mathrm{~mm} \times 256 \mathrm{~mm}, 2 \mathrm{~mm}$ isotropic resolution) sequence was acquired in the sagittal plane. BOLD images were acquired using gradient-echo echo planar imaging $(\mathrm{TR}=2000 \mathrm{~ms}$, TE $=$ $30 \mathrm{~ms}$, flip angle $\theta=90^{\circ}$, FOV $=190 \mathrm{~mm}, 33$ slices, slice thickness $=$ $3 \mathrm{~mm}$ ). An additional higher resolution gradient-echo echo planar imaging sequence $\left(\mathrm{TR}=2000 \mathrm{~ms}, \mathrm{TE}=30 \mathrm{~m}\right.$, flip angle $\theta=50^{\circ}$, FOV $=$ $200 \mathrm{~mm} \times 200 \mathrm{~mm}, 20$ slices, slice thickness $=1.8 \mathrm{~mm}$ ) was collected in the transverse plane overlying the motor cortex.

Diffusion-weighted images using a spin echo EPI sequence (TR = $8700 \mathrm{~ms}, \mathrm{TE}=96 \mathrm{~ms}, 64$ axial slices, voxel size $=2 \times 2 \times 2 \mathrm{~mm}^{3}$, GRAPPA acceleration factor 2, 6/8 phase partial Fourier, 2 averages) with 64 diffusion directions with a b-value $1500 \mathrm{~s} / \mathrm{mm}^{2}$ and one $\mathrm{b}=0 \mathrm{~s} / \mathrm{mm}^{2}$ image were also acquired.

\section{Behavior}

In the MRI scanner, participants were required to perform four movements, which included adduction-abduction of their right index finger. Only the finger movement was used in this study. Movements were self-paced though encouraged to be performed at about $0.5 \mathrm{~Hz}$ unless fatigued. Participants were familiarized with the movements and allowed to briefly practice outside of the scanner. Movements were performed in four 40 second blocks interspersed by 40 second Rest blocks. Participants were instructed when to engage in volitional movement and when to rest by visual cues on a projection screen in the scanner.

\section{Transcranial magnetic stimulation (TMS)}

On a separate day, TMS motor mapping was conducted using a MagPro X100 stimulator unit with C-B60 coil (a figure-eight coil having a $35 \mathrm{~mm}$ inner diameter, $75 \mathrm{~mm}$ outer diameter, $11 \mathrm{~mm}$ winding height, and two layers of five windings for each wing of the coil; MagVenture, Inc., Atlanta, Georgia USA) with a neuronavigation unit (Visor1, ANT, Netherlands). A $5 \times 5$ grid ( $1 \mathrm{~cm}$ spacing) was generated and centered on the empirically identified motor hotspot using custom Matlab scripts. At each grid point, single biphasic TMS pulses were delivered at an intensity of $120 \%$ resting motor threshold (RMT) of the first dorsal interosseous (FDI) muscle. The RMT was determined as the stimulator output that resulted in 5 out of 10 MEPs of at least $50 \mu \mathrm{V}$ peak to peak. Stimulation at each grid point was performed using two different coil orientations ( $45^{\circ}$ and $90^{\circ}$ to midline) during the same recording session. The current direction in the brain induced by the biphasic TMS pulse was AP-PA (first phase-second phase) for the $45^{\circ}$ orientation and ML-LM for the $90^{\circ}$ orientation. The order of orientation was counter-balanced across subjects. Coil position and orientation were recorded using the neuronavigation system and transformed to the coordinate system of the head models.

Motor evoked potentials were recorded using a Biometrics Ltd. (Ladysmith, Virginia, USA) K800 amplifier and SX230 EMG sensors ( $1 \mathrm{~cm}$ diameter, $2 \mathrm{~cm}$ spacing) placed over the longitudinal axis of the muscle belly of first dorsal interosseous (FDI). Data were acquired at $2 \mathrm{kHz}$ using a Digidata 1440A (Molecular Devices LLC, California, 
USA), viewed using Clampex 10.3 software (Molecular Devices LLC, California, USA), and stored on a computer for later analysis. Average MEPs were the result of 10 consecutive single biphasic TMS pulses delivered from a particular coil orientation $\left(45^{\circ}\right.$ or $\left.90^{\circ}\right)$ every $1-3 \mathrm{~s}$ at each grid position. A pulse was delivered only if the coil center was positioned $<1 \mathrm{~mm}$ from the target grid point as assessed by the neuronavigation system (Visor1, ANT, Netherlands). MEP amplitudes and latencies were computed using custom Matlab scripts. MEP maps were generated for each coil orientation from the averaged MEP amplitudes obtained in response to TMS pulses delivered at each grid location.

\section{Finite element models}

For each subject we constructed an individualized FEM model of the head based on their structural MRI and DTI image data. These FEM models were built using SimNibs as previously described (www. simnibs.org; Windhoff et al., 2013). Briefly, FEM models consisted of around 1.7 million tetrahedra. Mesh resolution was selectively enhanced in GM and WM regions with an average tetrahedron volume of $1 \mathrm{~mm}^{3}$. Electrical conductivities were assigned to different tissue types as previously described (Thielscher et al., 2011) where $\sigma_{\text {skin }}=$ $0.465 \mathrm{~S} / \mathrm{m}, \sigma_{\text {skull }}=0.010 \mathrm{~S} / \mathrm{m}, \sigma_{\mathrm{CSF}}=1.654 \mathrm{~S} / \mathrm{m}, \sigma_{\mathrm{GM}}=0.276 \mathrm{~S} / \mathrm{m}$, and $\sigma_{\mathrm{WM}}=0.126 \mathrm{~S} / \mathrm{m}$. Anisotropic conductivity information derived from the DTI data were included using a volume normalized mapping approach as described in Opitz et al. (2011). The vector potential of the TMS coil was calculated by approximating it with small magnetic dipoles, which were placed such that they cover the area of the coil as previously described (Thielscher and Kammer, 2004). For each TMS coil position and orientation studied, the electric field generated by TMS was simulated for all subjects.

\section{Data analysis}

Functional images were analyzed using FSL Feat (www.fmrib.ox.ac. uk/fsl; Smith et al., 2004) and coregistration between EPI and structural images was performed using FSL Flirt (Jenkinson et al., 2002) and Afni align_epi_anat (Saad et al., 2009).

For each subject and coil orientation, the traditional center of gravity ( $\mathrm{CoG}$ ) was computed and projected on the brain surface (Diekhoff et al., 2011) and the Euclidian distances between the $45^{\circ}$ and $90^{\circ}$ coil orientation were calculated. Similar to the calculation of the TMS CoG position an electric field "Center of Gravity" $\left(E_{\operatorname{cog}}\right)$ was calculated by taking an MEP amplitude weighted sum of the electric field strengths for each node in the mesh over the 25 grid positions, where $E_{\operatorname{cog}}=\sum_{i} \frac{M E P_{i}}{M E P_{\text {total }}}\left|\vec{E}_{i}\right|$, with $M E P_{i}$ the mean MEP amplitude of position $i, M E P_{\text {total }}$ the sum of all MEPs and $\left|\vec{E}_{i}\right|$ the absolute electric field strength for position $i$ at the respective node. A combined electric field CoG was computed by multiplying the electric field CoGs of the $45^{\circ}$ and $90^{\circ}$ coil orientations. The rationale behind this was that the functional relevant regions for TMS yield high field strengths in both orientations and that those areas, which are only co-activated have high field strengths only for one orientation and not the other.

To examine the effects of coil orientation on MEPs, differences of MEP latencies and amplitudes obtained in response to TMS pulses delivered using the same coil position (grid location) for $45^{\circ}$ and $90^{\circ}$ orientations were calculated. To investigate the influence of the local gyral anatomy, the curvedness of the individual hand knob region was estimated by taking the median over the curvedness of the triangles approximating its shape. The hand knob region was determined by transforming a mask drawn in MNI space back to the individual subject space. Curvedness was calculated as $C=\sqrt{\frac{k_{1}^{2}+k_{2}^{2}}{2}}$, with $k_{1}$ and $k_{2}$ displaying the principal curvatures (Pienaar et al., 2008).
The perpendicular component of the electric field $\left(E_{\perp}\right)$ at the cerebrospinal fluid-gray matter (CSF-GM) interface in M1 was calculated as $E_{\perp}=\vec{E} \cdot \vec{n}$, where $\vec{E}$ was the electric field and $\vec{n}$ was the normal vector of the triangle. In addition, the tangential component of the electric field $\left(E_{\|}\right)$was calculated where $E_{\|}=\left|\vec{E}-\vec{E}_{\perp}\right|$. Only regions having a BOLD contrast $z$-score for movement versus rest $>2.3$ during voluntary movement of the index finger were taken into account. Similarly the component of the electric field in direction of the first eigenvector of the diffusion tensor at the gray matter-white matter (GM-WM) interface in primary motor cortex (M1) was computed as $E_{D_{\|}}=\overrightarrow{\mathrm{E}} \cdot \vec{V}_{1}$, where $\vec{E}$ was the electric field and $\vec{V}_{1}$ was the first eigenvector of the diffusion tensor. Furthermore, the perpendicular component was computed as $E_{D_{\perp}}=\left|\vec{E}-\vec{E}_{D_{\|}}\right|$.

The relationship between the electric field strength in M1 and MEP amplitude was tested with a linear regression model: $M E P_{i} \sim E_{\perp i}+E_{\| i}+E_{D_{\|} i}+E_{D_{\perp} i}$, with the dependent variable $M E P_{i}$ set to the mean MEP amplitude of grid point $i$, the explanatory variable $E_{\perp i}$ is equal to the mean perpendicular component of the electric field in $\mathrm{M} 1, E_{\| i}$ the mean tangential component of the electric field in M1, $E_{D_{\|} i}$, is the mean electric field in principal diffusion direction in $\mathrm{M} 1$ and $E_{D_{\perp} i}$ the mean electric field component perpendicular to the principal diffusion direction in M1 for grid point $i$, respectively. Only grid points with a reliable mean MEP value $>50 \mu \mathrm{V}$ were taken into account. Regression models were calculated for each subject and coil position separately. To analyze the effect of coil orientation on MEP amplitude and latency, separate two-tailed t-tests were used. The MEP hot spots were empirically derived as the grid location having the highest mean MEP amplitude in response to TMS. The location of this hot spot differed for subjects across coil angles. In some cases, the MEP amplitudes and latencies obtained at these hot spots were compared between subjects. To further study the influence of coil orientation on an individual basis, MEP amplitudes were collapsed across the grid and compared within subjects between coil orientations using paired t-tests. All data shown are mean \pm standard deviation and p-values less than 0.05 were considered significant.

\section{Results}

\section{Functional imaging}

We examined fMRI BOLD contrast maps (finger movement $>$ rest) for volitional right index finger abduction. All subjects exhibited robust BOLD activations at the crown of the left precentral gyrus $(z>2.3)$ that extended deep along the anterior bank of the central sulcus (Fig. 1A) consistent with previous observations (Porro et al., 1996). For each subject, BOLD volumes of the precentral gyrus that exceeded statistical threshold $(z>2.3)$ were used as regions of interest for examining electric fields induced by TMS.

\section{Comparison of simulation results obtained with finite element and projection models}

We used computer simulations to examine how tilting or rotating the TMS coil would influence the spatial distribution of brain targets and contrasted the results obtained using projection-based approaches with those from FEM models. In these simulations we varied the coil tilt angle by changing its elevation from $-30^{\circ}$ to $+30^{\circ}$ in $10^{\circ}$ increments at a single grid location. Similarly we modeled the influence of TMS coil orientation by changing its angle with respect to the midline in steps of $45^{\circ}$ from $0^{\circ}$ to $135^{\circ}$. For each TMS coil condition modeled, we projected the center point of the coil onto the cortical surface using conventional targeting methods (Fig. 1B). We also simulated the electric field using the FEM for each TMS coil condition (Fig. 2). From the simulation data, we calculated the Euclidian distances from the projected 


\section{A BOLD contrast in response to volitional FDI activation}

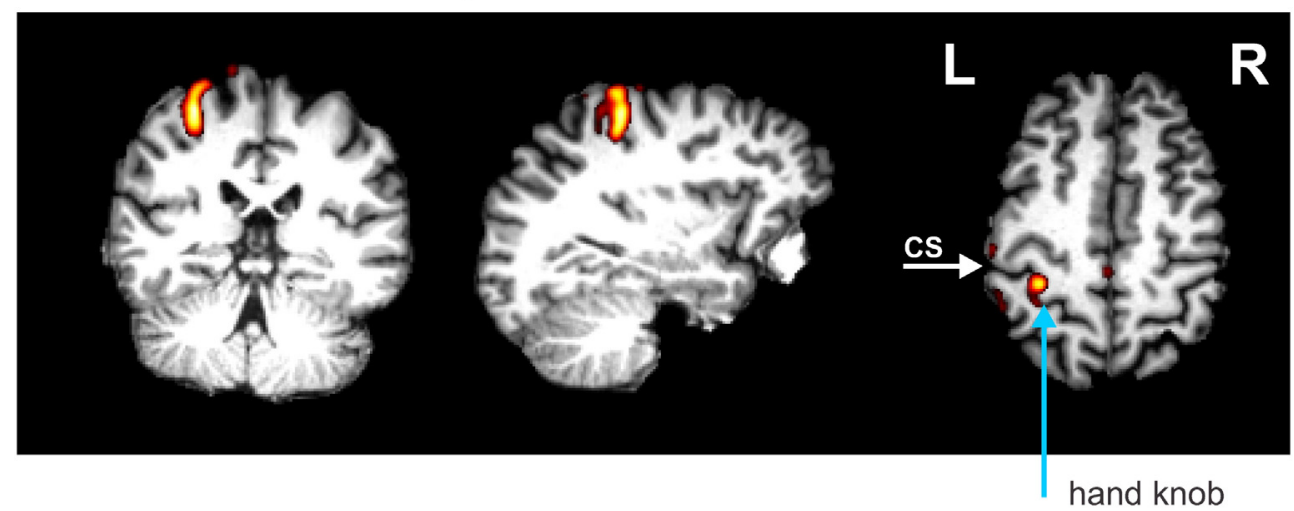

B
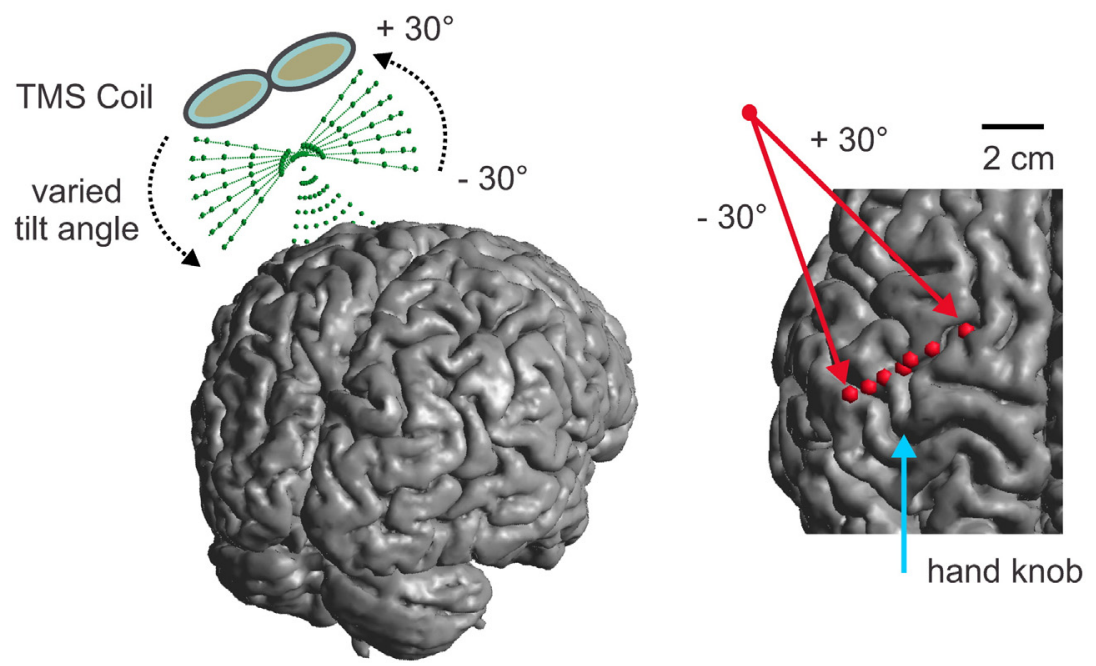

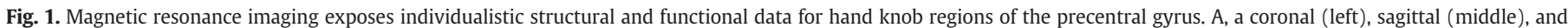

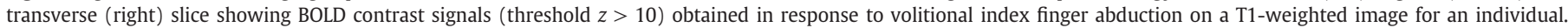

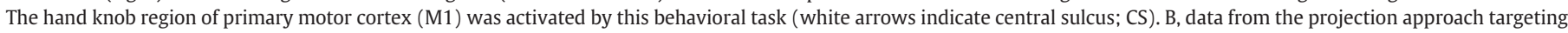

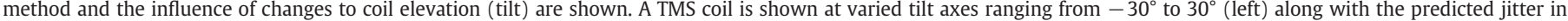
cortical stimulation targets obtained by projecting the coil centers on the cortical surface (right).

points on the cortical surface to the projected point of the $0^{\circ}$ coil tilt angle, as well as relative changes in the absolute electric field strength.

We observed displacements ranging from 5 to $20 \mathrm{~mm}$ between the projection point of the CoG on the cortex and the center point of the coil on the scalp when changes to the elevation (tilt) of TMS coils were modeled (Fig. 1B). Rotation of the coil orientation angle also produced small fluctuations in the distribution of the magnetic vector potential on the scalp (Supplementary Fig. 1). Using projection-based approaches we observed that spatial shifts with respect to the brain areas affected by TMS were more prominent when coil elevation was varied compared to rotation of the coil (Fig. 1B and Supplementary Fig. 1). Interestingly, FEM models predicted somewhat of the opposite where changes to TMS coil rotation would produce more robust shifts of the electric field compared to those elicited by changing the coil elevation. Here changes to the elevation of the coil produced less than $2 \%$ of an effect on the spatial distribution of the relative electric field (Fig. 2A), whereas rotation of the TMS coil elicited shifts in distribution of the relative electric field by $23.7 \pm 9.6 \%$ per $45^{\circ}$ change (Fig. $2 \mathrm{~B}$ ).

The above comparisons show that electric field distribution is strongly influenced by coil rotation while the projected point of the coil center remains relatively stable since the coil axis does not change with rotation. Thus, projection approaches do not appear sensitive enough to capture differences in the regions of brain affected by changes to the orientation angle of the TMS coil (Supplementary Fig. 1B). To further examine this issue, we analyzed neurophysiological data obtained while recording changes in motor evoked potentials (MEPs) in response to TMS pulses delivered at varied, MR-targeted spatial positions using two different coil orientations in volunteer subjects.

Motor evoked potential variability stemming from stimulator coil position and orientation

Although several observations indicate otherwise (Balslev et al., 2007; Fox et al., 2004; Opitz et al., 2011; Thielscher et al., 2011), it is generally accepted TMS coils should be positioned $45^{\circ}$ relative to the midline to achieve optimal stimulation of motor cortex (Brasil-Neto et al., 1992; Mills et al., 1992). We stimulated 25 discrete locations over the cortex using a $5 \times 5$ ( $1 \mathrm{~cm}$ spacing) grid centered on the FDI hotspot using two different coil orientations ( $45^{\circ}$ and $90^{\circ}$ to the midline; Fig. 3 ).

There were no significant effects of coil orientation angle on the mean latencies of FDI motor evoked potentials (MEPs) across individuals $\left(\mathrm{N}=5 ; \mathrm{t}(4)=1.54, \mathrm{p}=0.20 ; 45^{\circ}\right.$ MEP latency $=32.40 \pm 0.77 \mathrm{~ms}$, $90^{\circ}$ MEP latency $=32.01 \pm 1.37 \mathrm{~ms}$; Fig. 4A). There was not a significant difference of the mean MEP amplitudes between subjects in response to the $45^{\circ}$ and $90^{\circ}$ coil orientations $(\mathrm{t}(4)=0.92, \mathrm{p}=0.41$; $45^{\circ}$ FDI MEP $=897.80 \pm 257.70 \mu \mathrm{V}$ versus $90^{\circ}$ FDI MEP $=805.40 \pm$ $435.31 \mu \mathrm{V}$ ) likely due to a high degree of inter-individual variability. Within subject comparisons however showed that some individuals responded more robustly to one TMS coil orientation versus another. For example, some individuals exhibited larger MEP amplitudes in 


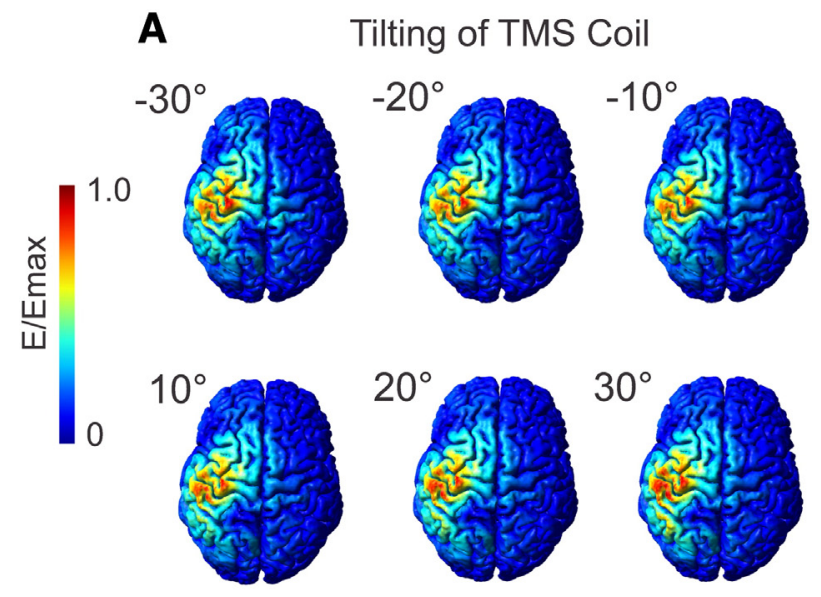

B Rotation of TMS Coil

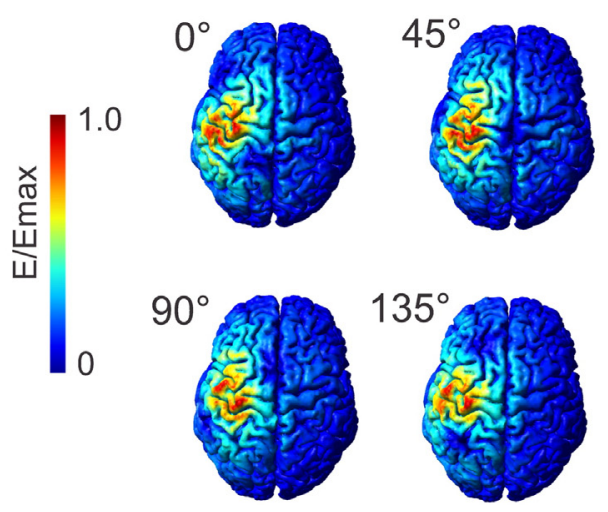

Fig. 2. The TMS-induced electric field in the brain is more prominently affected by changes in coil rotation than coil tilt. A, finite element models reveal that tilting the coil in $10^{\circ}$ steps produces a slight anterior-posterior shift in the spatial extent in the electric field as shown. B, modeling rotations of the TMS coil in $45^{\circ}$ increments indicated more robust changes in the location of the peak electric field, as well as to its spatial distribution compared to changes in coil elevation. Gyri with high field strengths are determined through the direction of the current flow with respect to the individual gyrification.

response to TMS pulses delivered using a $90^{\circ}$ coil orientation compared to a $45^{\circ}$ coil orientation while other volunteers displayed the opposite (Fig. 4A). These differences were significant for all individuals (paired $t$-test, $\mathrm{p}<0.01$ ) where two subjects (Subjects 4 and 5 ) exhibited significantly larger MEP amplitudes across the stimulation grid in response to a $90^{\circ}$ coil angle and three subjects (Subjects $1-3$ ) had significantly larger MEP amplitudes in response to a $45^{\circ}$ coil angle (Figs. $4 \mathrm{~A}$ and 5). These differences were unlikely due to a spatial jitter of coil orientation or position since the mean coil angle typically varied $<0.5^{\circ}$ while its mean position varied on average $<1 \mathrm{~mm}$ from trial-to-trial (Fig. 4B). Since FEM simulations have recently indicated that gyral curvature influences the distribution of the electric field induced by a TMS pulse (Opitz et al., 2011), we next aimed to determine how the shape of the hand knob region of M1 influences MEP variability.

\section{Gyral curvature influences transcranial magnetic stimulation efficacy}

The shape and curvature of the hand knob region of M1 vary across individuals. Approximately $90 \%$ of individuals have a hand knob shape described by an inverted omega (" $\Omega$ "), while the remaining $10 \%$ of individuals have an epsilon-shaped (" $\varepsilon$ ") hand knob (Caulo et al., 2007; Yousry et al., 1997). We thus questioned how the shape of the hand knob with respect to TMS coil orientation angle influences MEP amplitudes observed across the stimulus grid. We observed that subject's having a hand knob shaped like an inverted omega responded preferentially to a $45^{\circ}$ coil angle while one subject having a hand knob shaped like an epsilon responded preferentially to a $90^{\circ}$ coil angle (Fig. 5).

In order to make a more quantitative assessment of the observation described above, we calculated the curvature of the hand region of M1 for subjects (Fig. 6A). We then compared the median curvature of individual subject's hand knobs against the difference of their MEP amplitudes evoked at $45^{\circ}$ and $90^{\circ}$ coil angles across the stimulus grid. We observed an inverted-u-shaped relationship between hand knob curvature and coil orientation preference where subject's having either weakly curved $(<0.22)$ or strongly curved $(>0.25)$ hand knobs exhibited larger MEP amplitudes in response to a $90^{\circ}$ TMS coil orientation angle while subjects with median curvatures $>0.22$ and $<0.25$ exhibited larger FDI MEP amplitudes in response to TMS pulses delivered from a $45^{\circ}$ coil angle (Fig. $6 \mathrm{~B}$ ).

Finite element model simulations of electric fields correlate with physiological observations

Our simulations revealed that electric field distributions in GM and WM vary as a function of the orientation angle of the TMS coil and gyral curvature (Fig. 6C; Supplementary Figs. 2 and 3). Convolving the $E_{\mathrm{cog}}$ modeled for $45^{\circ}$ and $90^{\circ}$ coil angles clearly revealed M1 as the primary targeted area irrespective of coil orientation (Fig. 6D). The spatial distribution of the electric field itself however, changed quite dramatically as a function of TMS coil angle. Consistent with recent observations (Opitz et al., 2011; Thielscher et al., 2011), our models indicated that gyri oriented perpendicularly to electric current flow experience high electric field strengths. Our FEM models showed that gyri neighboring M1 experienced high field strengths if they were oriented perpendicularly to the direction of current flow generated by a particular TMS coil angle (Supplementary Figs. 2 and 3). These data illustrate that the direction of current flow with respect to gyral orientation is a key factor for determining the electric field strength generated by TMS pulses. Providing an initial physiological validation of FEM approaches to estimating electric fields elicited by TMS pulses, our modeling observations are in good agreement with our physiological results where FDI MEP amplitudes varied as a function of coil orientation angle (Figs. 4A and $5 \mathrm{~B}$ ) and hand knob curvature (Fig. 6B).

Our FEM results indicated that TMS pulses can induce a robust $E_{\operatorname{cog}}$ in gyral crowns, as well as deeper in the WM regions of brain tissue (Fig. 6D). These FEM data suggest that electric fields generated in response to TMS pulses might be able to activate different neuronal populations located in those areas of high field strength. Further, the results are consistent with fMRI BOLD signals observed in response to volitional index finger abduction (Figs. 7A, B). We found the mean perpendicular component of the simulated electric field generated in M1 to be closely related to MEP amplitudes observed in response to a TMS pulse (Fig. 8). A similar pattern emerged for the relationship between the electric field in principal diffusion direction of white matter and the MEP amplitudes recorded during our TMS motormapping studies (Fig. 8). While the field strength of the mean tangential component and the component perpendicular to the principal diffusion direction had higher electric field values, their relationship to the MEPs was similar compared to the other two components (Fig. 8). Finally, there were significant correlations for the regression of the MEP amplitudes against the four electric field components at M1 for each subject's preferred coil orientation in four out of five subjects $(S)$. The Pearson's $r$ and p-values for these correlations were as follows: $\mathrm{S} 1 \mathrm{r}=0.82, \mathrm{p}=0.0014$; $\mathrm{S} 2 \mathrm{r}=0.91, \mathrm{p}=$ $1.8 \times 10^{-5} ; \mathrm{S} 3 \mathrm{r}=0.70, \mathrm{p}=0.052 ; \mathrm{S} 4 \mathrm{r}=0.83, \mathrm{p}=3.7 \times 10^{-4}$; and $\mathrm{S} 5 \mathrm{r}=0.80, \mathrm{p}=0.0048$.

\section{Discussion}

Measuring MEPs elicited by TMS of the motor cortex represents one of the most commonly employed "biomarkers" for quantifying the effects of a variety of neuromodulation strategies on plasticity. 
A
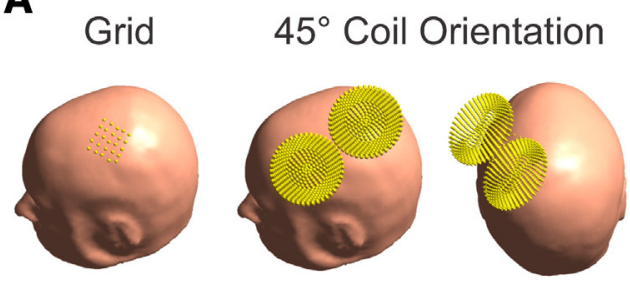

Grid
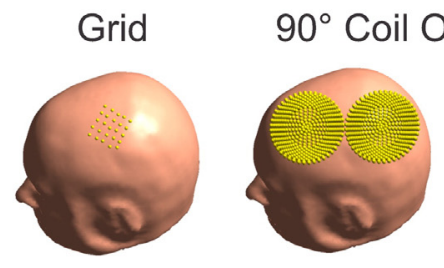
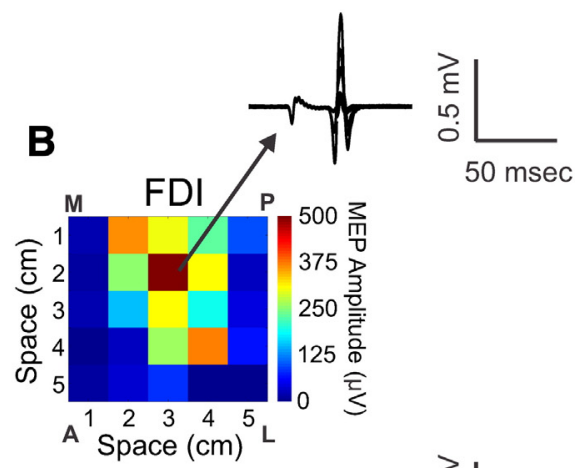

$50 \mathrm{msec}$

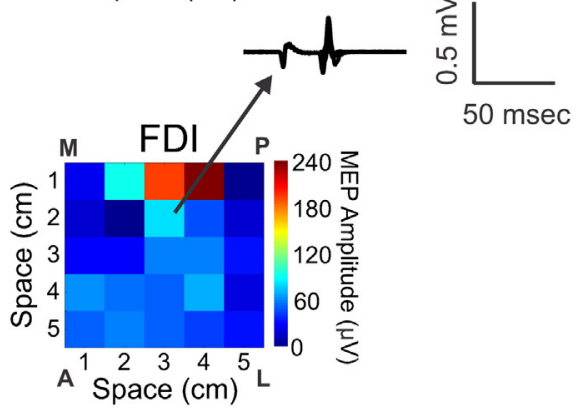

Fig. 3. Transcranial magnetic stimulation of the motor cortex using $45^{\circ}$ and $90^{\circ}$ coil orientation angles can be used to develop individual motor excitability maps. A, a $5 \times 5$ stimulation grid ( $1 \mathrm{~cm}$ spacing) is shown centered over the FDI hotspot on a head model for an individual (left). Two different views illustrate a model of the TMS coil positioned at $45^{\circ}$ (top) and $90^{\circ}$ (bottom) over the motor cortex. B, FDI motor excitability maps generated by stimulating 25 points across the grids shown in (A) using a $45^{\circ}$ (top) and $90^{\circ}$ (bottom) coil angle are illustrated for an individual. Medial $(\mathrm{M})$, posterior $(\mathrm{P})$, anterior $(\mathrm{A})$, and lateral $(\mathrm{L})$ anatomical orientations of the motor excitability map are indicated. Each pseudo-colored square illustrates the mean FDI motor evoked potential (MEP) amplitude obtained in response to 10 stimuli delivered using TMS pulses at $120 \%$ of the motor threshold over every point of the grid. For both coil orientations, individual MEP responses $(\mathrm{N}=10)$ are shown for the FDI hotspot identified using a conventional $45^{\circ}$ coil angle.

\section{A MEP response latencies ( $\mathrm{msec})$}

\section{Normalized MEP amplitudes}
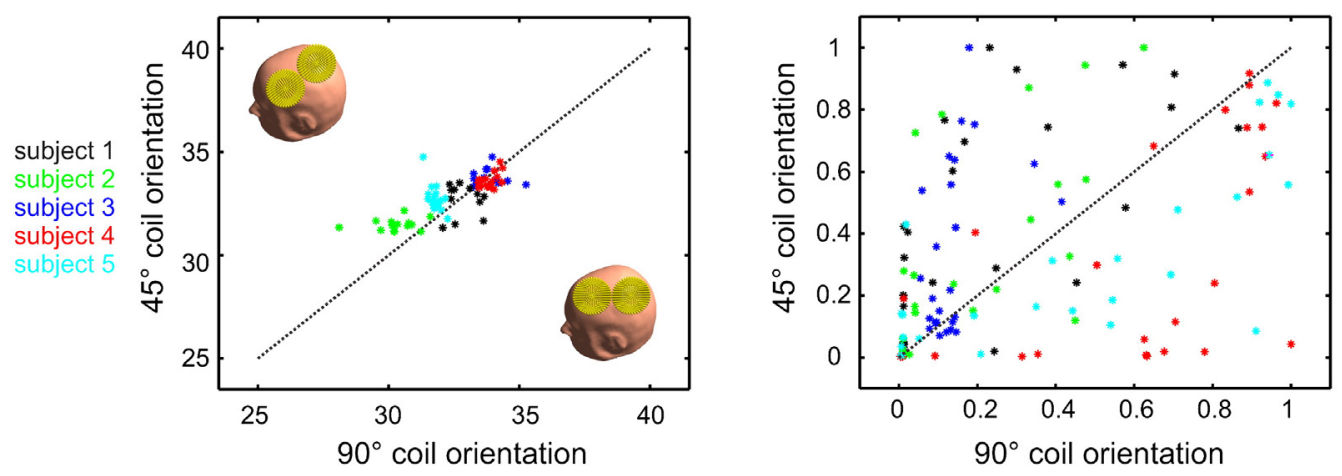

\section{B Coil jitter}

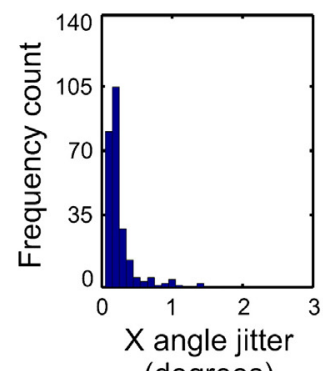

(degrees)

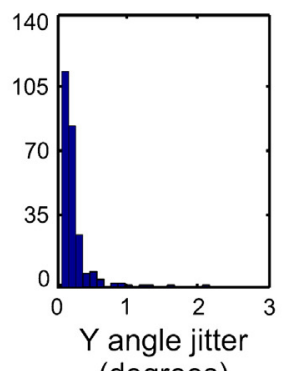

(degrees)

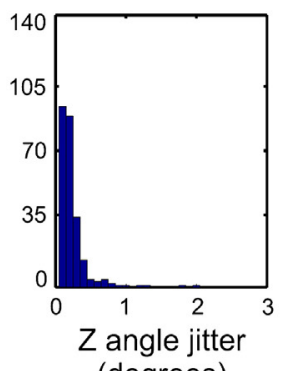

(degrees)

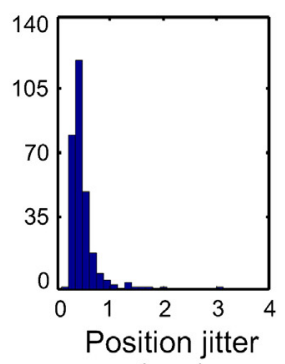

$(\mathrm{mm})$

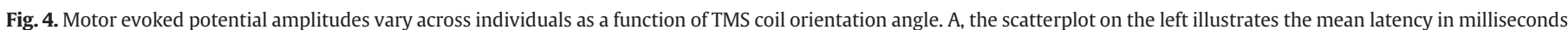

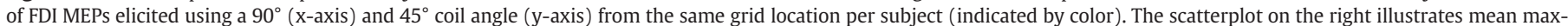

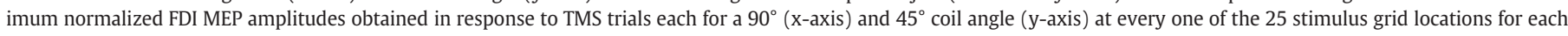

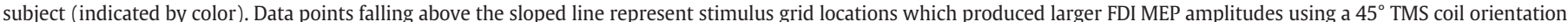

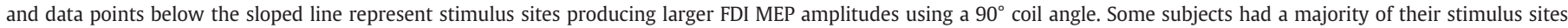

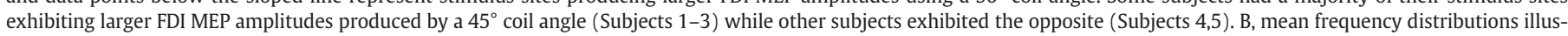
trating the spatial jitter of coil placement across all grid positions $(\mathrm{N}=25)$ and orientations $(\mathrm{N}=2)$ for all subjects $(\mathrm{N}=5)$. 
A Subject 2
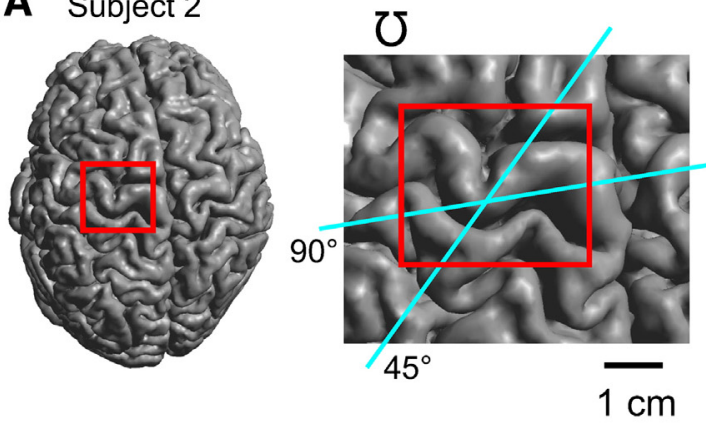

Subject 3
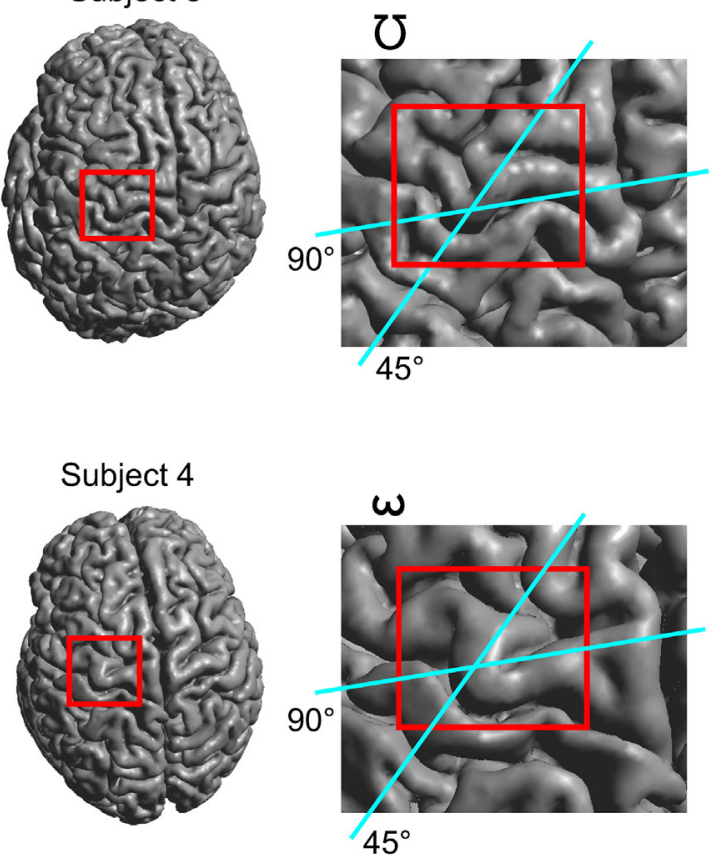

B

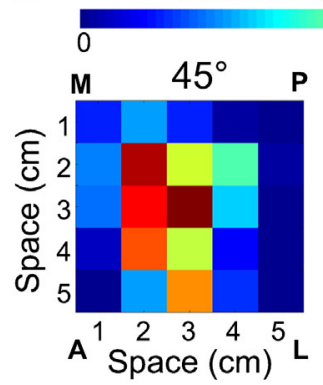

FDI MEP Amplitude $(\mu \mathrm{V})$

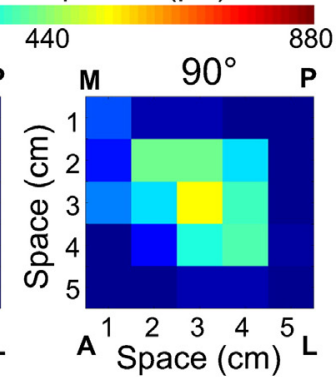

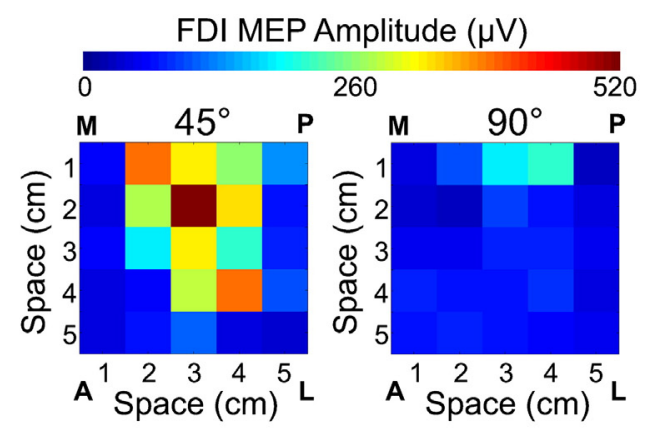

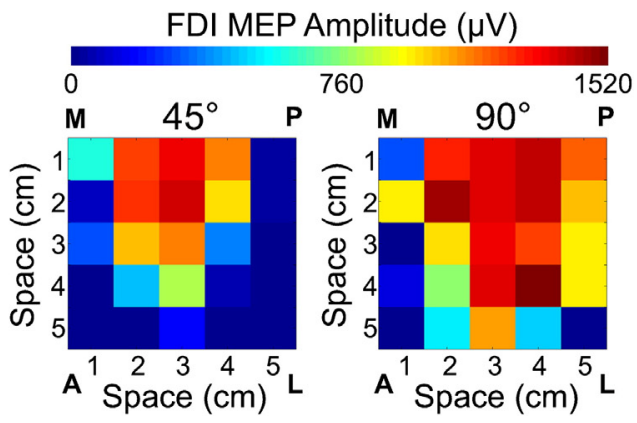

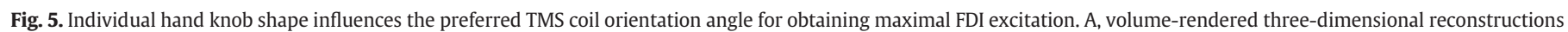

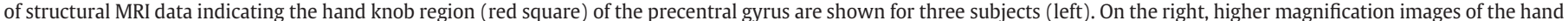

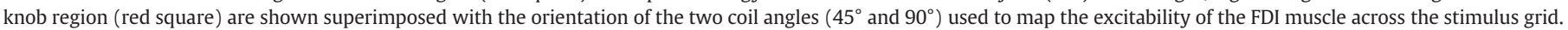

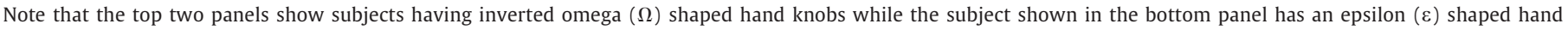

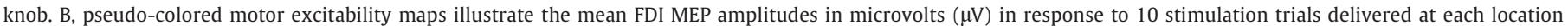

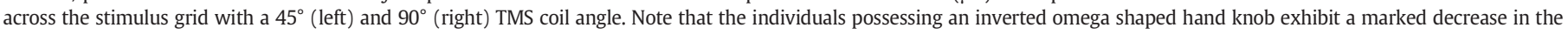
FDI MEP amplitudes across the grid for a $90^{\circ}$ versus a $45^{\circ}$ coil angle, while the individual possessing an epsilon shaped hand knob displayed the opposite relationship.

This basic approach and several other applications of TMS however continue to suffer from variable outcomes. Minimizing this variability can likely be achieved by increasing our understanding of how to more accurately, consistently, and reliably target brain circuits with TMS. Therefore we investigated the influence of inter-individual neuroanatomical characteristics like hand knob curvature and procedural variables like TMS coil orientation angle, which both affect physiological responses to TMS. We observed that traditional projection-based targeting methods do not sufficiently account for the above anatomical and procedural variables. Using FEM simulations of the TMS-induced electric field to more adequately account for anatomical and procedural variables, in the present study, we found that the strength of the modeled electric field in M1 significantly correlated with MEP amplitudes on an individual basis. These findings validate the use of FEM simulations as a more reliable approach to subject-specific TMS targeting compared to projection-based methods. Our observations further indicate the optimal coil orientation angle used during TMS studies or treatments can be predicted using FEM simulations and should be based upon an individual's specific gyral folding patterns and tissue conductivity anisotropy.
Spherical models predict electric field strengths at gyral crowns that remain spatially stable across varied TMS coil orientation angles. Conversely, FEM simulations indicate that electric field distributions experience prominent spatial shifts when the TMS coil orientation angle changes (Thielscher et al., 2011; Supplementary Figs. 2 and 3). Consistent with the predictions made by these FEM simulations, our physiological observations indicate that the efficacy of TMS depends mainly on coil angle (Fig. 5) with respect to the orientation and curvature of an individual's gyri (Fig. 6). Additionally, the strength and shape of the TMS-induced electric field are dependent on specific brain tissue electrical properties, which cannot be captured by spherical models or projection approaches. Thus compared to these conventional projection-based predictions, we conclude that FEM simulations of the electric field represent a more precise estimate of brain regions targeted by TMS. While these FEM-simulated electric field distributions do not predict discrete cellular points of stimulation, their more realistic estimation of the brain regions impacted should enable us to more confidently unravel the biophysical mechanisms of action underlying the ability of TMS to modulate brain circuit activity. 
A

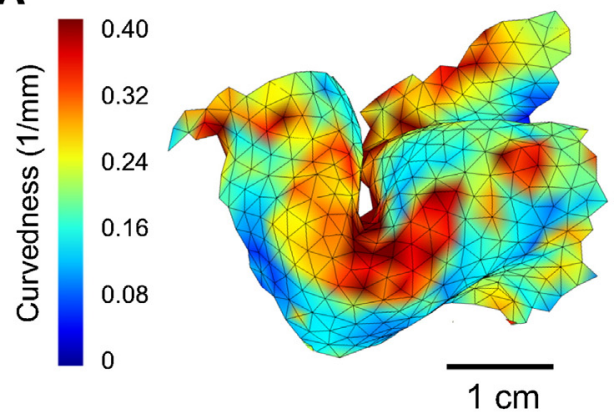

B

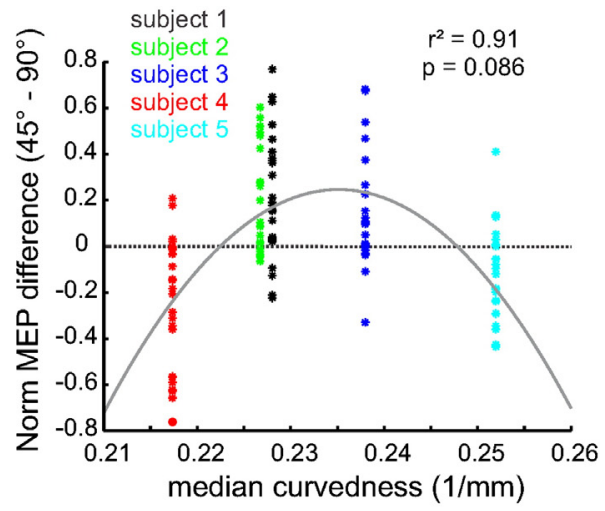

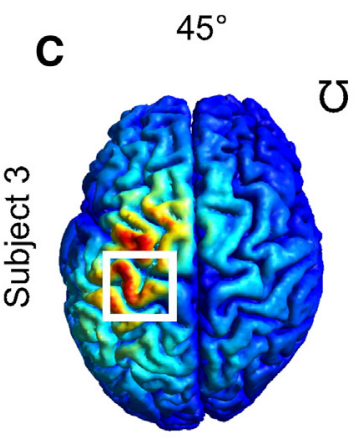
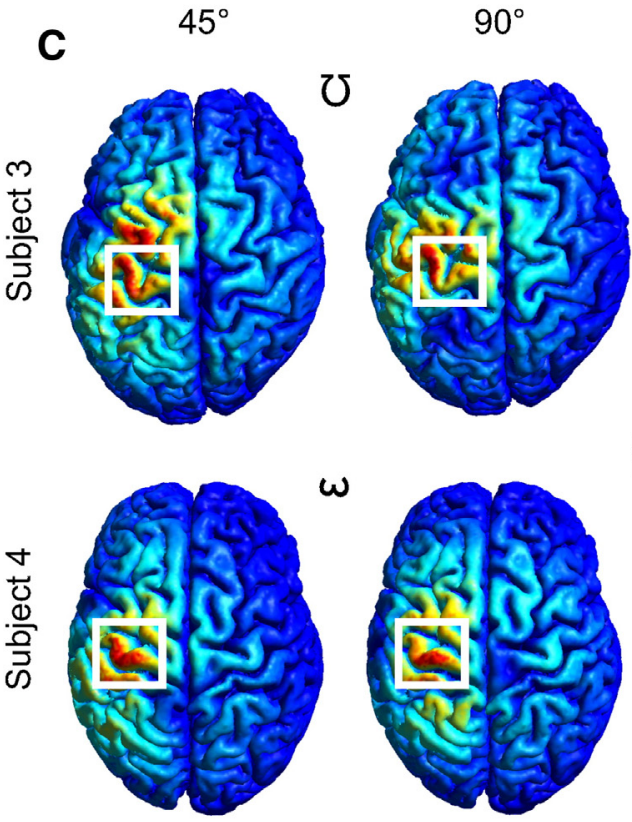

$\omega$

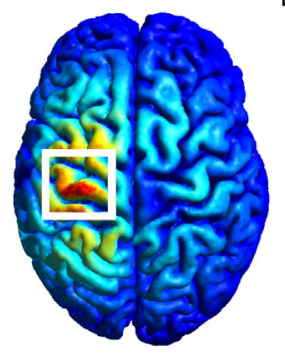

D
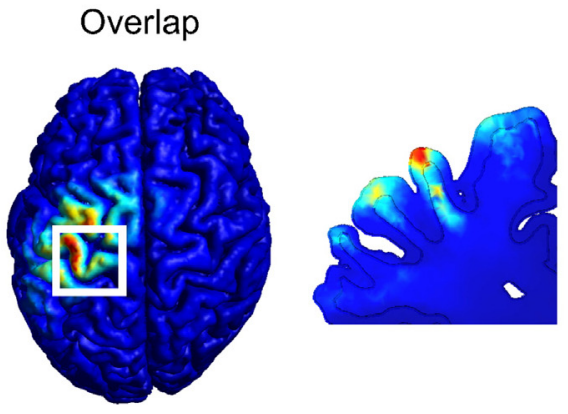

离

0.50

0.25
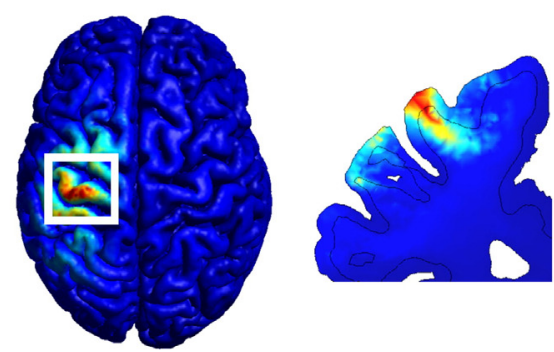

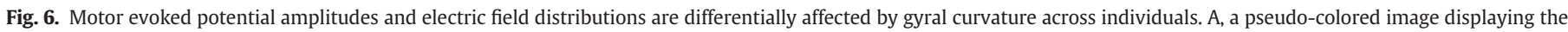

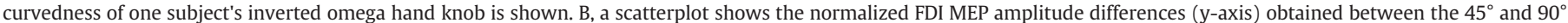

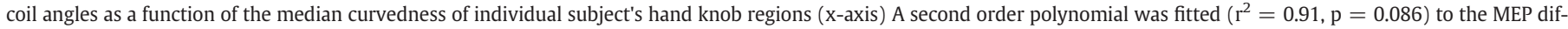

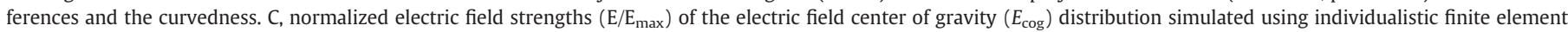

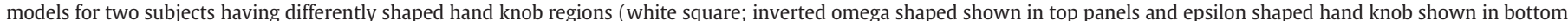

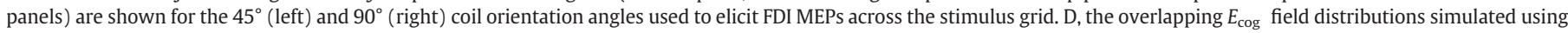

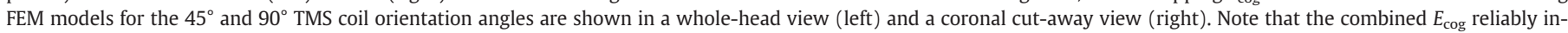
dicates M1 as the activated region. This is not a trivial effect as electric fields vary strongly over different coil positions (see Supplementary Figs. 2 and 3 ).

Possible limitations of our study are that while different conductivity domains are determined individually, fine scale differences in conductivity within an individual (for example, within GM or WM) are not presently taken into account. Such differences do not change our interpretations since it has been demonstrated that slight changes in conductivity between domains do not significantly alter the electric field distribution (Thielscher et al., 2011). Similarly, Opitz et al. (2011) recently demonstrated modeling results are stable for different conductivity mapping approaches. Gross alterations in tissue conductivity under pathological conditions like stroke however can induce distortions and alter the spatial distribution of the electric field induced by TMS (Wagner et al., 2006). In our study we used healthy participants, so potential consequences of pathologies are unlikely. Future studies may wish to consider the variability of intra-individual tissue conductivity when implementing TMS across individuals of different ages or disease states. An additional potential source of error in our study could be related to the placement and recording of TMS coil positions using the neuronavigation system. While the jitter at each coil position was generally $1 \mathrm{~mm}$ or less, the algorithms mapping these recorded positions to MRI coordinates have a limited resolution resulting in measurement inaccuracies of about 3-5 mm (Ruohonen and Karhu, 2010). It is unlikely that these measurement inaccuracies exceed the $1 \mathrm{~cm}$ grid spacing employed in our mapping strategy and thus we feel confident in our ability to reliably distinguish individual grid positions.

In the present study we chose to implement biphasic pulses since they are known to have lower thresholds for generating MEPs compared to monophasic pulses (Kammer et al., 2001). The physics of TMS falls in the quasistatic domain, so the spatial and temporal domains can be decoupled from one another. In our study we deliberately focused on the spatial distribution of the electric field which is the same for both mono- and biphasic pulses. With respect to the temporal domain there are several timescales which need to be considered. One relates to the mechanisms of action underlying the activation or suppression of neuronal populations by a single TMS pulse, which is briefly discussed below. Another relates to physiological plasticity and how repeated spaced TMS pulses affect neuronal excitability across time (milliseconds to hours). Based on observations by others regarding pulse parameters required to induce plasticity on 
A fMRI BOLD

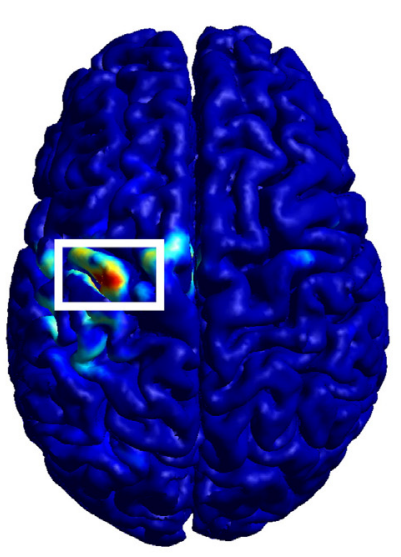

$E_{\text {cog }}$

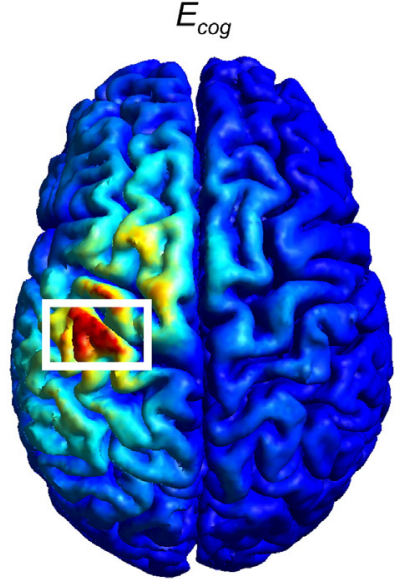

B
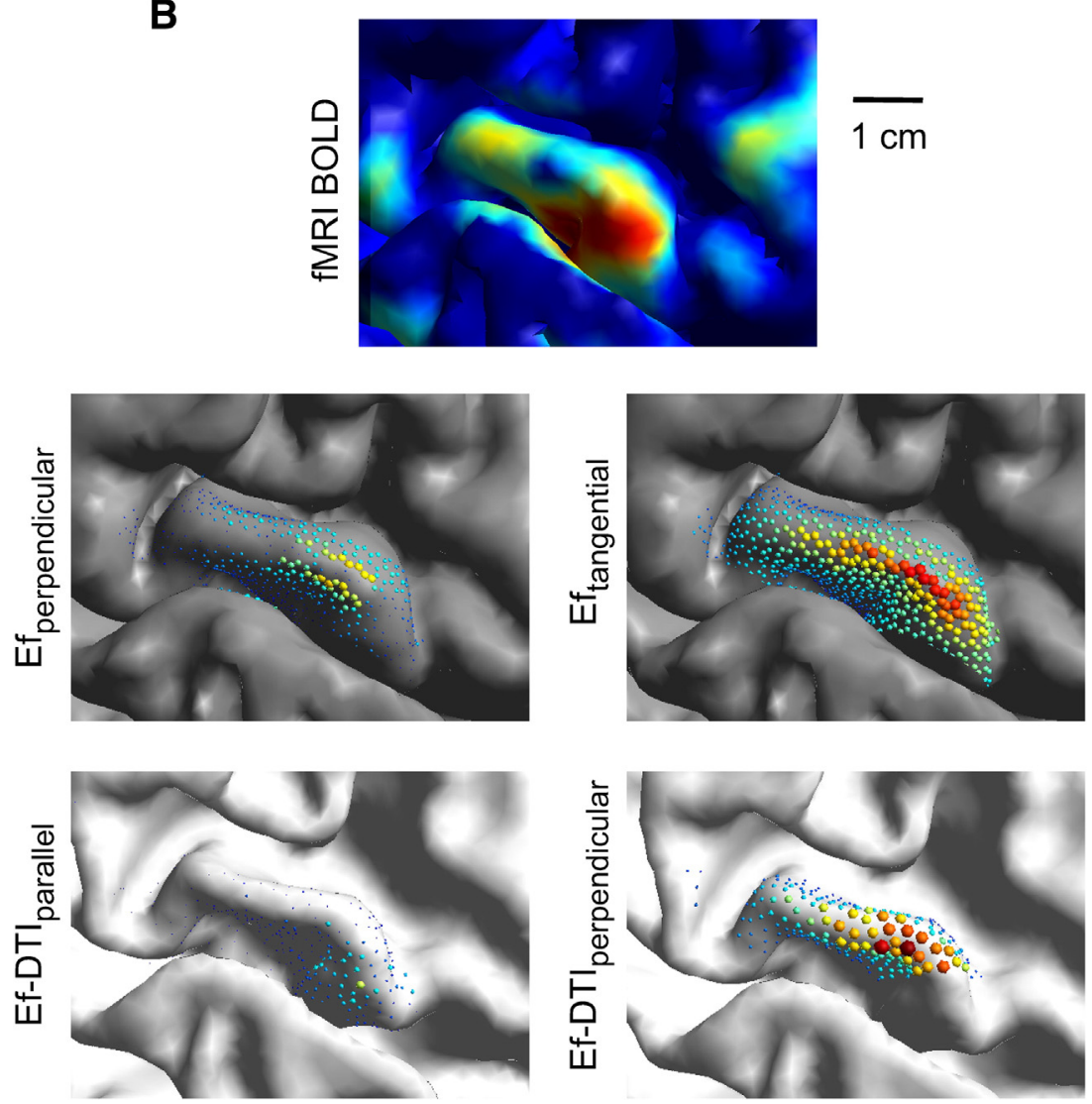

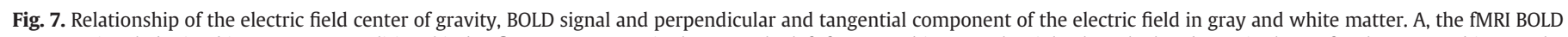

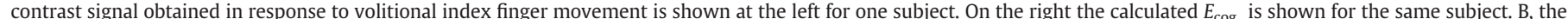

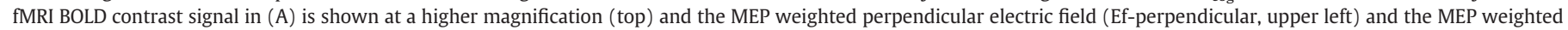

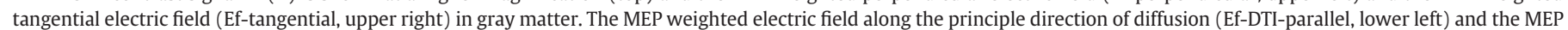

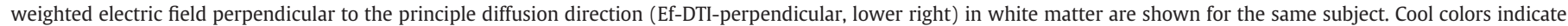

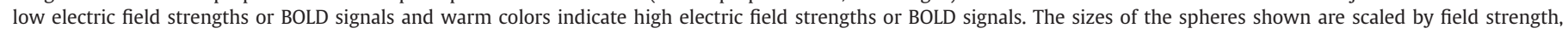
where larger spheres indicate higher electric field strengths. Electric fields in gray and white matter are scaled to the same maximum, respectively.

long timescales (minutes to hours; Ziemann et al., 2008), the low number of TMS pulses delivered to any one grid location was not likely to elicit robust long-lasting plasticity in our study. Perhaps a limitation to our study however, we used single pulse TMS having inter-trial intervals $<3 \mathrm{~s}$. Since TMS-evoked MEP amplitudes have been described to be affected to varying degrees when using an inter-trials $<10 \mathrm{~s}$ (Chen et al., 1997; Julkunen et al., 2012; Pascual-Leone et al., 1994), we cannot exclude the possibility that short-term plasticity may have influenced some of our observations. As fundamental properties of brain circuitry any such short-term physiological plasticity would likely be due to conventional synaptic mechanisms, such as receptor saturation, receptor trafficking, changes in channel gating kinetics, synaptic vesicle pool depletion, and dynamic regulation of neurotransmitter release probability, which have all been well-studied at corticocortical and corticothalamic synapses between pyramidal neurons, interneurons, and thalamic relay neurons (Sanes and Donoghue, 2000; Thomson, 2003; Zucker and Regehr, 2002). It is nearly impossible to determine which, if any, of these aforementioned mechanisms may have contributed to the trial-to-trial MEP variability we observed since it is not known with any certainty to what degree specific neuronal 


\section{A}

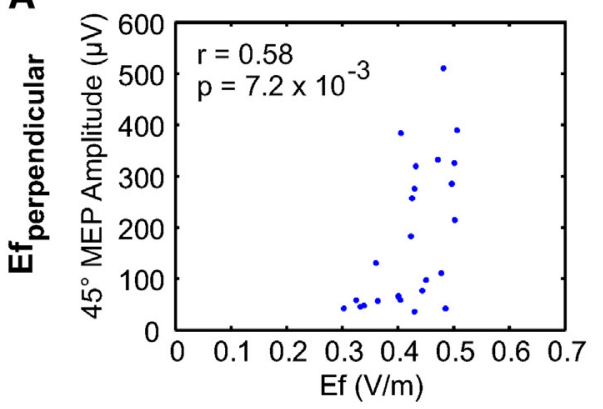

B

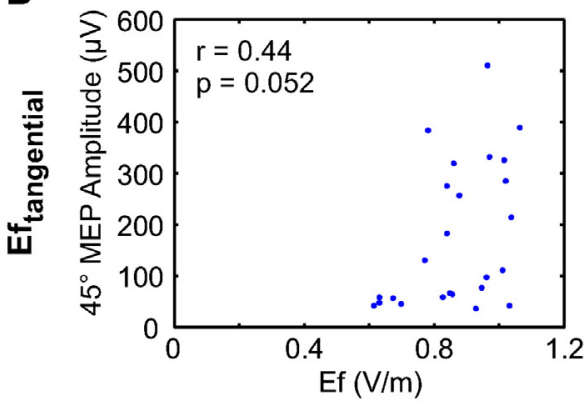

C

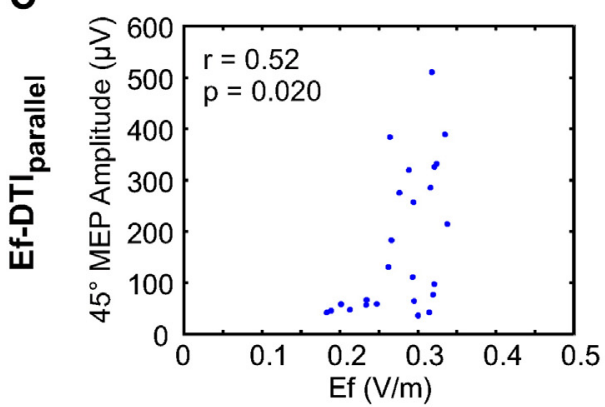

D

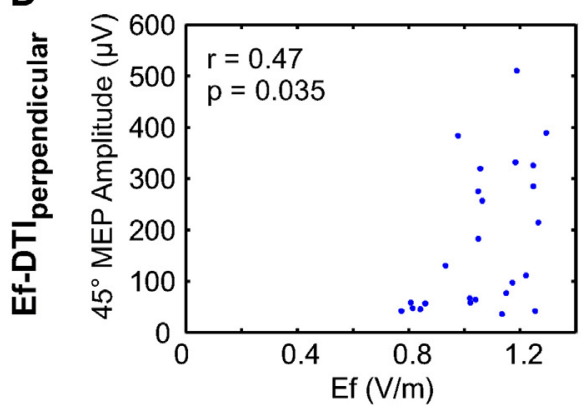

S4
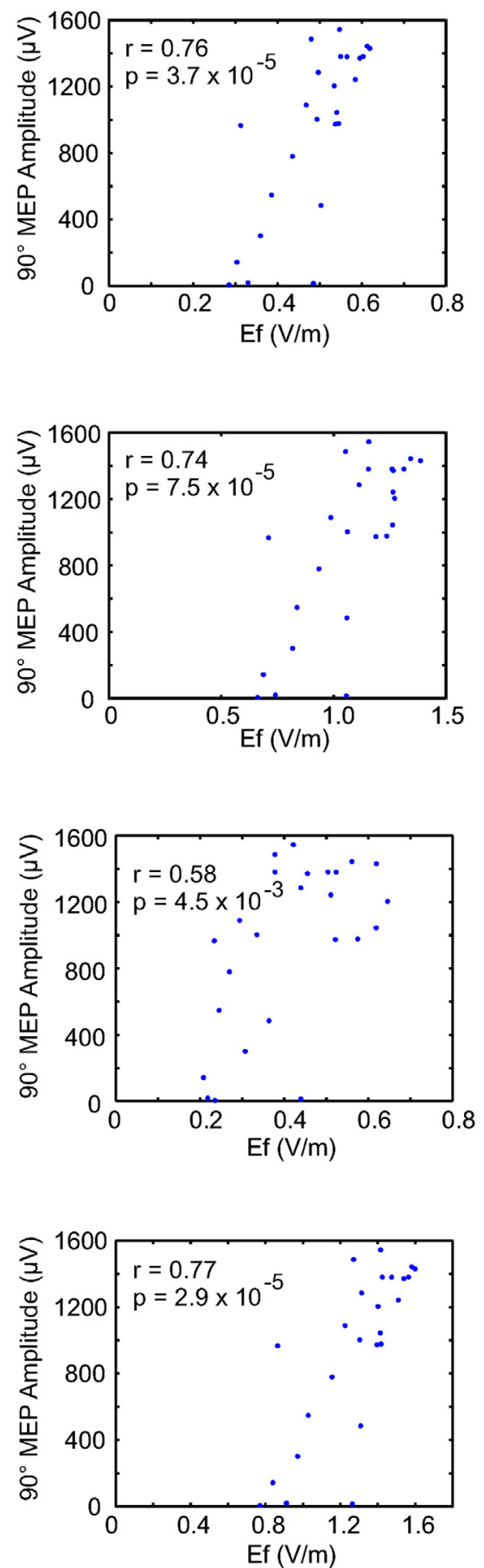

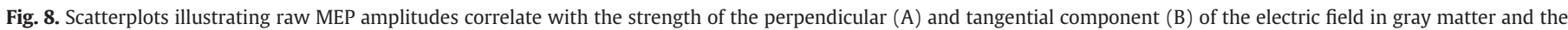

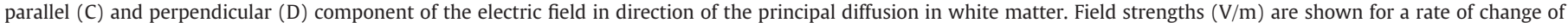
current flow in the coil of $1 \mathrm{~A} / \mu \mathrm{s}$.

elements are affected by the electric field induced by TMS. Future studies should however begin driving towards using high-resolution modeling to investigate how different time components of electric fields mediate physiological outcomes (including plasticity) induced by TMS across different timescales. This is a difficult but important and seemingly tractable problem if simulations can include faithful models of neuronal and synaptic populations which react differently to time varying pulse shapes and sequences. Such studies should shed light on the temporal behavior of TMS-induced electric fields while more accurately detailing the mechanisms of action across different embodiments of TMS.
One of the main objectives of the present study was to compare and contrast neurophysiological observations with the results from FEM simulations of TMS-induced electric fields. Comparisons of TMS CoG with the fMRI BOLD CoG have been recently reported (Diekhoff et al., 2011). Using similar approaches we found fMRI BOLD signals in response to voluntary index finger movement to be localized to primary motor and, of course, premotor and supplementary motor cortex. Comparing the fMRI BOLD CoG with the TMS-induced electric field center of gravity $\left(E_{\mathrm{cog}}\right)$, we found that the TMS-induced electric fields were concentrated on the primary motor cortex, as well as surrounding gyri during motormapping. Further, we found the normal component of 
the modeled electric field in these regions to be correlated with the amplitudes of TMS-elicited MEPs (Fig. 8A). An underlying physiological explanation might be that a current flowing perpendicular to a gyrus is optimally oriented to directly activate pyramidal neurons, which are mainly oriented horizontally in the sulcal wall. This interpretation is further supported by a correlation of the electric field strength along the principal diffusion direction of white matter and the MEP amplitudes (Fig. 8C), since the principal diffusion direction estimates a first-order approximation of the direction of the axons and high field strengths in this direction is a prerequisite for eliciting action potentials (Roth and Basser, 1990). On the other hand, the tangential component of the electric field at the GM/CSF interface also correlated with the MEP amplitudes (Fig. 8B). Perhaps best explained by a high intercorrelation with the tangential electric field at the GM/CSF interface, the component of the electric field perpendicular to the principal diffusion direction was similarly correlated with MEP amplitudes (Fig. 8D). At the top of the gyrus (GM/CSF interface), the tangential electric field is the predominant component and more directly supports hypotheses regarding the indirect trans-synaptic activation of pyramidal tract neurons via interneuron stimulation by TMS (Di Lazzaro and Ziemann, 2013). Yet from our observations we cannot reliably conclude a predominant site of action for TMS in evoking MEPs. Our results rather illustrate that the TMS-induced electric field magnitude explains approximately two-thirds of the MEP variability obtained across motor maps without specifying a particular site of action. Moreover, different mechanisms of action underlying the effects of TMS are still being debated (Salvador et al., 2011). By expanding combined observations made during physiological studies with individualized FEM simulation results however, we anticipate that advances in deciphering the mechanisms and sites of TMS action can be made in the near future.

Understanding the biophysics underlying TMS remains a difficult challenge and many factors determine how brain circuits are affected. Compared to traditional projection-based methods, we have shown that individualized computational physiology approaches employing FEM simulations of electric fields can better account some of the variables influencing physiological responses to TMS. By streamlining computations and processes for visualizing electric fields estimated by FEM models, the potential of TMS in research, diagnostics, and therapeutics should improve. For example, FEM simulations will enable the development of specific approaches intended to maximize the effects of TMS on targeted brain circuits in individuals. In conclusion our observations show that such personalized FEM models for targeting TMS are justified given the unique features of our individual brains.

Supplementary data to this article can be found online at http:// dx.doi.org/10.1016/j.neuroimage.2013.04.067.

\section{Conflict of interest}

The authors have no financial conflicts of interest related to the research conducted in this study.

\section{Acknowledgments}

The authors would like to thank Cameron Craddock and Jonathan Lisinski from the VTCRI for their advice on the fMRI acquisition. Furthermore we gratefully acknowledge the help of Jaap van der Spek and Lars Matthäus from ANT during integrating the Visor neuronavigation system with the FEM software. Finally we want to thank Mirko Windhoff from UBS Zürich for technical advice on the FEM solver.

\section{References}

Balslev, D., Braet, W., McAllister, C., Miall, R.C., 2007. Inter-individual variability in optimal current direction for transcranial magnetic stimulation of the motor cortex. J. Neurosci. Methods 162, 309-313.
Brasil-Neto, J.P., McShane, L.M., Fuhr, P., Hallett, M., Cohen, L.G., 1992. Topographic mapping of the human motor cortex with magnetic stimulation: factors affecting accuracy and reproducibility. Electroencephalogr. Clin. Neurophysiol. 85, 9-16.

Caulo, M., Briganti, C., Mattei, P.A., Perfetti, B., Ferretti, A., Romani, G.L., Tartaro, A., Colosimo, C., 2007. New morphologic variants of the hand motor cortex as seen with MR imaging in a large study population. AJNR Am. J. Neuroradiol. 28, 1480-1485.

Chen, M., Mogul, D.J., 2010. Using increased structural detail of the cortex to improve the accuracy of modeling the effects of transcranial magnetic stimulation on neocortical activation. IEEE Trans. Biomed. Eng. 57, 1216-1226.

Chen, R., Classen, J., Gerloff, C., Celnik, P., Wassermann, E.M., Hallett, M., Cohen, L.G., 1997. Depression of motor cortex excitability by low-frequency transcranial magnetic stimulation. Neurology 48, 1398-1403.

Di Lazzaro, V., Ziemann, U., 2013. The contribution of transcranial magnetic stimulation in the functional evaluation of microcircuits in human motor cortex. Front. Neural Circuits 7, 18.

Diekhoff, S., Uludağ, K., Sparing, R., Tittgemeyer, M., Cavuşoğlu, M., von Cramon, D.Y., Grefkes, C., 2011. Functional localization in the human brain: gradient-echo, spin-echo, and arterial spin-labeling fMRI compared with neuronavigated TMS. Hum. Brain Mapp. 32, 341-357.

Fox, P.T., Narayana, S., Tandon, N., Sandoval, H., Fox, S.P., Kochunov, P., Lancaster, J.L., 2004. Column-based model of electric field excitation of cerebral cortex. Hum. Brain Mapp. 22, 1-14

Jenkinson, M., Bannister, P., Brady, M., Smith, S., 2002. Improved optimization for the robust and accurate linear registration and motion correction of brain images. Neuroimage 17, 825-841.

Julkunen, P., Saisanen, L., Hukkanen, T., Danner, N., Kononen, M., 2012. Does secondscale intertrial interval affect motor evoked potentials induced by single-pulse transcranial magnetic stimulation? Brain Stimul. 5, 526-532.

Kammer, T., Beck, S., Thielscher, A., Laubis-Herrmann, U., Topka, H., 2001. Motor thresholds in humans: a transcranial magnetic stimulation study comparing different pulse waveforms, current directions and stimulator types. Clin. Neurophysiol. 112, 250-258.

Lefaucheur, J.P., Andre-Obadia, N., Poulet, E., Devanne, H., Haffen, E., Londero, A., Cretin, B., Leroi, A.M., Radtchenko, A., Saba, G., Thai-Van, H., Litre, C.F., Vercueil, L., Bouhassira, D., Ayache, S.S., Farhat, W.H., Zouari, H.G., Mylius, V., Nicolier, M., Garcia-Larrea, L., 2011. French guidelines on the use of repetitive transcranial magnetic stimulation (rTMS): safety and therapeutic indications. Neurophysiol. Clin. 41, 221-295.

Mills, K.R., Boniface, S.J., Schubert, M., 1992. Magnetic brain stimulation with a double coil: the importance of coil orientation. Electroencephalogr. Clin. Neurophysiol. 85, $17-21$.

Opitz, A., Windhoff, M., Heidemann, R.M., Turner, R., Thielscher, A., 2011. How the brain tissue shapes the electric field induced by transcranial magnetic stimulation. Neuroimage 58, 849-859.

Padberg, F., George, M.S., 2009. Repetitive transcranial magnetic stimulation of the prefrontal cortex in depression. Exp. Neurol. 219, 2-13.

Pascual-Leone, A., Valls-Sole, J., Wassermann, E.M., Hallett, M., 1994. Responses to rapid-rate transcranial magnetic stimulation of the human motor cortex. Brain 117 (Pt 4), 847-858.

Pienaar, R., Fischl, B., Caviness, V., Makris, N., Grant, P.E., 2008. A methodology for analyzing curvature in the developing brain from preterm to adult. Int. J. Imaging Syst. Technol. 18, 42-68.

Porro, C.A., Francescato, M.P., Cettolo, V., Diamond, M.E., Baraldi, P., Zuiani, C., Bazzocchi, M., di Prampero, P.E., 1996. Primary motor and sensory cortex activation during motor performance and motor imagery: a functional magnetic resonance imaging study. J. Neurosci. 16, 7688-7698.

Roth, B.J., Basser, P.J., 1990. A model of the stimulation of a nerve fiber by electromagnetic induction. IEEE Trans. Biomed. Eng. 37, 588-597.

Ruohonen, J., Karhu, J., 2010. Navigated transcranial magnetic stimulation. Neurophysiol. Clin. 40, 7-17.

Rusjan, P.M., Barr, M.S., Farzan, F., Arenovich, T., Maller, J.J., Fitzgerald, P.B., Daskalakis, Z.J., 2010. Optimal transcranial magnetic stimulation coil placement for targeting the dorsolateral prefrontal cortex using novel magnetic resonance image-guided neuronavigation. Hum. Brain Mapp. 31, 1643-1652.

Saad, Z.S., Glen, D.R., Chen, G., Beauchamp, M.S., Desai, R., Cox, R.W., 2009. A new method for improving functional-to-structural MRI alignment using local Pearson correlation. Neuroimage 44, 839-848.

Sack, A.T., Cohen Kadosh, R., Schuhmann, T., Moerel, M., Walsh, V., Goebel, R., 2009. Optimizing functional accuracy of TMS in cognitive studies: a comparison of methods. J. Cogn. Neurosci. 21, 207-221.

Salinas, F.S., Lancaster, J.L., Fox, P.T., 2009. 3D modeling of the total electric field induced by transcranial magnetic stimulation using the boundary element method. Phys. Med. Biol. 54, 3631-3647.

Salminen-Vaparanta, N., Noreika, V., Revonsuo, A., Koivisto, M., Vanni, S., 2012. Is selective primary visual cortex stimulation achievable with TMS? Hum. Brain Mapp. 33, 652-665.

Salvador, R., Silva, S., Basser, P.J., Miranda, P.C., 2011. Determining which mechanisms lead to activation in the motor cortex: a modeling study of transcranial magnetic stimulation using realistic stimulus waveforms and sulcal geometry. Clin. Neurophysiol. $122,748-758$

Sanes, J.N., Donoghue, J.P., 2000. Plasticity and primary motor cortex. Annu. Rev. Neurosci. 23, 393-415.

Smith, S.M., Jenkinson, M., Woolrich, M.W., Beckmann, C.F., Behrens, T.E.J., JohansenBerg, H., Bannister, P.R., De Luca, M., Drobnjak, I., Flitney, D.E., Niazy, R.K., Saunders, J., Vickers, J., Zhang, Y., De Stefano, N., Brady, J.M., Matthews, P.M., 2004. Advances in functional and structural MR image analysis and implementation as FSL. Neuroimage 23 (Suppl. 1), S208-S219. 
Sparing, R., Mottaghy, F.M., 2008. Noninvasive brain stimulation with transcranial magnetic or direct current stimulation (TMS/tDCS) - from insights into human memory to therapy of its dysfunction. Methods 44, 329-337.

Thielscher, A., Kammer, T., 2002. Linking physics with physiology in TMS: a sphere field model to determine the cortical stimulation site in TMS. Neuroimage 17, 1117-1130.

Thielscher, A., Kammer, T., 2004. Electric field properties of two commercial figure-8 coils in TMS: calculation of focality and efficiency. Clin. Neurophysiol. 115, 1697-1708.

Thielscher, A., Opitz, A., Windhoff, M., 2011. Impact of the gyral geometry on the electric field induced by transcranial magnetic stimulation. Neuroimage 54, 234-243.

Thomson, A.M., 2003. Presynaptic frequency- and pattern-dependent filtering. J. Comput. Neurosci. 15, 159-202.

Toschi, N., Welt, T., Guerrisi, M., Keck, M.E., 2008. A reconstruction of the conductive phenomena elicited by transcranial magnetic stimulation in heterogeneous brain tissue. Phys. Med. 24, 80-86.

Wagner, T., Fregni, F., Eden, U., Ramos-Estebanez, C., Grodzinsky, A., Zahn, M., PascualLeone, A., 2006. Transcranial magnetic stimulation and stroke: a computer-based human model study. Neuroimage 30, 857-870.
Wagner, T., Valero-Cabre, A., Pascual-Leone, A., 2007. Noninvasive human brain stimulation. Annu. Rev. Biomed. Eng. 9, 527-565.

Wassermann, E.M., Zimmermann, T., 2012. Transcranial magnetic brain stimulation: therapeutic promises and scientific gaps. Pharmacol. Ther. 133, 98-107.

Weiss, C., Nettekoven, C., Rehme, A.K., Neuschmelting, V., Eisenbeis, A., Goldbrunner, R. Grefkes, C., 2012. Mapping the hand, foot and face representations in the primary motor cortex - retest reliability of neuronavigated TMS versus functional MRI Neuroimage 66C, 531-542.

Windhoff, M., Opitz, A., Thielscher, A., 2013. Electric field calculations in brain stimulation based on finite elements: an optimized processing pipeline for the generation and usage of accurate individual head models. Hum. Brain Mapp. 34 (4), 923-935 (April).

Yousry, T.A., Schmid, U.D., Alkadhi, H., Schmidt, D., Peraud, A., Buettner, A., Winkler, P., 1997. Localization of the motor hand area to a knob on the precentral gyrus. A new landmark. Brain 120 (Pt 1), 141-157.

Ziemann, U., Paulus, W., Nitsche, M.A., Pascual-Leone, A., Byblow, W.D., Berardelli, A. Siebner, H.R., Classen, J., Cohen, L.G., Rothwell, J.C., 2008. Consensus: motor cortex plasticity protocols. Brain Stimul. 1, 164-182.

Zucker, R.S., Regehr, W.G., 2002. Short-term synaptic plasticity. Annu. Rev. Physiol. 64, 355-405. 


\title{
Validating computationally predicted TMS stimulation areas using direct electrical stimulation in patients with brain tumors near precentral regions
}

\author{
Alexander Opitz ${ }^{\mathrm{a}, 1,}{ }^{*}$, Noman Zafar $^{\mathrm{b}, 1}$, Volker Bockermann ${ }^{\mathrm{b}}$, Veit Rohde ${ }^{\mathrm{b}}$, Walter Paulus ${ }^{\mathrm{a}}$ \\ a Department of Clinical Neurophysiology, Georg-August-University, Göttingen, Germany \\ ${ }^{\mathrm{b}}$ Department of Neurosurgery, Georg-August-University, Göttingen, Germany
}

\section{A R T I C L E I N F O}

\section{Article history:}

Received 9 January 2014

Received in revised form 12 March 2014

Accepted 13 March 2014

\section{Keywords:}

Transcranial magnetic stimulation

Motor cortex

Direct electrical stimulation

Finite element method

\begin{abstract}
A B S T R A C T
The spatial extent of transcranial magnetic stimulation (TMS) is of paramount interest for all studies employing this method. It is generally assumed that the induced electric field is the crucial parameter to determine which cortical regions are excited. While it is difficult to directly measure the electric field, one usually relies on computational models to estimate the electric field distribution. Direct electrical stimulation (DES) is a local brain stimulation method generally considered the gold standard to map structure-function relationships in the brain. Its application is typically limited to patients undergoing brain surgery. In this study we compare the computationally predicted stimulation area in TMS with the DES area in six patients with tumors near precentral regions. We combine a motor evoked potential (MEP) mapping experiment for both TMS and DES with realistic individual finite element method (FEM) simulations of the electric field distribution during TMS and DES. On average, stimulation areas in TMS and DES show an overlap of up to $80 \%$, thus validating our computational physiology approach to estimate TMS excitation volumes. Our results can help in understanding the spatial spread of TMS effects and in optimizing stimulation protocols to more specifically target certain cortical regions based on computational modeling.
\end{abstract}

(c) 2014 The Authors. Published by Elsevier B.V. on behalf of Federation of European Biochemical Societies. This is an open access article under the CC BY license (http://creativecommons.org/licenses/by/3.0/).

\section{Introduction}

Since its introduction (Barker et al., 1985) transcranial magnetic stimulation (TMS) became a widely used tool in cognitive and clinical neuroscience to interfere with ongoing brain activity. TMS works by applying a temporally changing magnetic field through a magnetic coil placed on the scalp, thus inducing an electric field in the brain (Barker et al., 1985; Opitz et al., 2011). This electric field acts upon neuronal structures in the brain and can lead to the initiation of action potentials that can for instance result in a motor evoked potential (MEP) when stimulating the motor cortex. The application of repetitive TMS protocols has been shown to be able to excite or inhibit a certain brain region over a time period of several minutes up to an hour and can induce long term potentiation (LTP) and long term depression (LTD) like effects (Fitzgerald et al., 2006). In cognitive studies, TMS is used either to interfere with neural circuits in a temporal precise manner which was called a "virtual lesion" (Pascual-Leone et al.,

\footnotetext{
1 These authors contributed equally.

* Corresponding author.

E-mail address: alexander.opitz@med.uni-goettingen.de (A. Opitz).
}

1999) or to induce longer lasting effects on neuronal activity in a specific area to study its effect on a certain behavior. For all these applications, the spatial specificity of TMS is of major importance and great efforts are employed to accurately target the intended brain area, e.g. by using a neuronavigation system. However, it is still unclear how large the stimulated area is. To what extent does the induced electric field spread to other brain regions and how can one determine the brain area that causes the functionally relevant effect? For brain areas other than the motor or visual cortex there is no direct functional output of the strength or efficacy of the stimulation and therefore, the motor cortex often serves as a brain region for testing and validating stimulation protocols. It is assumed that the findings concerning the mechanisms of action of stimulation observed at the motor cortex are valid, at least partially, for other brain areas as well. The site of stimulation of TMS was explored in many different ways by combining TMS motor mapping experiments with PET (Wassermann et al., 1996) or fMRI (Diekhoff et al., 2011; Terao et al., 1998; Weiss et al., 2012). Recently, studies were combining these methods with computational modeling of the electric field distribution using realistic finite element method (FEM) models. These models make specific predictions about the electric field distribution in the brain during TMS and might be useful in determining stimulation areas (Thielscher et al.,

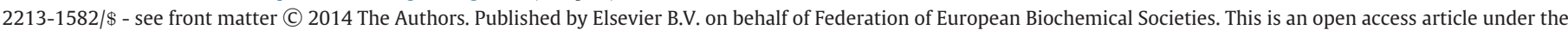
CC BY license (http://creativecommons.org/licenses/by/3.0/).

http://dx.doi.org/10.1016/j.nicl.2014.03.004 
2011). In a recent study by Opitz et al. (2013) it was shown that computationally simulated electric fields were able to predict more than $50 \%$ of the variance of the physiological response in a motor mapping experiment. Furthermore, the combination of physiological MEP data with the modeled electric fields was able to estimate the stimulation area in brain. As the electric field is usually not restricted to a single gyrus but extends to the neighboring gyri as well (Opitz et al., 2011; Thielscher et al., 2011), it would be interesting to know which part of the stimulation area is functionally relevant or what is the threshold necessary to cause a stimulation effect.

One of the most straightforward methods to establish a structurefunction relation in the brain is direct electrical stimulation (DES). Already applied nearly 150 years ago by Fritsch and Hitzig (1870) and later by Ferrier (1876) and Penfield and Boldrey (1937) it was used to establish a detailed somatotopic map of the human cortex. Today, DES is still used to investigate motor behavior, language and cognition (Desmurget et al., 2009; Desmurget et al., 2013). However, its status as a gold standard for mapping brain functions is not unchallenged because of its complex and sometimes even opposite effects at the same stimulation site (Borchers et al., 2012). In neurosurgery DES is widely used to map eloquent motor areas before tumor resection near the precentral gyrus, so that brain tissue crucial for motor control can be preserved during surgery. In many studies, DES has been compared to TMS to test if eloquent motor areas can also be reliably predicted with TMS (Krieg et al., 2012; Picht et al., 2011; Vitikainen et al., 2013) or fMRI (Forster et al., 2011) in a noninvasive manner. Similar approaches are also employed for language mapping (Sollmann et al., 2013; Tarapore et al., 2013). These studies provided valuable insights into the prediction accuracy of TMS for neurosurgical guidance (Picht et al., 2012) and established TMS as a useful tool for presurgical planning. However, the capability to precisely determine stimulated brain areas with TMS in these studies is limited as they are largely relying on either spherical models or projection based approaches to determine the stimulation area of TMS. It has been shown theoretically that these approaches are not able to capture important determining factors of TMS such as brain gyrification or coil orientation and tilts (Opitz et al., 2013; Thielscher et al., 2011).

In this study, we use individualistic high resolution finite element modeling for both TMS and DES to explore the spatial extent of the TMS effect in the brain and show how the combination of TMS with realistic FEM computational modeling can be a powerful tool to noninvasively map structure-function relationships in patients with brain pathologies.

\section{Methods}

\subsection{Subjects}

Six patients ( 3 men, 3 women, ages $44-79$, mean 63.5 years, all right handed) with tumors in the vicinity of the motor cortex were included in the study. Five of the six patients suffered from spreading metastasis from a primary lung tumor. The other patient had an astrocytoma glioblastoma. Written informed consent was obtained before the study. All study procedures were approved by the ethics committee of the University Hospital Göttingen.

\section{Magnetic resonance imaging}

MR images were acquired at $3 \mathrm{~T}$ (Magnetom Trio, Siemens Medical Solutions, Erlangen, Germany) using an eight-channel head coil. Images were acquired mainly for diagnostic purposes including a T1weighted image (MPRAGE, TR $=2000 \mathrm{~ms}, \mathrm{TE}=2.98 \mathrm{~ms}$, flip angle $=9^{\circ}$, $1 \times 1 \times 1.1 \mathrm{~mm}$ resolution) and a T2-weighted image (spin echo, $\mathrm{TR}=3200 \mathrm{~ms}, \mathrm{TE}=458 \mathrm{~ms}, 0.9 \mathrm{~mm}$ isotropic resolution). Furthermore a contrast enhanced MR image $(\mathrm{TR}=4.6 \mathrm{~ms}$, $\mathrm{TE}=1.67 \mathrm{~ms}$, flip angle $=15^{\circ}, 1 \mathrm{~mm}$ isotropic resolution) was acquired to be used for neuronavigation during surgery.

\subsection{TMS motor mapping}

TMS has been conducted using a MagPro X100 stimulator with a C-B60 coil (figure-eight coil, $35 \mathrm{~mm}$ inner diameter, $75 \mathrm{~mm}$ outer diameter, $11 \mathrm{~mm}$ winding height, two layers of 5 windings for each wing of the coil; MagVenture, Inc., Atlanta, Georgia USA). A neuronavigation system (Visor2, ANT, Netherlands) was used to constantly monitor coil position and orientation with respect to the patient's head. Patients were seated comfortably in a reclined chair with head and arm rests. The motor hotspot was determined as the point which consistently resulted in the largest MEPs by moving the coil over the scalp. A $5 \mathrm{~cm} \times 5 \mathrm{~cm}$ rectangular grid (1 cm spacing) centered on the initially determined hotspot was created using custom Matlab scripts (Fig. 1A left panel). Single pulse TMS with 120\% resting motor threshold (RMT) of the first dorsal interosseous (FDI) hand muscle was applied at each grid point. The RMT was defined as the intensity that elicited at least 5 of $10 \mathrm{MEPs}$ of at least $50 \mu \mathrm{V}$ amplitude. In total 10 pulses with an interpulse interval of $4 \mathrm{~s}$ with $400 \mathrm{~ms}$ jitter were applied at each grid point. The coil angle applied during the whole experiment was approximately $45^{\circ}$ to midline and recorded with the neuronavigation system (Fig. 1A right panel).

MEPs were recorded using $\mathrm{Ag} / \mathrm{AgCl}$ bipolar surface electrodes placed over the FDI in a belly-tendon montage. Signals were sampled at $5 \mathrm{kHz}$ and band-pass filtered between $2 \mathrm{~Hz}$ and $2 \mathrm{kHz}$ (Fig. 1B left panel). Analog to Digital conversion was performed with a micro 1401 AD converter (Cambridge Electronic Design, Cambridge, UK). Signals were viewed with Signal 3 (Cambridge Electronic Design, v. 2.13) and stored on a computer for later offline analysis. MEP peak to peak amplitudes were averaged over each grid position and MEP maps were computed (Fig. 1B right panel).

\subsection{Intraoperative direct electrical stimulation}

For each patient a standard neuronavigated craniotomy was performed to get access to tumor regions. Intraoperative neuromonitoring was performed before and during operation using an Endeavor CR neuromonitoring unit (Viasys, Nicolet Biomedical, Dublin/Ohio, USA) with a monopolar brain-stimulation electrode (1.3 $\mathrm{mm}$ diameter, Inomed, Germany). Nine points on the surface of the precentral gyrus (with ca. $5 \mathrm{~mm}$ spacing, see Fig. 2A) spanning a cortical surface area of $2-4 \mathrm{~cm}^{2}$ were stimulated with a single anodal square pulse (pulse duration $0.2 \mathrm{~ms}$ ). In two patients only five points could be stimulated due to constraints during the surgery. However for both patients, enough MEPs were elicited which showed a spatial variation over the stimulation points. For an illustration of the neuronavigated direct electrical stimulation procedure see Fig. 2B. Current intensities of $5 \mathrm{~mA}, 10 \mathrm{~mA}$ and maximally $20 \mathrm{~mA}$ if no response was achieved with lower intensities were applied. In addition, responses from two reference points which were located ca. $2 \mathrm{~cm}$ away from the other points were recorded. MEPs of the FDI hand muscle that were elicited by DES were recorded and stored offline for further analysis.

\subsection{Computational modeling}

\subsubsection{Realistic FEM model}

For each patient an individual FEM model (Fig. 3A left panel) based on the T1- and T2- images was constructed using SimNibs (Windhoff et al., 2013). Failures in the automated FEM creation including missegmentations and failures in the meshing process were corrected manually when necessary. Tumor tissue in regions near the motor cortex was segmented from the MR images by intensity thresholding and corrected manually. Electric field simulations were performed in 

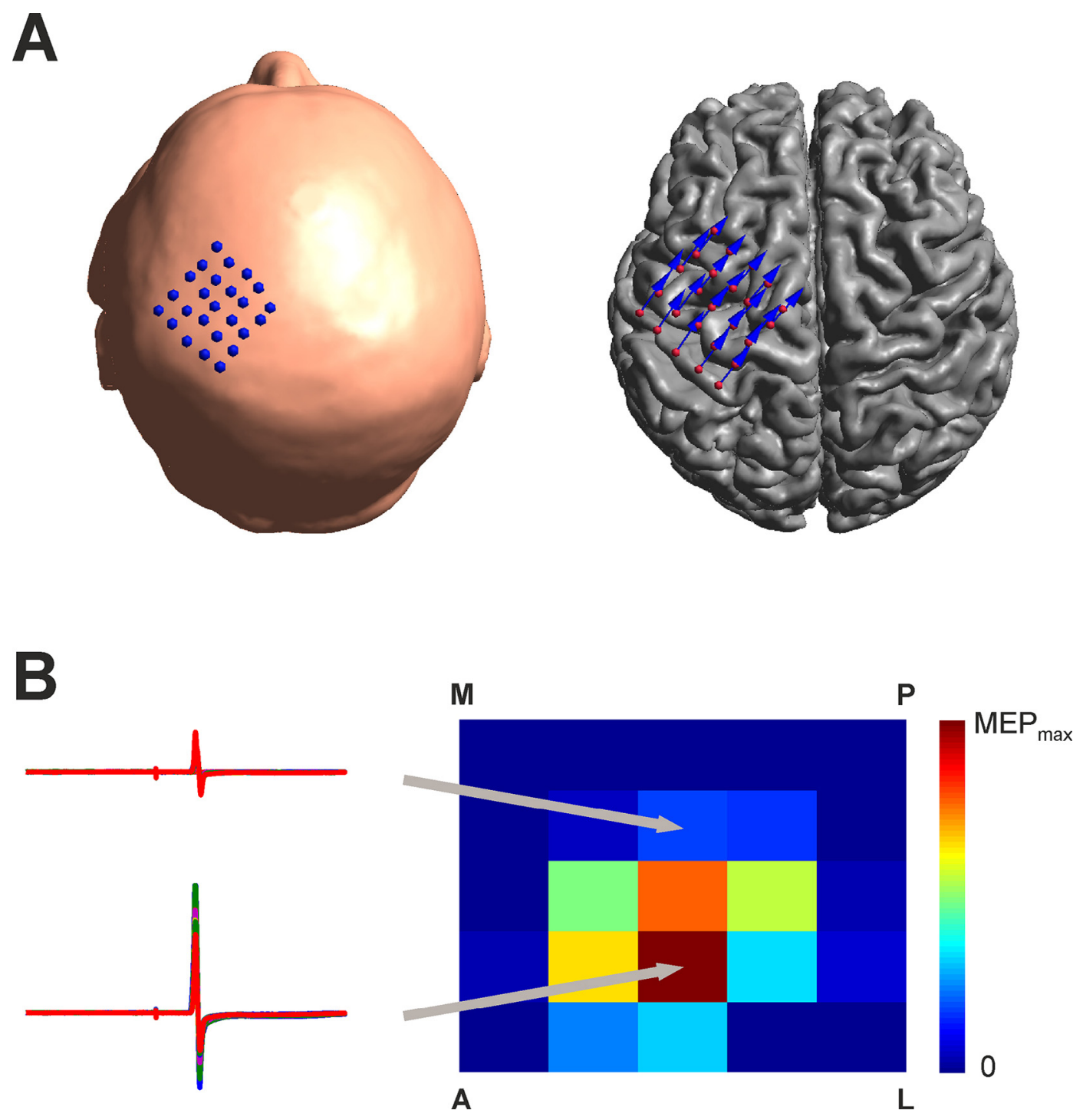

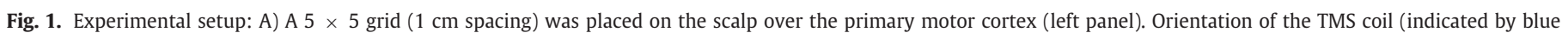

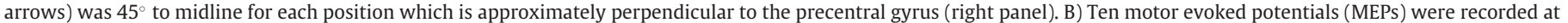
each position (overlaid potentials for two positions shown at the left panel). Based on the average of the MEP amplitudes a MEP map is calculated.

SimNibs for each coil position recorded during the TMS neuronavigated experiment (for an example see Fig. 3B left panel). Isotropic conductivities were used as follows: $\sigma_{\text {skin }}=0.465 \mathrm{~S} / \mathrm{m}, \sigma_{\text {skull }}=0.010 \mathrm{~S} /$ $\mathrm{m}, \sigma_{\mathrm{CSF}}=1.654 \mathrm{~S} / \mathrm{m}, \sigma_{\mathrm{GM}}=0.276 \mathrm{~S} / \mathrm{m}$, and $\sigma_{\mathrm{WM}}=0.126 \mathrm{~S} / \mathrm{m}$. Tumor conductivity was set to the conductivity of the surrounding WM tissue. We chose this value as most tumors consisted of lung tissue which has nearly the same conductivity as WM (Gabriel et al., 1996) and edemas around the tumor which might have higher conductivities were less pronounced among the patients. However, as a precise conductivity value for the tumors is hard to determine, we investigated the effect of varying tumor conductivities in more detail in one subject (see Supplementary material). Based on the simulation results, a MEP weighted mean electric field ( $\left.E_{\text {CoG_realistic }}\right)$ distribution was computed as described in Opitz et al. (2013). The rationale behind this method is that those electric field distributions that resulted in strong MEPs were stimulating functionally important brain areas while those which resulted in weak MEPs were only weakly stimulating functionally relevant brain regions.

\subsubsection{Spherical model}

The prediction accuracy of the realistic FEM model was compared with that of a spherical model (Fig. 3A right panel) for each subject. To that end, a 5 layer spherical model as described in Thielscher et al. (2011) was fitted to the upper half of the skin surface using an ordinary least squares method (Nummenmaa et al., 2013). The upper skin surface was chosen for fitting, as it best resembles a spherical surface compared to the other tissue types. The radii (mean \pm SD in $\mathrm{mm}$ ) of the different tissues were $r_{\text {skin }}=88.8 \pm 3.9, r_{\text {skull }}=84.8 \pm 4.1$, $r_{\mathrm{csf}}=77.8 \pm 4.4, r_{\mathrm{gm}}=74.0 \pm 4.8, \mathrm{r}_{\mathrm{wm}}=69.8 \pm 5.0$. Based on the spherical model, electric field distributions were computed for all coil positions (see Fig. 3B right panel for an example). Also, an MEP weighted mean electric field distribution ( $\left.E_{\text {CoG_sphere }}\right)$ was computed for the spherical model. To compare the results between the spherical and the realistic model the values at the nodes of the spherical model in the GM and WM volume were interpolated to the realistic GM surface using a nearest neighbor approach. 


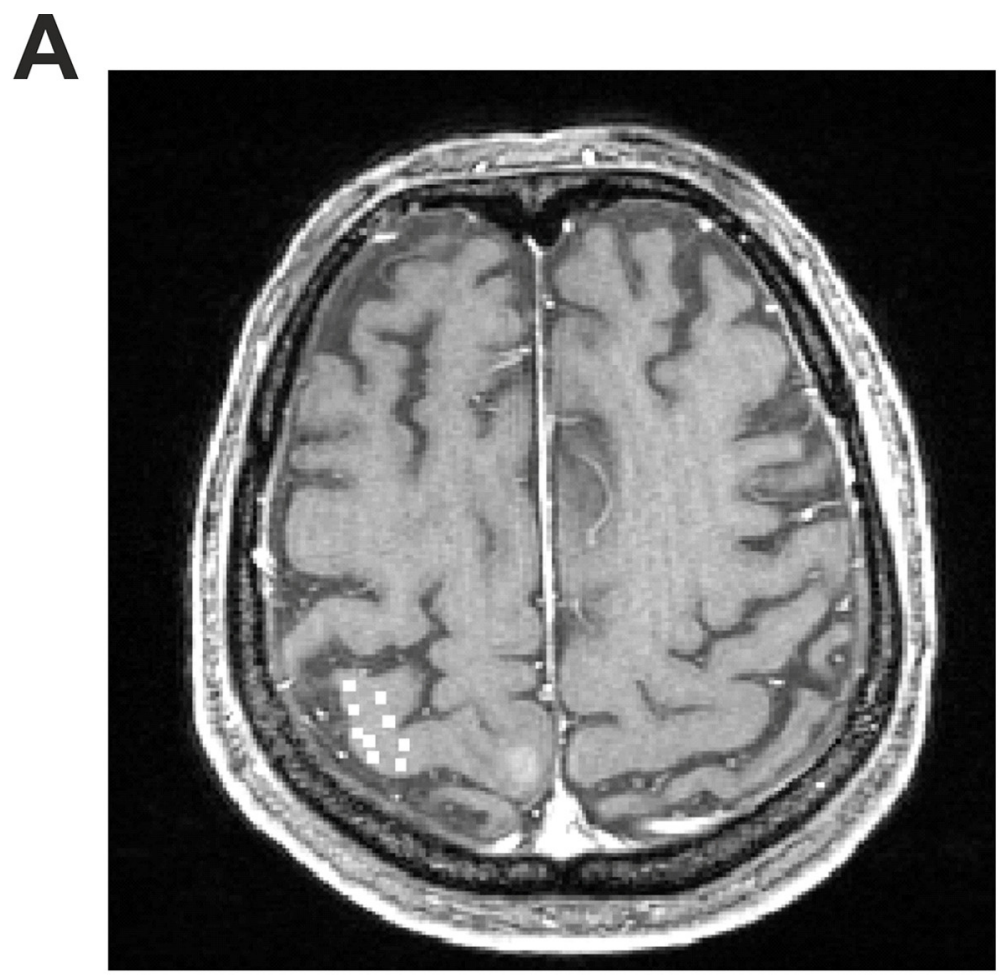

B
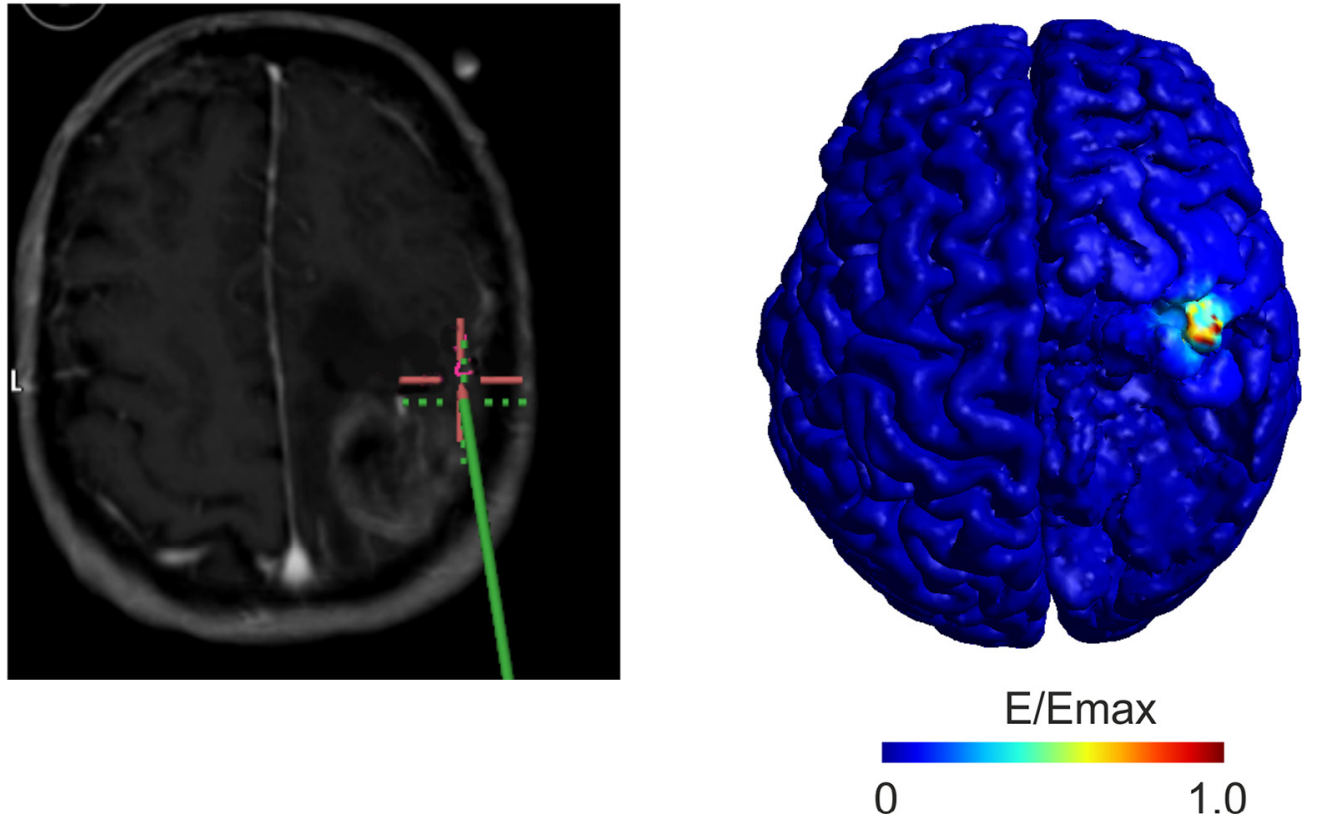

Fig. 2. Direct electrical stimulation: A) Shown are the DES stimulation points (white squares enhanced in size for better visibility) in one example subject. B) Illustration of the intraoperative stimulation procedure. The position of the stimulation electrode is controlled by a neuronavigation software. The red cross indicates the target point at which the stimulation electrode was aimed (green cross). Different points on the motor cortex were stimulated and the elicited MEP recorded. C) Simulated electric field distribution for the DES for one stimulation point. High electric field strengths are restricted to a confined radius around the stimulation electrode. 
A
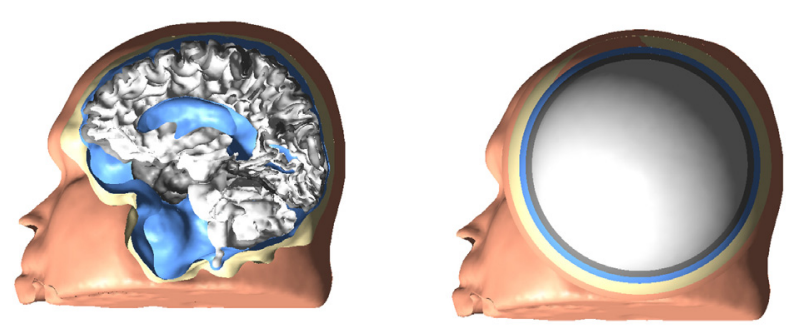

B

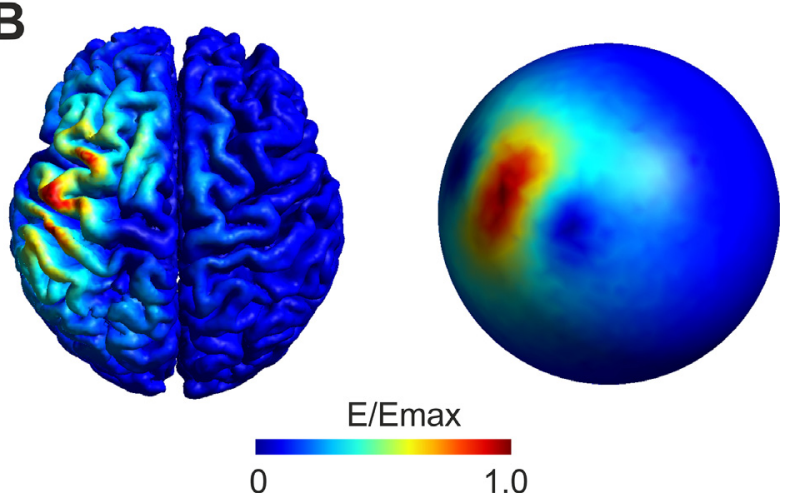

Fig. 3. TMS computational models: A) sagittal cut through the head models for both the realistic (left panel) and the spherical (right panel) case. The surfaces of the five different tissue types are shown. The spherical model was fitted to the upper half of the skin surface of the realistic model. B) Exemplary electric field distribution in one patient for one coil position for both the realistic (left panel) and the spherical (right panel) model. While in the realistic model clear effects of tissue boundaries are visible, the electric field distribution of the spherical model is mainly determined by the primary electric field of the TMS coil.

\subsubsection{DES simulation}

In addition, the electric field during DES was simulated using the realistic head models. The monopolar electrical stimulation was modeled by applying a Dirichlet boundary condition (Joucla and Yvert, 2012) for the electric potential at the stimulation point at the GM surface and a remote large return electrode at the inferior end of the FEM model. An example of the DES electric field is shown in Fig. 2C. The average electric field weighted by the MEPs recorded during surgery ( $\left.E_{\text {COG_DES }}\right)$ was computed the same way as described for the TMS induced electric field.

\subsubsection{TMS-DES comparison}

To compare the extent of the simulated TMS electric field stimulation area that coincides with the DES stimulation area we computed the percentage of the area on the GM surface of the $E_{\text {CoG_realistic }}$ included in the area of the $E_{C O G D E S}$. First, we determined a DES ROI by thresholding the $E_{C O G D D E S}$ at $30 \%$ of its maximum (for an illustration of the DES ROI see Fig. 4D). This threshold was chosen as the $E_{\text {COG_DES }}$ drops off fairly steep and with this chosen threshold a good coverage of the handknob region of the motor cortex was achieved. However, this choice of threshold is arbitrary and therefore we performed the same calculation with different thresholds ranging from $10 \%$ to $90 \%$ (see Supplementary material) to check the robustness of this method. In a second step, we determined the area that was covered by the $E_{\text {CoG_realistic }}$ exceeding a certain threshold of its maximum. We computed this area in $10 \%$ steps from $10 \%$ to $90 \%$ of the electric field maximum and determined the overlap with the DES ROI. Based on the overlap area we determined the percentage of the thresholded $E_{\text {CoG_realistic }}$ included in the DES ROI. The same analysis was performed
A

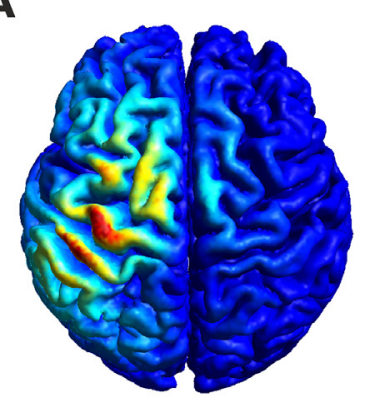

B

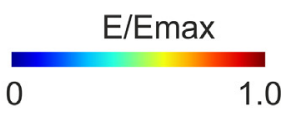

C

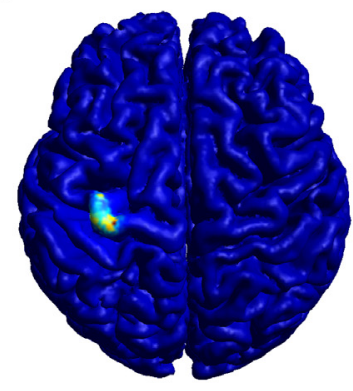

D

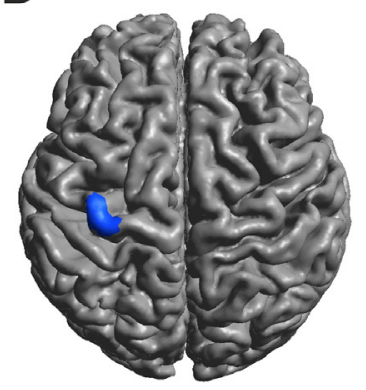

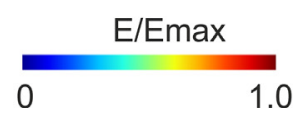

Fig. 4. Computational predicted stimulation areas: Shown is the MEP weighted mean electric field for the A) TMS realistic model, B) TMS spherical model interpolated on the realistic GM surface and C) DES. The stimulation area in the realistic model is restricted to the crowns of the precentral gyrus as well as neighboring gyri. For the spherical model stimulation area is more extended. The stimulation area of the DES is mostly restricted to the primary motor cortex. D) Region of interest (blue area) based on the DES stimulation area (MEP weighted mean electric field $>30 \%$ of its maximum field strength).

with the $E_{\text {CoG_sphere }}$ to compare the prediction accuracy of both models. The rationale for this method is that the higher the $E_{\text {CoG_realistic }}$ is, the more likely it should be functionally effective and stimulate a brain area that has a causal role for generating MEPs. With higher electric field strength threshold, this area should be located in the DES ROI which serves as a gold standard for determining causal relevant areas for generating MEPs.

In a second analysis step we computed the center of gravities of the $E_{\text {CoG_realistic }}$ map, the $E_{\text {CoG_sphere }}$ map and the $E_{\text {COG_DES }}$ map (in its $30 \%$ threshold ROI). This method reduces the electric field maps to one single point. In the following the Euclidian distance between these CoG points was computed. As the determination of these points also depends on the chosen electric field threshold, we again computed the distances in $10 \%$ steps from $10 \%$ to $90 \%$ of the TMS electric field maximum.

Finally, in order to acquire a simple model free estimate of the DES CoG, we made the zero order approximation that DES causes a point like excitation at the tip of the stimulation electrode. Based on this method we recomputed the DES center of gravity and the Euclidian distances to the TMS center of gravities for the realistic and spherical model.

All the described computations were performed for each subject individually and mean and standard error of mean were calculated over all subjects subsequently. 
Table 1

Covered cortical surface area (mean \pm standard error of mean over six patients) in $\mathrm{cm}^{2}$ depending on the threshold of the $E_{C o G}$ measured on the realistic model.

\begin{tabular}{lll}
\hline $\begin{array}{l}\text { Electric field threshold } \\
\text { in percent of the } \\
\text { maximum }\end{array}$ & Spherical model & Realistic model \\
\hline $10 \%$ & $(9.68 \pm 0.29) \times 10^{2}$ & $(4.67 \pm 0.41) \times 10^{2}$ \\
$20 \%$ & $(3.74 \pm 0.16) \times 10^{2}$ & $(1.57 \pm 0.18) \times 10^{2}$ \\
$30 \%$ & $(1.69 \pm 0.11) \times 10^{2}$ & $(7.15 \pm 0.85) \times 10^{1}$ \\
$40 \%$ & $(9.35 \pm 0.52) \times 10^{1}$ & $(3.74 \pm 0.49) \times 10^{1}$ \\
$50 \%$ & $(5.38 \pm 0.30) \times 10^{1}$ & $(1.94 \pm 0.27) \times 10^{1}$ \\
$60 \%$ & $(3.22 \pm 0.19) \times 10^{1}$ & $(1.01 \pm 0.13) \times 10^{1}$ \\
$70 \%$ & $(1.77 \pm 0.10) \times 10^{1}$ & $(4.85 \pm 0.64) \times 10^{0}$ \\
$80 \%$ & $(7.07 \pm 0.89) \times 10^{0}$ & $(1.81 \pm 0.33) \times 10^{0}$ \\
$90 \%$ & $(1.98 \pm 0.38) \times 10^{0}$ & $(0.59 \pm 0.15) \times 10^{0}$ \\
\hline
\end{tabular}

\section{Results}

Both the $E_{\text {CoG_realistic }}$ and the interpolated $E_{\text {CoG_sphere }}$ show high electric field strength in M1 (Fig. 4A and B for one example patient). For the realistic model, high electric field strengths were restricted to gyral crowns and were rapidly decreasing with increasing sulcal depth. The stimulation area was restricted to a confined region for the realistic model while for the spherical model a larger area exhibited high electric field strength. The larger stimulation area for the interpolated spherical model was independent from the applied threshold (see Table1). The $E_{\text {COGDES }}$ was considerably more spatially restricted (Fig. 4C for one example patient) towards the gyral crown of M1.

To analyze the mapping accuracy of the TMS computational model we computed the percentage of the area of the $E_{C o G \text { realistic in a DES }}$ determined region of interest (Fig. 4D for an example) for different electric field thresholds. By increasing the threshold, a higher percentage of the electric field was included in the DES ROI (Fig. 5A). For the realistic model about $80 \%$ of its highest $E_{\text {CoG_realistic }}(>90 \%$ of the maximum) area fell into the DES ROI. In comparison, for the spherical model a smaller percentage of its highest $E_{C O G}$ sphere was included in the DES ROI. The differences between the realistic and the spherical model were mainly due to the larger stimulation area determined by the spherical model (see Table 1 for the covered area on the brain for both the interpolated spherical and the realistic model). The better overlap of the realistic compared to the spherical model was independent of the threshold chosen to determine the DES ROI (Supplementary Fig. 1). A similar trend as for the stimulation area overlap between DES and TMS was found for the Euclidian distances. With increasing electric field threshold, the Euclidian distance for the TMS map CoG to the DES map CoG decreased (Fig. 5B). For the $90 \%$ threshold the distance was $6.3 \pm 0.7 \mathrm{~mm}$ for the realistic and $8.9 \pm 1.7 \mathrm{~mm}$ for the spherical model. For the model free simple estimate of the DES CoG the Euclidian distances to the TMS map CoGs were $9.4 \pm 1.5 \mathrm{~mm}$ for the realistic and $11.0 \pm 1.5 \mathrm{~mm}$ for the spherical model for the $90 \%$ threshold, respectively.

\section{Discussion}

In this study we investigated the mapping accuracy of transcranial magnetic stimulation for determining motor areas by comparing it to the gold standard of direct electrical stimulation. We found that the TMS stimulation area determined by a computational approach significantly overlaps with the computed DES stimulation area (see Figs. 4 and 5). Especially in the area of highest electrical field strength, the overlap was strongest. Also the distance between the TMS map CoG and the DES map CoG was shortest for the highest electric field strengths. Using a simplified approach which does not depend on a computational model to estimate the DES CoG results in slightly larger distances, which indicates that a merely point like stimulation approximation in DES might not be appropriate but nevertheless
A

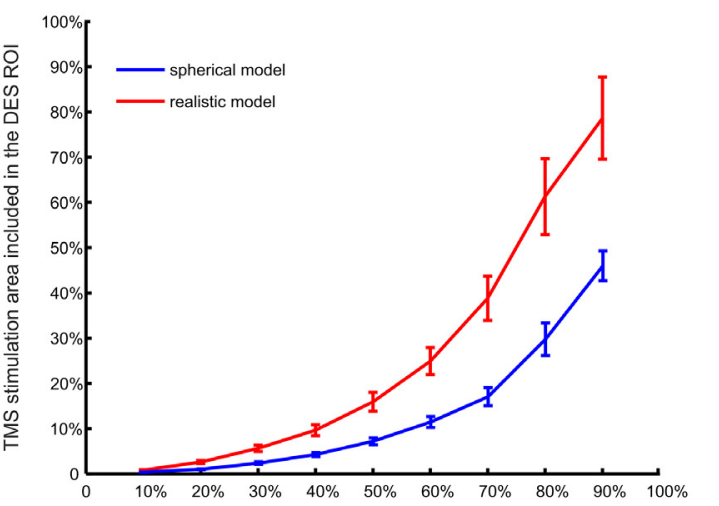

Threshold of the TMS stimulation area in percent of the maximum electric field

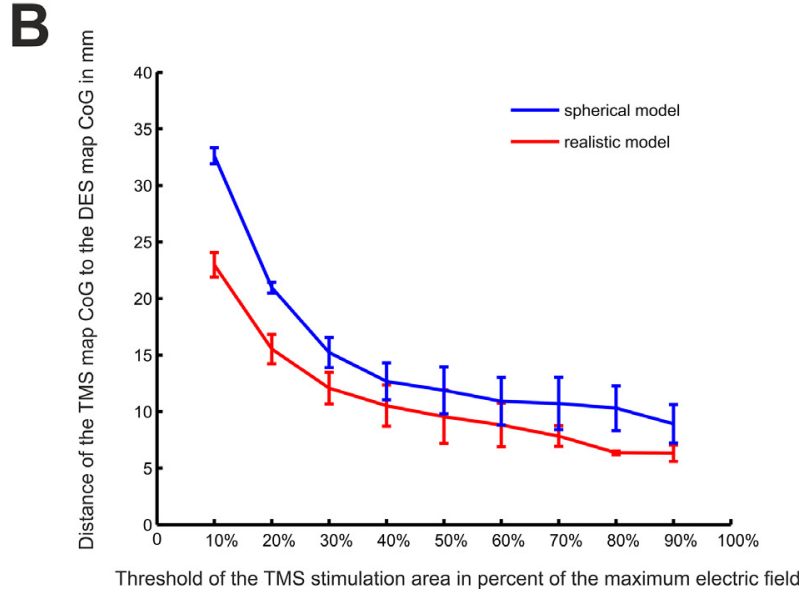

Fig. 5. DES and TMS comparison: A) percentage of the overlap between the DES stimulation area $\left(3.99 \pm 0.46 \mathrm{~cm}^{2}\right)$ and the stimulation area of the TMS for both the realistic model (red line) and the spherical model (blue line). Shown are mean \pm standard error of mean over the six patients for the overlap between the DES and TMS stimulation areas for different thresholds of the TMS electric field. For increasing TMS electric field strengths an increasing percentage overlaps with the DES ROI. This effect is more pronounced for the realistic model than for the spherical model. B) Distance between the CoG of the TMS map and the CoG of the DES map for both the realistic and the spherical model. Shown are mean \pm standard error of mean over the six patients for the distance between the DES and TMS CoGs for different thresholds of the TMS electric field. With increasing TMS electric field threshold, the distance between the TMS and DES CoGs decreases.

can give another validation independent of the TMS computational model.

Compared to spherical models, realistic models make a more specific prediction of TMS target areas which are in better accordance with the DES results. Spherical models are not able to account for effects caused by the gyrification of the brain. The secondary electric field caused by charge accumulation at the tissue interface between CSF and GM can have a profound influence on the electric field distribution mainly leading to enhanced electric fields at the gyral crowns perpendicular to the coil orientation (Thielscher et al., 2011). Thus, taking into account the gyrification of the brain surface causes a spatially more specific prediction than that of a spherical model. The superior overlap of the realistic model compared to the spherical model with the DES stimulation area indicates that for a precise estimation of TMS target areas an accurate representation of the cortical foldings seems necessary. The good overlap of the TMS stimulation area of the realistic model with the DES gold standard also validates the use of individual FEM models as being able to reliably predict brain regions excited by TMS. While in this study we focused on the motor cortex, potentially TMS FEM simulations might also help in predicting stimulation sites that are non-motor related, for example brain regions 
that are related to speech.

While it is difficult to put an exact number to the spatial extent of the excitation area of DES and TMS, a few estimates can be made. The radius of the current spread of direct electrical stimulation can be estimated by the formula: $I=K r^{2}$ (Ranck, 1975; Stoney et al., 1968), with $I$ the current strength in $\mu \mathrm{A}, K$ the current-distance constant which was estimated to be $K=1292 \mu \mathrm{A} / \mathrm{mm}^{2}$ by Stoney et al. (1968) and $r$ the radius of the stimulation area in $\mathrm{mm}$. Based on this formula, the stimulation radius can be estimated to lie between 2 and $4 \mathrm{~mm}$ for current strengths between 5 and $20 \mathrm{~mA}$ as applied in this study. This might lie in a similar range as has been estimated for deep brain stimulation (DBS) (McIntyre et al., 2004). However, the effective stimulation area of DES might be a factor of two or three larger than estimated as was shown by combining microstimulation with fMRI (Tolias et al., 2005). Despite this, the stimulation area of DES seems to be in the range of a few $\mathrm{mm}^{2}$ while the predicted stimulation area of TMS spans several $\mathrm{cm}^{2}$ which can extend over one or two neighboring gyri. Thus, a millimeter precise stimulation of cortical tissue in a noninvasive manner does not seem possible with currently employed TMS coils. Other noninvasive brain stimulation methods like transcranial focused ultrasound (Legon et al., 2014; Tufail et al., 2010) stimulation might be able to overcome the physical constraints of transcranial magnetic stimulation.

One possible limitation of this study is that the area that was directly stimulated during surgery was limited in its spatial extent. As only as much skull and dura was removed as was necessary to have access to the tumor, the brain area that was accessible with DES was limited. Thus, the estimated DES stimulation area might be larger than that estimated by the applied procedure. However, reference points that were recorded further apart from the motor areas did not elicit any MEPs even with highest stimulation amplitudes. Also, in every patient there were DES stimulation points that did not result in MEPs at all, thus demonstrating that there was a spatial confinement in the measurement data. Another point we cannot address in this study is the possible influence of neuronal elements deeper in the sulcal wall as they were not accessible with the applied preparation during surgery. Also we did not take conductivity anisotropy into account which exerts stronger effects in deeper WM regions but is likely to be negligible in superficial GM (Opitz et al., 2011). Thus, we deliberately focused on comparing the surface effects of TMS and DES. The influence of the tumor on the electric field distribution resulted in nonnegligible changes only in the case of very high tumor conductivities, which likely did not occur in our study. Although it seems unlikely that our results are significantly dependent on tumor conductivity, future studies might profit from a direct conductivity measure of the tumor using Magnetic Resonance Impedance Tomography (Minhas et al., 2011). The mapping accuracy of TMS can be possibly improved by employing more than one TMS coil orientation which was not performed in this study to keep the experimental time for the patients as short as possible. Locally enhanced mesh resolution around the stimulation electrode can improve the numerical precision of the DES results in future studies. Finally, mapping accuracy depends on the precision of the neuronavigation procedure which is estimated to have an uncertainty of ca. $5 \mathrm{~mm}$ (Ruohonen and Karhu, 2010).

Future studies can possibly improve on predicting the stimulation area by taking into account other factors like the orientation and morphology of the targeted neuronal elements (Radman et al., 2009) which determine their degree of excitability by external electric fields as was already addressed in Salvador et al. (2011) or Pashut et al. (2011). However, in the absence of precise knowledge of these factors the absolute electric field strength seems to be a robust measure to estimate stimulation areas. In this study we focused on the direct stimulation effects of the electric field, however it is generally assumed that TMS acts on brain circuits (Dayan et al., 2013; Fox et al., 2012) and exerts its effect on remote brain areas as well. In conclusion, our results suggest that TMS combined with computational electric field modeling can reliably predict stimulation areas that show large overlap with computationally predicted DES areas. Even though the more commonly employed spherical models do not miss the stimulation area, their spatial specificity is limited compared to more realistic models. This computational approach can possibly be used to more precisely determine eloquent motor areas in a noninvasive manner before brain surgery. It has to be tested in future studies if this method can help to improve clinical outcomes for patients undergoing brain tumor resection.

\section{Supplementary material}

Supplementary material associated with this article can be found, in the online version, at doi:10.1016/j.nicl.2014.03.004.

\section{References}

Barker, A.T., Jalinous, R., Freeston, I.L., 1985. Non-invasive magnetic stimulation of human motor cortex. Lancet 1, 1106-7, 2860322.

Borchers, S., Himmelbach, M., Logothetis, N., Karnath, H.O., 2012. Direct electrical stimulation of human cortex - the gold standard for mapping brain functions? Nature Reviews. Neuroscience 13, 63-70, 22127300.

Dayan, E., Censor, N., Buch, E.R., Sandrini, M., Cohen, L.G., 2013. Noninvasive brain stimulation: from physiology to network dynamics and back. Nature Neuroscience 16, 838-44. http://dx.doi.org/10.1038/nn.3422, 23799477.

Desmurget, M., Reilly, K.T., Richard, N., Szathmari, A., Mottolese, C., Sirigu, A. 2009. Movement intention after parietal cortex stimulation in humans. Science (New York, N.Y.) 324, 811-13. http://dx.doi.org/10.1126/science.1169896, 19423830.

Desmurget, M., Song, Z., Mottolese, C., Sirigu, A., 2013. Re-establishing the merits of electrical brain stimulation. Trends in Cognitive Sciences 17, 442-9. http://dx.doi. org/10.1016/j.tics.2013.07.002, 23932195.

Diekhoff, S., Uludağ, K., Sparing, R., Tittgemeyer, M., Cavuşoğlu, M., von Cramon, D.Y. et al, 2011. Functional localization in the human brain: gradient-echo, spin-echo, and arterial spin-labeling fMRI compared with neuronavigated TMS. Human Brain Mapping 32, 341-57. http://dx.doi.org/10.1002/hbm.21024, 20533563.

Ferrier, D., 1876. Functions of the Brain. Putnam.

Fitzgerald, P.B., Fountain, S., Daskalakis, Z.J., 2006. A comprehensive review of the effects of rTMS on motor cortical excitability and inhibition. Clinical Neurophysiology: Official Journal of the International Federation of Clinical Neurophysiology 117, 2584-96. http://dx.doi.org/10.1016/j.clinph.2006.06.712, 16890483.

Forster, M.T., Hattingen, E., Senft, C., Gasser, T., Seifert, V., Szelényi, A., 2011. Navigated transcranial magnetic stimulation and functional magnetic resonance imaging: advanced adjuncts in preoperative planning for central region tumors. Neurosurgery 68, 1317-24, discussion: 1324-1315 21273929.

Fox, M.D., Halko, M.A., Eldaief, M.C., Pascual-Leone, A., 2012. Measuring and manipulating brain connectivity with resting state functional connectivity magnetic resonance imaging (fcMRI) and transcranial magnetic stimulation (TMS). Neuroimage 62, 2232-43. http://dx.doi.org/10.1016/j.neuroimage.2012.03.035, 22465297.

Fritsch, G., Hitzig, E., 1870. Über die elektrische Erregbarkeit des Grosshirns. Archiv für Anatomie, Physiologie und Wissenschaftliche Medicin 37, 300-32.

Gabriel, S., Lau, R.W., Gabriel, C., 1996. The dielectric properties of biological tissues: III. Parametric models for the dielectric spectrum of tissues. Physics in Medicine and Biology 41, 2271-93. http://dx.doi.org/10.1088/0031-9155/41/11/ 003, 8938026 .

Joucla, S., Yvert, B., 2012. Modeling extracellular electrical neural stimulation: from basic understanding to MEA-based applications. Journal of Physiology, Paris 106, 146-58. http://dx.doi.org/10.1016/j.jphysparis.2011.10.003, 22036892.

Krieg, S.M., Shiban, E., Buchmann, N., Gempt, J., Foerschler, A., Meyer, B. et al, 2012. Utility of presurgical navigated transcranial magnetic brain stimulation for the resection of tumors in eloquent motor areas. Journal of Neurosurgery 116, 9941001. http://dx.doi.org/10.3171/2011.12.JNS111524, 22304452.

Legon, W., Sato, T.F., Opitz, A., Mueller, J., Barbour, A., Williams, A. et al, 2014. Transcranial focused ultrasound modulates the activity of primary somatosensory cortex in humans. Nature Neuroscience 17, 322-9. http://dx.doi.org/10.1038/nn.3620, 24413698.

McIntyre, C.C., Mori, S., Sherman, D.L., Thakor, N.V., Vitek, J.L., 2004. Electric field and stimulating influence generated by deep brain stimulation of the subthalamic nucleus. Clinical Neurophysiology: Official Journal of the International Federation of Clinical Neurophysiology 115, 589-95. http://dx.doi.org/10.1016/j.clinph.2003.10. 033, 15036055.

Minhas, A.S., Jeong, W.C., Kim, Y.T., Han, Y., Kim, H.J., Woo, E.J., 2011. Experimental performance evaluation of multi-echo ICNE pulse sequence in magnetic resonance electrical impedance tomography. Magnetic Resonance in Medicine: Official Journal of the Society of Magnetic Resonance in Medicine / Society of Magnetic Resonance in Medicine 66, 957-65. http://dx.doi.org/10.1002/mrm.22872, 21442654. 
Nummenmaa, A., Stenroos, M., Ilmoniemi, R.J., Okada, Y.C., Hämäläinen, M.S., Raij, T. 2013. Comparison of spherical and realistically shaped boundary element head models for transcranial magnetic stimulation navigation. Clinical Neurophysiology: Official Journal of the International Federation of Clinical Neurophysiology 124, 1995-2007. http://dx.doi.org/10.1016/j.clinph.2013.04.019, 23890512.

Opitz, A., Legon, W., Rowlands, A., Bickel, W.K., Paulus, W., Tyler, W.J., 2013. Physiological observations validate finite element models for estimating subject-specific electric field distributions induced by transcranial magnetic stimulation of the human motor cortex. NeuroImage 81, 253-64. http://dx.doi.org/10.1016/j.neuroimage. 2013.04.067, 23644000.

Opitz, A., Windhoff, M., Heidemann, R.M., Turner, R., Thielscher, A., 2011. How the brain tissue shapes the electric field induced by transcranial magnetic stimulation. Neuroimage 58, 849-59. http://dx.doi.org/10.1016/j.neuroimage.2011.06. 069, 21749927.

Pascual-Leone A. Bartres-Faz D. Keenan, J. . 1999. Transcranial magnetic stimulation: studying the brain-behaviour relationship by induction of 'virtual lesions'. Philosophical Transactions of the Royal Society of London. Series B, Biological Sciences 354, 1229-38. http://dx.doi.org/10.1098/rstb.1999.0476, 10466148.

Pashut, T., Wolfus, S., Friedman, A., Lavidor, M., Bar-Gad, I., Yeshurun, Y. et al, 2011. Mechanisms of magnetic stimulation of central nervous system neurons. PLoS Computational Biology 7. http://dx.doi.org/10.1371/journal.pcbi.1002022, 21455288.

Penfield, W., Boldrey, E., 1937. Somatic motor and sensory representation in the cerebral cortex of man as studied by electrical stimulation. Brain 60, 389-443. http://dx.doi.org/10.1093/brain/60.4.389.

Picht, T., Schmidt, S., Brandt, S., Frey, D., Hannula, H., Neuvonen, T. et al, 2011. Preoperative functional mapping for rolandic brain tumor surgery: comparison of navigated transcranial magnetic stimulation to direct cortical stimulation. Neurosurgery 69, 581-8. http://dx.doi.org/10.1227/NEU.0b013e3182181b89, discussion: 58821430587.

Picht, T., Schulz, J., Hanna, M., Schmidt, S., Suess, O., Vajkoczy, P., 2012. Assessment of the influence of navigated transcranial magnetic stimulation on surgical planning for tumors in or near the motor cortex. Neurosurgery 70, 1248-56. http://dx.doi. org/10.1227/NEU.0b013e318243881e, discussion: 1256-1247 22127045.

Radman, T., Ramos, R.L., Brumberg, J.C., Bikson, M., 2009Brain Stimulation 2, 215-28. http://dx.doi.org/10.1016/j.brs.2009.03.007, 20161507.

Ranck, J.B. Jr., 1975. Which elements are excited in electrical stimulation of mammalian central nervous system: a review. Brain Research 98, 417-40. http://dx.doi.org/10. 1016/0006-8993(75)90364-9, 1102064.

Ruohonen, J., Karhu, J., 2010. Navigated transcranial magnetic stimulation. Neurophysiologie Clinique $=$ Clinical Neurophysiology 40, 7-17. http://dx.doi.org/10.1016/j. neucli.2010.01.006, 20230931.

Salvador, R. Silva, S. Basser, P., Miranda, P.C, 2011. Determining which mechanisms lead to activation in the motor cortex: a modeling study of transcranial magnetic stimulation using realistic stimulus waveforms and sulcal geometry.
Clinical Neurophysiology: Official Journal of the International Federation of Clinical Neurophysiology 122, 748-58. http://dx.doi.org/10.1016/j.clinph.2010.09.022, 21035390.

Sollmann, N., Picht, T., Mäkelä, J.P., Meyer, B., Ringel, F., Krieg, S.M., 2013. Navigated transcranial magnetic stimulation for preoperative language mapping in a patient with a left frontoopercular glioblastoma. Journal of Neurosurgery 118, 175-9. http: //dx.doi.org/10.3171/2012.9.JNS121053, 23101450.

Stoney, S.D. Jr., Thompson, W.D., Asanuma, H., 1968. Excitation of pyramidal tract cells by intracortical microstimulation: effective extent of stimulating current. Journal of Neurophysiology 31, 659-69, 5711137.

Tarapore, P.E., Findlay, A.M., Honma, S.M., Mizuiri, D., Houde, J.F., Berger, M.S. et al, 2013. Language mapping with navigated repetitive TMS: proof of technique and validation. Neuroimage 82, 260-72. http://dx.doi.org/10.1016/j.neuroimage.2013. 05.018, 23702420.

Terao, Y., Ugawa, Y., Sakai, K., Miyauchi, S., Fukuda, H., Sasaki, Y. et al, 1998. Localizing the site of magnetic brain stimulation by functional MRI. Experimental Brain Research 121, 145-52. http://dx.doi.org/10.1007/s002210050446, 9696383.

Thielscher, A., Opitz, A., Windhoff, M., 2011. Impact of the gyral geometry on the electric field induced by transcranial magnetic stimulation. Neuroimage 54, 23443. http://dx.doi.org/10.1016/j.neuroimage.2010.07.061, 20682353.

Tolias, A.S., Sultan, F., Augath, M., Oeltermann, A., Tehovnik, E.J., Schiller, P.H. et al, 2005. Mapping cortical activity elicited with electrical microstimulation using FMRI in the macaque. Neuron 48, 901-11. http://dx.doi.org/10.1016/j.neuron.2005.11.034, 16364895.

Tufail, Y., Matyushov, A., Baldwin, N., Tauchmann, M.L., Georges, J., Yoshihiro, A. et al, 2010. Transcranial pulsed ultrasound stimulates intact brain circuits. Neuron 66, 681-94. http://dx.doi.org/10.1016/j.neuron.2010.05.008, 20547127.

Vitikainen, A.M., Salli, E., Lioumis, P., Mäkelä, J.P., Metsähonkala, L., 2013. Applicability of nTMS in locating the motor cortical representation areas in patients with epilepsy. Acta Neurochirurgica 155,507-18. http://dx.doi.org/10.1007/s00701-012-1609-5, 23328919.

Wassermann, E.M., Wang, B., Zeffiro, T.A., Sadato, N., Pascual-Leone, A., Toro, C. et al, 1996. Locating the motor cortex on the MRI with transcranial magnetic stimulation and PET. NeuroImage 3, 1-9. http://dx.doi.org/10.1006/nimg.1996.0001, 9345470.

Weiss, C., Nettekoven, C., Rehme, A.K., Neuschmelting, V., Eisenbeis, A., Goldbrunner R. et al, 2012. Mapping the hand, foot and face representations in the primary motor cortex - retest reliability of neuronavigated TMS versus functional MRI. Neuroimage 66C, 531-42, 23116812.

Windhoff, M., Opitz, A., Thielscher, A., 2013. Electric field calculations in brain stimulation based on finite elements: an optimized processing pipeline for the generation and usage of accurate individual head models. Human Brain Mapping 34, 923-35. http://dx.doi.org/10.1002/hbm.21479, 22109746. 


\title{
Anatomical determinants of the electric field during transcranial direct current stimulation: Anatomy may overrun electrode placement
}

\author{
Alexander Opitz ${ }^{1}$, Walter Paulus ${ }^{1}$, Susanne Will ${ }^{2}$, and Axel Thielscher ${ }^{3-5}$ \\ ${ }^{1}$ Department of Clinical Neurophysiology, Georg-August-University, Göttingen, \\ Germany
}

${ }^{2}$ Department of Diagnostic and Interventional Radiology, University Clinics of Tübingen, Germany

${ }^{3}$ Danish Research Centre for Magnetic Resonance, Copenhagen University Hospital Hvidovre, Denmark

${ }^{4}$ Biomedical Engineering Section, Technical University of Denmark, Kgs. Lyngby, Denmark

${ }^{5}$ Max-Planck Institute for Biological Cybernetics, Tübingen, Germany

Correspondence by: Alexander Opitz

Email: alexander.opitz@med.uni-goettingen.de

Ph: +49 551 390-8457 


\begin{abstract}
Transcranial direct current stimulation (tDCS) causes a complex electric current flow in the head which hampers the accurate localization of the stimulated brain areas. In this study we show how various anatomical features shape the electric field distribution in the brain during tDCS. We constructed anatomically realistic finite element (FEM) models of two individual heads including conductivity anisotropy and different skull layers. We simulated a widely employed electrode montage to induce motor cortex plasticity and moved the stimulating electrode over the motor cortex in small steps to examine the resulting changes of the electric field distribution in the underlying cortex. Using a multiple regression model we could identify four key factors that determine the field distribution to a significant extent, namely the thicknesses of the skull and cerebrospinal fluid, the gyral depth and the distance to the electrode edge. These four factors alone account for $\sim 50 \%$ of the spatial variation of the electric field. Further, we demonstrate that individual anatomical factors can lead to stimulation "hotspots" which are partly resistant to electrode positioning. Our results give valuable novel insights in the biophysical foundation of tDCS and highlight the importance to account for individual anatomical factors when choosing an electrode montage design.
\end{abstract}




\section{Introduction}

Transcranial direct current stimulation (tDCS) is a widely used brain stimulation technique with various applications in different areas of neuroscience and clinical research. It is typically employed by attaching two large pad electrodes (with a few centimeters edge length) to the head and passing a small electric current in the range of a few mA through them. It has been shown that tDCS can induce changes on motor cortex excitability (Nitsche and Paulus, 2000) that are dependent on stimulation strength and duration (Nitsche and Paulus, 2001). In slice preparations it has been demonstrated that weak DC fields can shift thresholds for action potential generation and exert an influence on spike timing (Bikson et al., 2004, Radman et al., 2007). While the exact mechanisms of action are still under discussion it is generally agreed that the local electric field and its orientation with respect to neuronal structures is a main determinant of the stimulation effects that differ between various stimulation protocols (Radman et al., 2009, Miranda et al., 2013). As direct measurements of these electric fields are difficult to implement various efforts have been made to estimate the electric field distribution by means of computational modeling. Simulation approaches range from spherical models (Miranda et al., 2006, Datta et al., 2008) to more realistic MRI derived head models (Datta et al., 2009, Sadleir et al., 2010) in order to demonstrate the effects of electrode shape, conductivity anisotropy (Suh et al., 2012), different skull layers (Neuling et al., 2012) or artificial skull openings (Datta et al., 2010) on the injected electric field.

However, few studies have tried to quantify how much the spatial distribution of the electric field on the cortical surface is pre-determined by individual anatomical features. That is, to which extent does individual anatomy in addition (or opposing) to the electrode placement dictate the stimulated brain areas?

In the current study we explore the impact of several anatomical factors such as skull thickness and sulcal depth on the electric field pattern in the brain. We employ anatomically realistic FEM models that are based on MR images and accurately capture the gyrification of gray matter (GM), the conductivity anisotropy of white matter (WM), the thickness of the cerebrospinal fluid (CSF) layer, the different skull layers, the eye 
regions and the nasal cavities. We focus on simulating the electric field distribution that might occur during a standard tDCS experiment aimed at inducing motor cortex plasticity. By investigating the effects of systematic displacements of the electrode position on the electric field distribution, we characterize how anatomical constraints interact with the electrode placement. In particular, we assess how stable the stimulated areas in the brain are when varying electrode positions. We demonstrate that our results are consistent across FEM models of two individual heads and are robust across a wide range of simulated electrode thicknesses and conductivities.

\section{Methods}

MR Data acquisition

Structural (sMRI) and diffusion (dMRI) magnetic resonance images were acquired for two healthy participants (one male, one female, $27 \& 26$ years) without history of neurological or psychiatric diseases. The data was collected using a 3T TIM Trio scanner (Siemens Heathcare, Erlangen, Germany) equipped with a 12-channel head coil at the MPI for Biological Cybernetics (Tübingen, Germany). The study was approved by the local ethics committee of the Medical Faculty of the University of Tübingen and data collection was performed after receiving written informed consent.

T1- and T2-weighted MR images were obtained, both with and without fat suppression. 3D MP-RAGE was used for the T1-weighted images (192 sagittal slices, matrix size = $256 \times 256$, voxel size $=1 \mathrm{~mm}^{3}$, flip angle $9^{\circ}$, TR/TE $/ \mathrm{TI}=2300 / 2.98 / 1100 \mathrm{~ms}$ without fat suppression, TR/TE/TI $=2300 / 4.21 / 1100 \mathrm{~ms}$ with selective water excitation). The T2weighted images consisted of 2D turbo spin echo acquisitions (96 sagittal slices, matrix size $=256 \times 256$, voxel size $=1 \times 1 \times 2 \mathrm{~mm}^{3}$, flip angle $110^{\circ}, \mathrm{TR} / \mathrm{TE}=11990 / 102 \mathrm{~ms}$, turbo factor 11, with and without fat suppression). The signal from subdural fat and the spongy bone of the skull was reduced in the fat suppressed images. This simplified the accurate reconstruction of the border between CSF and skull and helped the accurate segmentation of the eye regions. The dMRI images used a twice refocused SE-EPI sequence $(72$ axial slices, matrix size $=128 \times 128$, voxel size $1.9 \times 1.9 \times 2.1 \mathrm{~mm} 3, \mathrm{TR} / \mathrm{TE}=$ 
10500/105 ms, 6/8 phase partial Fourier, GRAPPA acceleration factor 2, 7 averages) with 20 diffusion directions and a b-value of $2000 \mathrm{~s} / \mathrm{mm}^{2}$. Interspersed were 7 acquisitions with $b=0 \mathrm{~s} / \mathrm{mm}^{2}$.

\section{Segmentation}

FEM head models were created for two subjects. In a first run, different tissue types including WM, GM, CSF, skin and skull were segmented in an automated manner using SimNibs (Windhoff et al., 2013). Special attention was given to tissue interfaces as important physical effects occur there due to charge accumulation and secondary electric fields (Miranda et al., 2003, Thielscher et al., 2011).

The first version of the head models was further refined in a semi-automatic process. This was based on anatomical masks for the vitreous body of the eyes, the surrounding eye regions and the skull (including the nasal cavities) which had been prepared in $\mathrm{MNI}$ space before. The masks for the vitreous bodies of the eyes and the eye regions were hand-drawn based on the T1 MNI template and covered on purpose spatially slightly larger areas. The skull mask was based on the skull reconstructions of the Ella and Duke models of the virtual family (Christ et al., 2010) that had been registered to $\mathrm{MNI}$ space by non-linear warping (FSL FNIRT). It was further improved by manual corrections.

After non-linear registration of the whole-head T1 image onto the MNI template (FSL FNIRT), the masks could be accurately registered onto the individual MRIs. The transformed mask of the vitreous bodies was applied to the fat suppressed T2-image and the final geometries were extracted by histogram-based thresholding. The mask for the surrounding eye regions was applied to the non-fat suppressed T2 and histogrambased thresholding was again used to determine the final geometries. If necessary, the skull mask was manually corrected after transformation. The spongy bone was then segmented by applying the mask to the non-fat suppressed T1 image, followed by histogram-based thresholding. Spurious voxels with high intensities were excluded by means of spatial clustering and applying a threshold for the minimum cluster size. The 
final volume masks were used to create triangle surfaces using the methods described in (Windhoff et al., 2013) and a tetrahedral volume mesh was built. The resolution of tetrahedral elements was enhanced near the electrodes as well as at GM/WM tissue interfaces. This semi-automatic process allows for a time-efficient construction of geometrically accurate head models that include important anatomical features for tDCS simulations. A cut-through image of the FEM head model showing the different tissue volumes is depicted in Figure 1A. Conductivity anisotropy for WM and GM was estimated from the diffusion tensor using a volume normalized approach as described in (Opitz et al., 2011). In short it is assumed that the eigenvectors are the same for the diffusion as well as for the conductivity tensor. The eigenvalues are transferred such that the mean conductivity of the anisotropic case is identical with the isotropic one.

\section{Simulations}

For both subjects the anode was placed directly above the hand area of M1 and the cathode over the contralateral supraorbital region as typically employed in tDCS studies aimed at inducing motor plasticity. The electrode size was set to $7 \mathrm{~cm} \times 5 \mathrm{~cm}\left(35 \mathrm{~cm}^{2}\right)$, which is commonly used in experimental studies. The longer edge of the anode was aligned parallel to the central sulcus such that it approximately covered the precentral gyrus. In order to study the effect of slight changes in electrode placement we moved the anode in steps of $5 \mathrm{~mm}$ anterior and posterior as well as medial and lateral (from $2 \mathrm{~cm}$ to $+2 \mathrm{~cm}$ in both directions). Furthermore, we rotated the anode around its center in 15 degree steps until a complete 180 degree turn was completed as further rotations would result in the same montage again due to symmetry. In total, 28 different electrode montages were simulated for each subject. For an illustration of the simulated montages see Figure 1B. The cathode position was left unchanged across simulations. Electrode thickness for both anode and cathode was $5 \mathrm{~mm}$. 14 additional simulations were performed for the second subject where the electrode was translated anterior-posterior and medial-lateral around a center position that was two centimeter anterior to M1 as the subject exhibited a thinner skull around that region which was of particular interest for our study. 
The following conductivity values were used for simulation unless indicated otherwise: $\sigma_{\text {skin }}=0.25 \mathrm{~S} / \mathrm{m}, \quad \sigma_{\text {compact bone }}=0.008 \mathrm{~S} / \mathrm{m}, \sigma_{\text {spongy bone }}=0.025 \mathrm{~S} / \mathrm{m}$, $\sigma_{C S F}=1.79 \mathrm{~S} / \mathrm{m}, \sigma_{G M}=0.276 \mathrm{~S} / \mathrm{m}, \sigma_{W M}=0.126 \mathrm{~S} / \mathrm{m}, \sigma_{\text {eyeball }}=0.50 \mathrm{~S} / \mathrm{m}, \sigma_{\text {eye region }}=$ $0.25 \mathrm{~S} / \mathrm{m}$ and $\sigma_{\text {electrodes }}=1.79 \mathrm{~S} / \mathrm{m}$. Anisotropic conductivity values were used for WM and GM and all other tissues were treated as isotropic. The electric field was calculated by numerically solving $\vec{E}=-\nabla \varphi$, where $\vec{E}$ is the electric field vector and $\varphi$ the electric potential. The electric potential $\varphi$ was computed using Dirichlet boundary conditions at the electrodes with a fixed potential. $\vec{E}$ was computed by taking the numerical gradient of the electric potential. The potential difference and the electric field values were scaled such that a current of $1 \mathrm{~mA}$ was passing through the electrodes. To identify the effects of spongy bone on the electric field distribution in the brain, the same set of simulations were repeated for the first subject by setting $\sigma_{\text {spongy bone }}=\sigma_{\text {compact bone }}$.

Finally, another 9 simulations were performed to examine the influence of electrode thickness and conductivity. Thickness of the stimulation electrode was varied between 2, 5 and $10 \mathrm{~mm}$ and its conductivity values between 0.2, 2 and $20 \mathrm{~S} / \mathrm{m}$. Thus, a large range of possible combinations that could in practice have an influence on the electrode characteristics were covered.

\section{Data Analysis}

For each head model the following measures were computed: Skull thickness, CSF thickness and sulcal depth. Skull thickness was defined as the distance between the outer surface of the compact bone and the CSF-bone interface. A skull thickness value was computed for each node of the skull surface. In addition, a skull thickness value was attributed to each node of the GM surface by taking the value of the closest node of the skull surface. The aim was to capture the influence of the skull thickness on the electric field in the underlying brain region. The CSF thickness was computed by taking the distance between the GM and the CSF surface for each node on the GM surface, respectively. Sulcal depth was determined by using the values computed by Freesurfer 
(http://surfer.nmr.mgh.harvard.edu/) applying a nearest-neighbor interpolation. Sulcal depth values were normalized to their mean.

Furthermore, for each simulation the distances of the gray matter positions to the electrode edges were assessed. This was done in two steps. First, the electrode edge on the scalp was projected onto the GM surface. Second, for each node on the GM the Euclidian distance to the nearest node of the projected electrode edge on the brain was computed.

The relationship between the electric field in the vicinity of the stimulating electrode and the anatomical factors were studied using multiple linear regression. The analysis was restricted to a region of interest $(\mathrm{ROI})$ centered on the motor cortex and including the brain areas that were directly underneath the stimulating electrode for at least one of the simulated positions plus an additional $\sim 2 \mathrm{~cm}$ of surrounding GM. By that, we ensured that the brain regions on which the stimulation electrode exerted strong effects were included into the analysis. The ROls for both subjects are shown in Figure 1C. The covered region for Subject 2 was slightly larger in the anterior direction, reflecting the fact that we included more anterior anode positions in that subject. The regions underneath the reference electrode or the electric field in remote brain areas were not in the focus of this study.

The absolute electric field value at each node on the GM surface in the ROI was regressed against a linear combination of skull thickness, sulcal depth, CSF thickness and distance to the electrode edge. As CSF thickness and sulcal depth were naturally correlated, we regressed out the effect of sulcal depth on CSF thickness and used the residuals of the CSF thickness - sulcal depth regression instead of the original CSF thickness in the regression model. To determine whether the amount of explained variance exhibited a statistically significant increase with an increasing number of explanatory factors, we used a hierarchical testing approach and assessed sequential F-tests when adding one additional explanatory variable at each step. Regression models and F-tests were run separately for each simulated position. F-values were computed and compared to their critical value of the 95-th percentile of the F- 
distribution. The mean and standard deviation of the amount of explained variance $r^{2}$ was computed over all positions and assessed for reporting.

Finally, we aimed at evaluating whether certain brain areas were especially prone to being stimulated. To do so, we first determined the GM nodes that exhibited electric field strengths greater than $80 \%$ of the maximum of the electric field occurring on the GM surface. This was done separately for each of the positions of the stimulating electrode. Subsequently, an "index of activation likelihood" was computed for each GM node by determining the number of times in which its electric field exceeded the $80 \%$ threshold across all positions.

\section{Results}

As a first step, the influence of anatomical factors on the electric field distribution in the cortex can be exemplarily demonstrated by focusing on the effects of the skull thickness and of the distance to the electrode edges (Fig. 2A). Generally, the electric field (and, likewise, the current flow) is strongest underneath the electrode edges. However, this effect is clearly modulated by the skull thickness: Thinner skull parts (indicated by the circles in Fig. 2A) exhibit the highest field strengths. This in turn causes increased field strengths in the underlying GM parts (Fig. 2B). This effect is stable and largely independent of explicitly modeling the spongy bone (Fig. 2B left panel) or setting the conductivity of spongy bone to compact bone (Fig. 2B right).

In general, spongy bone can be mostly found in regions where the skull is thick. As spongy bone is better conducting than compact bone, not taking the spongy bone into account will therefore further increase the resistance of thick skull regions. By that, the current flow will be even more channeled towards thin skull parts (Fig. 2C). However, the overall effect on the electric field in GM seems moderate and in the range of $\sim 20 \%$. Visual inspection reveals that the effects of spongy bone as depicted here for a specific electrode position are generally observed across all simulated positions (data not shown). 
A more complete picture arises when taking sulcal depth and thickness of the overlying CSF layer as additional factors into account (Fig. 3). Very high field strengths (indicated by the black circle in Fig. 3E) occur in a GM region which is at a gyral crown (i.e., has a low sulcal depth; Fig 3B), has a thin overlying skull (Fig. 3A), a thin overlying CSF layer (Fig. 3C) and is directly below the edge of the electrode (Fig. 3D). Regions exhibiting a high but not maximal field strength show a similar combination of factors yet to a lesser extent. For example, the region indicated by the red circle in Figure 3E is not fully underneath the electrode edge, but still shows an "optimal" combination of the other three factors. For regions of weak electric field strength, a reverse relation to the underlying factors can be observed.

The relationship between the electric field in GM and the discussed factors can be more systematically visualized by means of two-dimensional histograms. For that purpose, the value pairs for the field strength and the anatomical features from all nodes within the GM ROIs are binned into a two dimensional grid and the number of nodes falling within each bin of the grid is determined and coded as a frequency of occurrence. The histograms for Subject 1 for one exemplary electrode position are shown in Figure 4. For example, Figure $4 \mathrm{~A}$ depicts the dependence of the electric field on the skull thickness, showing higher electric field strength with lower skull thickness. The relationship between the field strength and the other three factors - CSF thickness, sulcal depth and distance to the electrode edge - show similar patterns (Fig. 4B-D).

The total explained variance of the regression model across all simulated electrode positions is $r^{2}=0.48 \pm 0.04$ for Subject 1 . For Subject 2 the amount of explained variance is reduced to $r^{2}=0.30 \pm 0.02$ for the first set of simulations over the motor regions. Visual inspection of the results revealed that the underlying reason is a less variable distribution of skull and CSF thickness values in the region underneath the tested electrode positions. When testing 14 additional, more anterior electrode positions in Subject 2, the explained variance increases to $r^{2}=0.51 \pm 0.06$. Sequential F-tests showed that the increase in explained variance by adding explanatory factors is statistically significant at $p=0.05$ for all factors for all but one of the 70 simulated 
positions. This demonstrates that the explored features exert a robust and significant influence on the electric field strength in the cortex.

Further, we were interested to explore whether the position of the maximally stimulated cortical area changes smoothly with the position of the stimulating electrode, despite the observed influence of anatomical factors. For each simulated position, the GM areas that exhibit electric field strength greater than $80 \%$ of the maximal field strength were determined. An "index of activation likelihood" was then defined that indicates how often a cortical area exceeds the threshold across all simulated electrode positions. A homogeneous distribution of low likelihood values across the cortex indicates that the maximally stimulated area is mainly determined by the electrode position. On the other hand, an activation index of 1 means that this brain region was stimulated stronger than $80 \%$ of the maximum in all simulations, irrespective of the actual electrode placement. Please note that the threshold of $80 \%$ is an arbitrarily chosen value. However, setting the threshold to $70 \%$ or $90 \%$ reveals similar results (data not shown). Figure $5 \mathrm{~A}$ shows the results for Subject 1 . The highest likelihood of activation occurs in premotor regions for both electrode translations (left panel) and rotations (right panel). A similar pattern was visible for Subject 2, again for both translations (Fig. 5B left panel) and rotations (Fig. 5B right panel). This suggests that anatomical factors are highly determinant of the positions on which the electric field exerts its strongest influence despite the movement of the electrode over an area of $4 \times 4 \mathrm{~cm}$ on the scalp.

The final set of simulations was aimed at ruling out that the simulated electrode characteristics might have strongly influenced the findings reported so far. However, for a wide range of thicknesses and conductivities, the electric field distribution on the scalp stays widely constant (Fig. 6). While the overall influence is small, a few effects are still observable: For low electrode conductivities the electric field is more smeared out compared to the high conductivities for which the field was more confined. 


\section{Discussion}

The spatial distribution of the electric field that is injected in the cortex during tDCS is a primary determinant of the resulting neural and behavioral effects. Here, we show that the field strength depends on several anatomical factors - the thickness and composition of the overlying skull, the thickness of the CSF layer between the cortex and skull and the sulcal depth. In addition, it is scaled by the distance of a cortical position to the closest electrode edge.

The strong influence of the skull on the field in the brain results from the fact that it is the least conductive medium in the human head. Currents pass approximately radially through it when flowing from the electrode to the brain and the radial conductivity of thin parts of the skull is higher compared to surrounding skull regions. This establishes a preferred path for current flow that results in higher field strengths in thin skull areas and in underlying brain tissue. We demonstrate that this effect is largely independent of spongy bone being explicitly modeled or not. Setting the conductivity of spongy bone to that of compact bone results in a modulation of the electric field in the cortex in the range of up to $20 \%$. Spongy bone is mainly found in the thicker regions of the skull and enhances the radial conductivity of these regions. By that, the current flow tends to be more evenly distributed across skull regions of different thicknesses when explicitly modeling the spongy bone compartments. However, the overall effect is small and current flow still occurs preferentially through thin skull regions. As a consequence, brain areas under thinner skull regions like the temporal bone will be more readily stimulated by tDCS than areas under thick skull regions. This effect might be very important for dosage considerations for tDCS. Our results tie in nicely with prior findings on role of the net radial conductivity of the skull in influencing current flow in a more simplified model (Rampersad et al., 2013) and on the impact of skull openings on the field in underlying brain areas (Datta et al., 2010, Mekonnen et al., 2012). In a similar vein, skull conductivity and sutures have also strong effect on the accuracy of source localization in EEG (Dannhauer et al., 2011). The detailed skull conductivity has smaller

influences on TMS and MEG (Lew et al., 2013) as the induced current flow and the measured sources, respectively, are mainly tangentially orientated in these cases. 
The general dependence of the electric field on the sulcal depth is an expected finding since current spread results in lower current densities and hence electric field strengths in regions further down in the sulci (see also Wagner et al., 2013). In Miranda et al. (2013) peaks of the normal electric field component have been reported to occur in deeper sulcal regions. It should be noted that this finding only refers to localized field maxima, while the tangential field (which, according to their report, mainly occurs in superficial brain areas) is on average still stronger than the normal component. In addition, the peak values are in about the same order of magnitude $(0.2-0.3 \mathrm{mV} / \mathrm{mm})$ as we find with our model in deeper sulcal regions. However, the strongly enhanced ( $1 \mathrm{mV} / \mathrm{mm}$ ) superficial electric fields caused by anatomical factors such as skull thinning diminishes the relative contribution of these electric fields in deeper brain areas. Interestingly, even when factoring the effect of sulcal depth out, the thickness of the CSF layer still has a significant impact on the field strength in the underlying cortex region. A thin layer of CSF increases the field in the underlying brain region: CSF is the domain in the head with the highest conductivity and is thus a preferential pathway for the injected currents. With only a thin CSF layer over the brain, less current is carried away before entering the GM. A related effect of CSF has been demonstrated for EEG where the amplitudes of signals recorded over the occipital cortices were altered due to more or less CSF covering it, depending on the subjects body positions (prone or supine; Rice et al., 2013). Similarly, in case of TMS, it is suggested that gyri closer to the skull tend to experience higher field strength as they are within the pathway of strong currents flowing tangentially in superficial CSF layers (Bijsterbosch et al., 2012).

In addition to individual anatomical factors, the distance of a cortical region to the nearest edge of the electrode influences the field strength. Consistent with the results of previous studies (Miranda et al., 2013), this effect is most pronounced in the skin and skull but can be also observed in underlying tissues. Depending on the simulated participant, the unique combination of individual anatomical features can result in a highly specific electric field distribution in the brain. However, we demonstrate here in two head models that up to $50 \%$ of the variance of the field in the most strongly affected cortical areas can be explained by a linear regression model relying on four comparatively simple factors. 
Combinations of anatomical factors can occur which result in a very inhomogeneous field distribution in the cortex. Specifically, our results show that brain areas in the proximity to both thin CSF and skull layers and close to an electrode edge can experience high field strength. As a result, when determining which brain areas are most strongly stimulated over all electrode positions, only one or two single brain regions pop out. In both head models, these regions do not coincide with the primary motor cortices but with more anterior areas. Importantly, they are also the most stimulated areas when the electrode was placed centrally above M1, as would be the case in a practical experiment. Our findings are robust to variations in electrode thickness and conductivity as the latter had only a minor influence on the electric field. This further indicates that the choice of the electrode type (e.g. saline soaked sponge electrodes or rubber electrodes covered by a conducting gel) can thus be guided by practical considerations in experiments.

Adjusting the electrode position is the main measure taken in practical tDCS experiments for targeting a certain brain structure. Our results obtained in two head models indicate that the effectiveness of this approach can be strongly limited by anatomical factors and that it can very difficult to steer a significant amount of current to the selected target region. As anatomy exhibits interindividual variability, this further means that the same montage can stimulate very different brain regions across different subjects. Thus, variability in anatomy very likely contributes strongly to the interindividual variability in the physiological and behavioral tDCS effects. Future studies using subject-specific FEM models for tDCS should investigate if physiological different effects between subjects can be explained by computational biophysical models as has already been demonstrated in the case of TMS (Thielscher and Wichmann, 2009, Opitz et al., 2013). The impact of anatomical specificities could possibly be accounted for by using multielectrode targeting approaches (Dmochowski et al., 2011) which would adjust stimulating currents such that a maximal electric field could be reached at the target area despite the hindering anatomical factors. Our results indicate that the accuracy of those approaches might benefit strongly from using subject-specific rather than template FEM models. 
In conclusion we show how various anatomical factors of the human head influence the electric field distribution in the brain. Approximately $50 \%$ of the variation of the field in a $\mathrm{ROI}$ underneath the stimulation electrode can be explained using four simple measures to capture the thickness of the skull and CSF layer, the sulcal depth and the distance to the closest electrode edge. Furthermore, we describe the influence of the electrode position and its interaction with anatomical features on stimulated brain regions. We demonstrate that individual anatomy has a strong influence on the electric field distribution that can largely determine the stimulated area irrespective of the exact electrode position. The knowledge of the influence of the described anatomical factors can be important for the interpretation of tDCS effects as well as for the design of optimized stimulation protocols. While we investigated the effects of individual anatomy on the electric field for transcranial direct current stimulation, they also apply for other forms of transcranial electrical stimulation, like transcranial alternating current stimulation (tACS) or transcranial random noise stimulation (tRNS).

\section{References}

Bijsterbosch JD, Barker AT, Lee KH, Woodruff PW (2012) Where does transcranial magnetic stimulation (TMS) stimulate? Modelling of induced field maps for some common cortical and cerebellar targets. Medical \& biological engineering \& computing 50:671-681.

Bikson M, Inoue M, Akiyama H, Deans JK, Fox JE, Miyakawa H, Jefferys JG (2004) Effects of uniform extracellular DC electric fields on excitability in rat hippocampal slices in vitro. The Journal of physiology 557:175-190.

Christ A, Kainz W, Hahn EG, Honegger K, Zefferer M, Neufeld E, Rascher W, Janka R, Bautz W, Chen J, Kiefer B, Schmitt P, Hollenbach HP, Shen J, Oberle M, Szczerba D, Kam A, Guag JW, Kuster N (2010) The Virtual Family--development of surface-based anatomical models of two adults and two children for dosimetric simulations. Physics in medicine and biology 55:N23-38.

Dannhauer M, Lanfer B, Wolters CH, Knosche TR (2011) Modeling of the human skull in EEG source analysis. Hum Brain Mapp 32:1383-1399.

Datta A, Bansal V, Diaz J, Patel J, Reato D, Bikson M (2009) Gyri-precise head model of transcranial direct current stimulation: improved spatial focality using a ring electrode versus conventional rectangular pad. Brain stimulation 2:201-207, 207 e201.

Datta A, Bikson M, Fregni F (2010) Transcranial direct current stimulation in patients with skull defects and skull plates: high-resolution computational FEM study of factors altering cortical current flow. Neuroimage 52:1268-1278. 
Datta A, Elwassif M, Battaglia F, Bikson M (2008) Transcranial current stimulation focality using disc and ring electrode configurations: FEM analysis. Journal of neural engineering 5:163-174.

Dmochowski JP, Datta A, Bikson M, Su Y, Parra LC (2011) Optimized multi-electrode stimulation increases focality and intensity at target. Journal of neural engineering 8:046011.

Lew S, Sliva DD, Choe MS, Grant PE, Okada Y, Wolters CH, Hamalainen MS (2013) Effects of sutures and fontanels on MEG and EEG source analysis in a realistic infant head model. Neurolmage 76:282-293.

Mekonnen A, Salvador R, Ruffini G, Miranda PC (2012) The relationship between transcranial current stimulation electrode montages and the effect of the skull orbital openings. Conference proceedings : Annual International Conference of the IEEE Engineering in Medicine and Biology Society IEEE Engineering in Medicine and Biology Society Conference 2012:831-834.

Miranda PC, Hallett M, Basser PJ (2003) The electric field induced in the brain by magnetic stimulation: a 3-D finite-element analysis of the effect of tissue heterogeneity and anisotropy. IEEE transactions on bio-medical engineering 50:1074-1085.

Miranda PC, Lomarev M, Hallett M (2006) Modeling the current distribution during transcranial direct current stimulation. Clinical neurophysiology : official journal of the International Federation of Clinical Neurophysiology 117:1623-1629.

Miranda PC, Mekonnen A, Salvador R, Ruffini G (2013) The electric field in the cortex during transcranial current stimulation. Neuroimage 70:48-58.

Neuling T, Wagner S, Wolters CH, Zaehle T, Herrmann CS (2012) Finite-Element Model Predicts Current Density Distribution for Clinical Applications of tDCS and tACS. Frontiers in psychiatry 3:83.

Nitsche MA, Paulus W (2000) Excitability changes induced in the human motor cortex by weak transcranial direct current stimulation. The Journal of physiology 527 Pt 3:633-639.

Nitsche MA, Paulus W (2001) Sustained excitability elevations induced by transcranial DC motor cortex stimulation in humans. Neurology 57:1899-1901.

Opitz A, Legon W, Rowlands A, Bickel WK, Paulus W, Tyler WJ (2013) Physiological observations validate finite element models for estimating subject-specific electric field distributions induced by transcranial magnetic stimulation of the human motor cortex. Neurolmage.

Opitz A, Windhoff M, Heidemann RM, Turner R, Thielscher A (2011) How the brain tissue shapes the electric field induced by transcranial magnetic stimulation. Neurolmage 58:849-859.

Radman T, Ramos RL, Brumberg JC, Bikson M (2009) Role of cortical cell type and morphology in subthreshold and suprathreshold uniform electric field stimulation in vitro. Brain stimulation 2:215-228, 228 e211-213.

Radman T, Su Y, An JH, Parra LC, Bikson M (2007) Spike timing amplifies the effect of electric fields on neurons: implications for endogenous field effects. The Journal of neuroscience : the official journal of the Society for Neuroscience 27:3030-3036.

Rampersad SM, Stegeman DF, Oostendorp TF (2013) Single-layer skull approximations perform well in transcranial direct current stimulation modeling. IEEE transactions on neural systems and rehabilitation engineering : a publication of the IEEE Engineering in Medicine and Biology Society 21:346-353.

Rice JK, Rorden C, Little JS, Parra LC (2013) Subject position affects EEG magnitudes. Neurolmage 64:476-484.

Sadleir RJ, Vannorsdall TD, Schretlen DJ, Gordon B (2010) Transcranial direct current stimulation (tDCS) in a realistic head model. Neuroimage 51:1310-1318.

Suh HS, Lee WH, Kim TS (2012) Influence of anisotropic conductivity in the skull and white matter on transcranial direct current stimulation via an anatomically realistic finite element head model. Physics in medicine and biology 57:6961-6980. 
Thielscher A, Opitz A, Windhoff M (2011) Impact of the gyral geometry on the electric field induced by transcranial magnetic stimulation. Neurolmage 54:234-243.

Thielscher A, Wichmann FA (2009) Determining the cortical target of transcranial magnetic stimulation. Neurolmage 47:1319-1330.

Wagner S, Rampersad SM, Aydin U, Vorwerk J, Oostendorp TF, Neuling T, Herrmann CS, Stegeman DF, Wolters CH (2013) Investigation of tDCS volume conduction effects in a highly realistic head model. Journal of neural engineering 11:016002.

Windhoff M, Opitz A, Thielscher A (2013) Electric field calculations in brain stimulation based on finite elements: an optimized processing pipeline for the generation and usage of accurate individual head models. Hum Brain Mapp 34:923-935.

\section{Figure Captions:}

Figure 1: A) Cut-through image of the FEM head model of Subject 1. Shown are the different tissue types and their composing tetrahedral elements. The skull is composed of three layers: Two layers of compact bone (light gray) are encompassing the spongy bone (yellow). Element resolution is enhanced at tissues underneath and next to the electrodes. B) Illustration of the simulated montages. While the reference electrode (blue) over the contralateral forehead was kept constant, the position of the stimulation electrode (green) over the left motor cortex was systematically varied. The electrode was moved $2 \mathrm{~cm}$ anterior and posterior as well as medial and lateral in $5 \mathrm{~mm}$ steps. Furthermore, the electrode was rotated in 15 degree steps around the central position. C) Brain regions of interest. Shown are the regions of interest (blue) on the brain where the electric field was evaluated for the regression model. The ROIs comprised motor regions as well as neighboring gyri to cover all potential regions with high electric field strengths. For Subject 2 the ROI was more extended in the anterior direction, as for this subject additional simulations were performed with more anterior electrode positions.

Figure 2: Effect of skull thickness. A) The electric field on the skull is depicted for one representative electrode position (left panel; the electrode outline is indicated as white line). Regions of strongest electric field occur at the electrode edge especially in more lateral parts of the skull (black and red circles). These regions of enhanced electric field strength coincide with regions of thin skull (right panel). B) Areas underlying thin skull experience higher electric field strength than surrounding brain tissue (black and red 
circles). This effect is independent of the inclusion of spongy bone in the model. Both the models distinguishing between spongy and compact bone (left panel) or not (right panel) show the same qualitative behavior. C) Relative difference between the electric fields on the cortex for the models without versus with spongy bone (left panel). The origin of the observed differences can be understood by relating them to the anatomy of the overlying skull regions (right panel). The field strength in gray and white matter is shown in a coronal slice underneath the stimulation electrode. In addition, the surrounding CSF, skull and skin compartments of the head model and the electrode are depicted. When not distinguishing between spongy (yellow tetrahedra) and compact (beige tetrahedra) bone, the conductivity of thicker parts of the skull is overestimated. This results in too high electric field estimates in the brain areas underlying thin parts of skull, as indicated by the red arrows.

Figure 3: Example demonstrating the effect of several factors on the electric field in the cortex. For visualization, the factors are projected on the inflated brain surface and shown together with the electric field. A) Local skull thinning (black circle) correlates with strong electric fields. B) Regions with low sulcal depth (at the gyral crown) are prone to high electric fields (black and red circles). C) Thin CSF layers (black and red circles) correspond with high electric fields. D) Regions at or near the electrode edge (black and red circles) have high electric fields. E) The electric field distribution exhibits various hotspots, depending on several anatomical factors. The two main hotspots are exemplarily marked (black and red circles).

Figure 4: Quantitative relationship of anatomical features and electrode position with the electric field strength in the brain. Shown are 2D histograms and the regression lines (gray) between the electric field and the explanatory variables for all four factors in the regression model. A) High electric field strength is related with lower skull thickness. B) Increased electric field strength is observed for regions with a thin overlying CSF layer. C) Regions with a low sulcal depth (near or at a gyral crown) have higher field strength than regions with high sulcal depths (at the sulcal fundus). D) Electric field is strongest in regions underneath the electrode edge and drops off with increasing distance. 
Figure 5: The maximally stimulated brain areas are partly pre-determined by anatomical factors. Shown is the index of activation likelihood which indicates how often a brain region is maximally stimulated over all electrode positions. A) Activation for Subject 1 for both electrode translation (left panel) and rotations (right panel). B) Activation for Subject 1 for both electrode translation (left panel) and rotations (right panel). Note that for both subjects the maximally stimulated area is consistently anterior to the primary motor cortex.

Figure 6: Effect of electrode thickness and conductivity. For each simulation, the field strengths is normalized to the individual maximum on the scalp. With increasing electrode conductivity regions of high electric field strength become spatially more confined. 
A

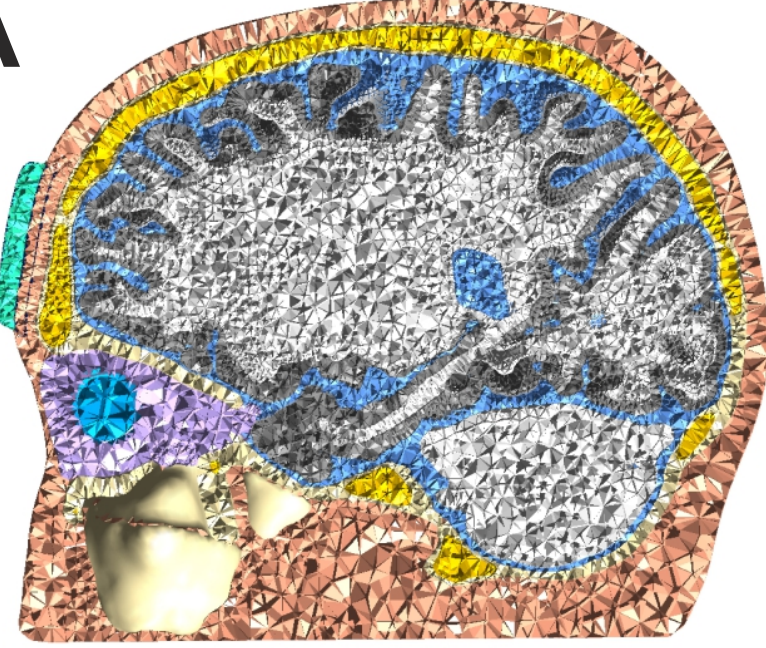

C

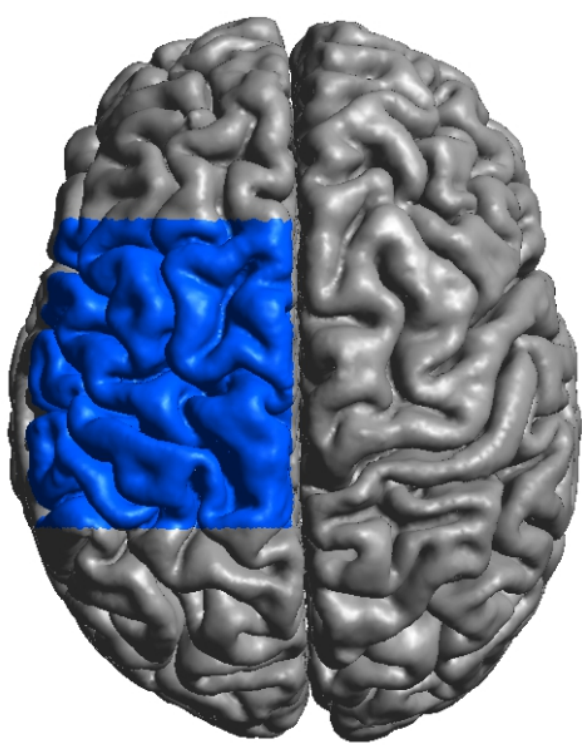

Subject 1
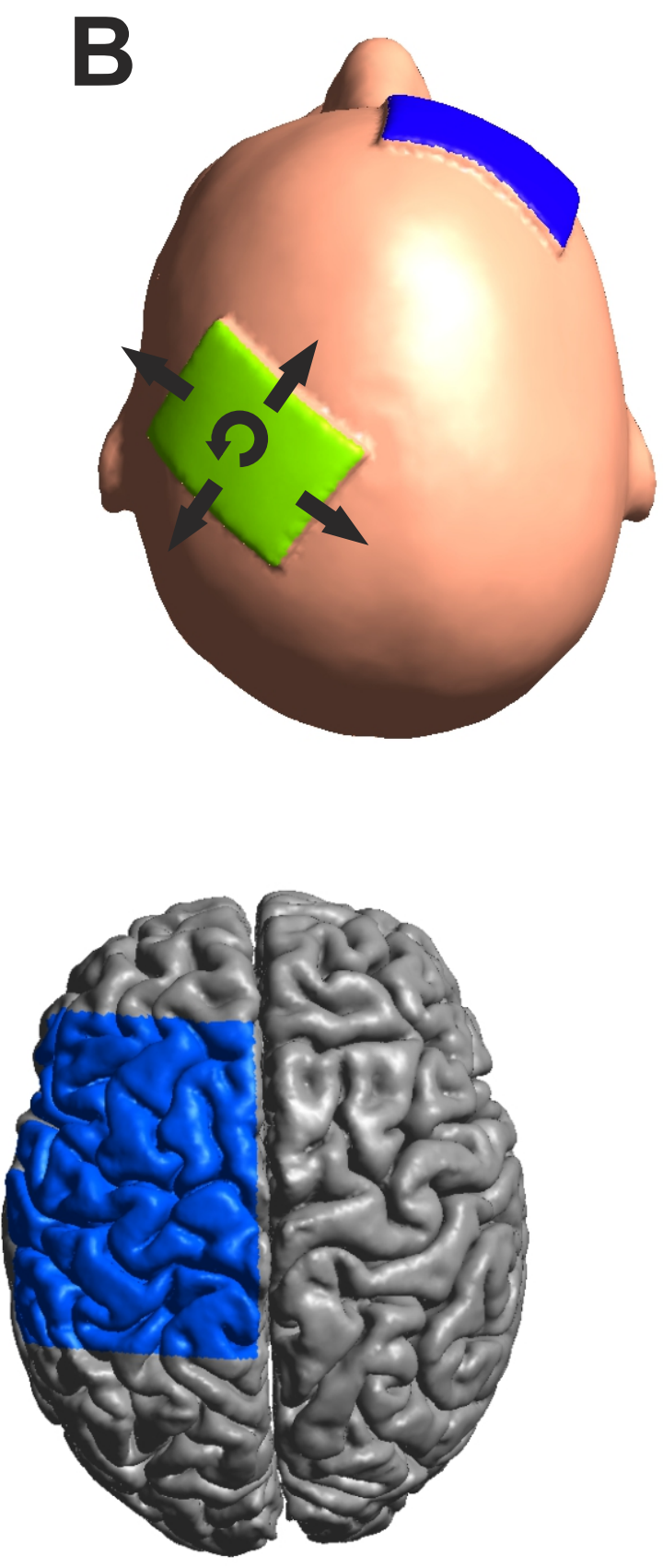

Subject 2 


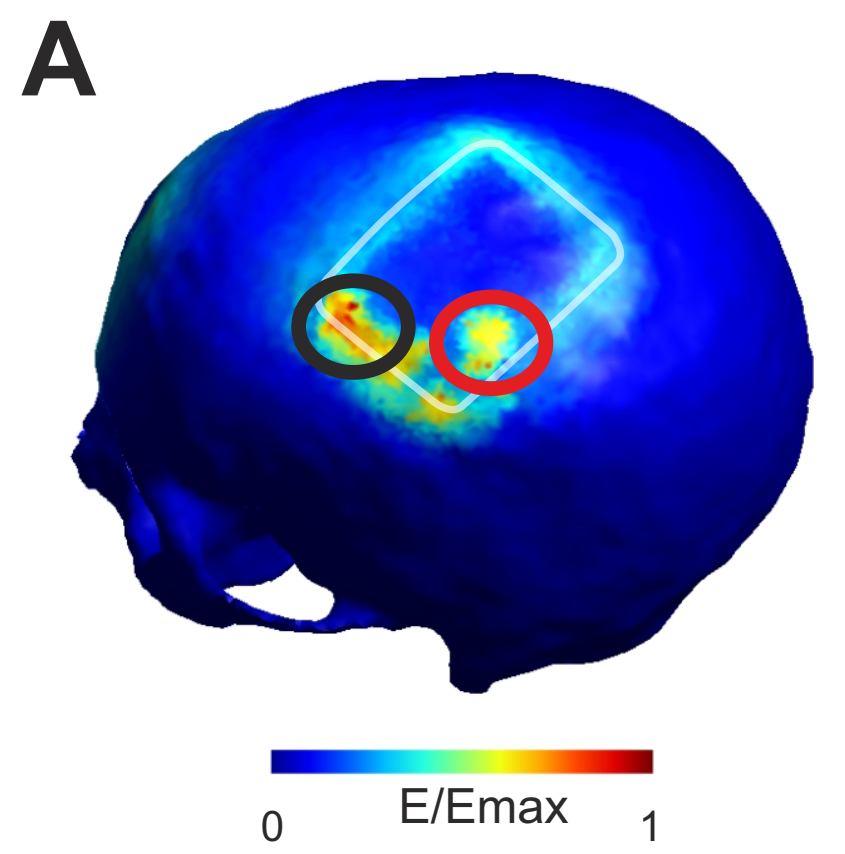

\section{skull thickness}
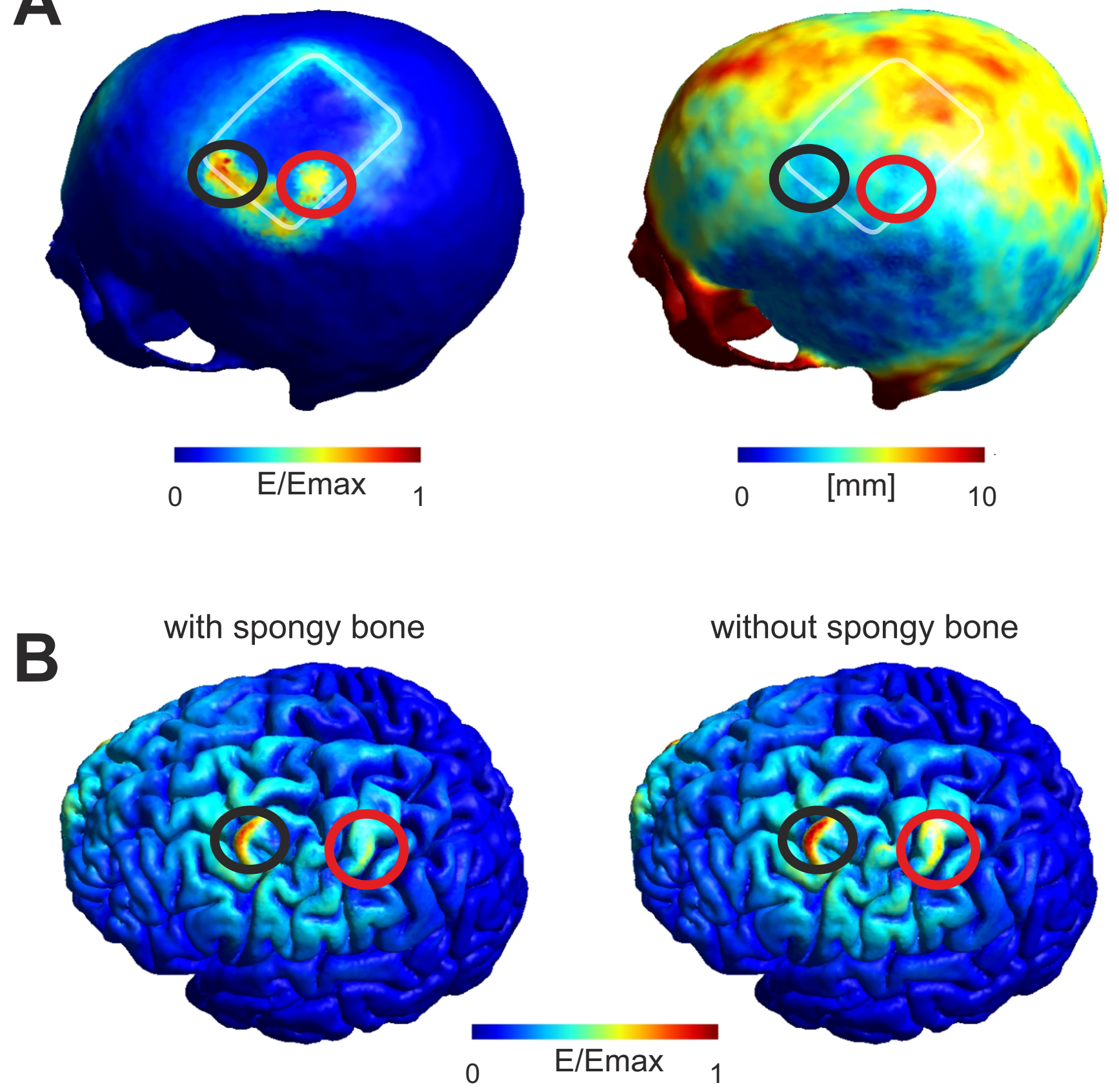

without vs with spongy bone

C

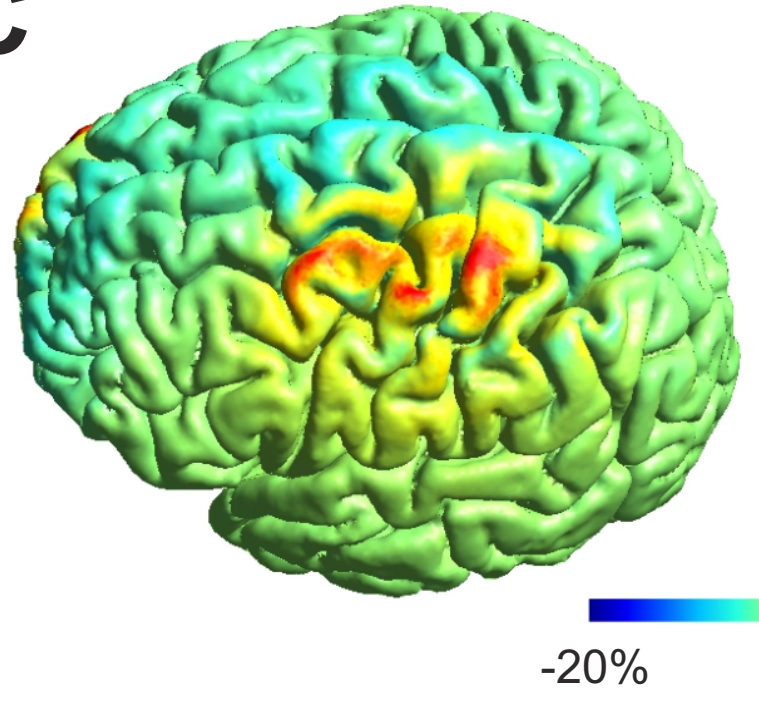

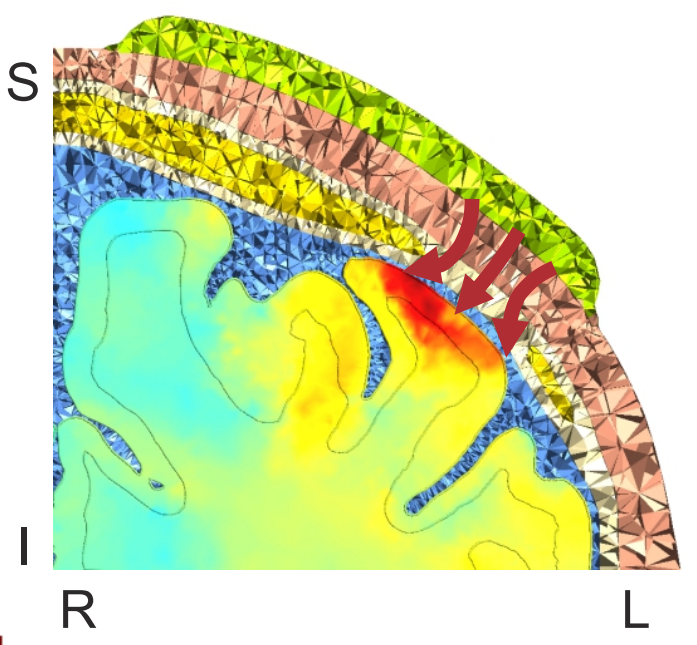



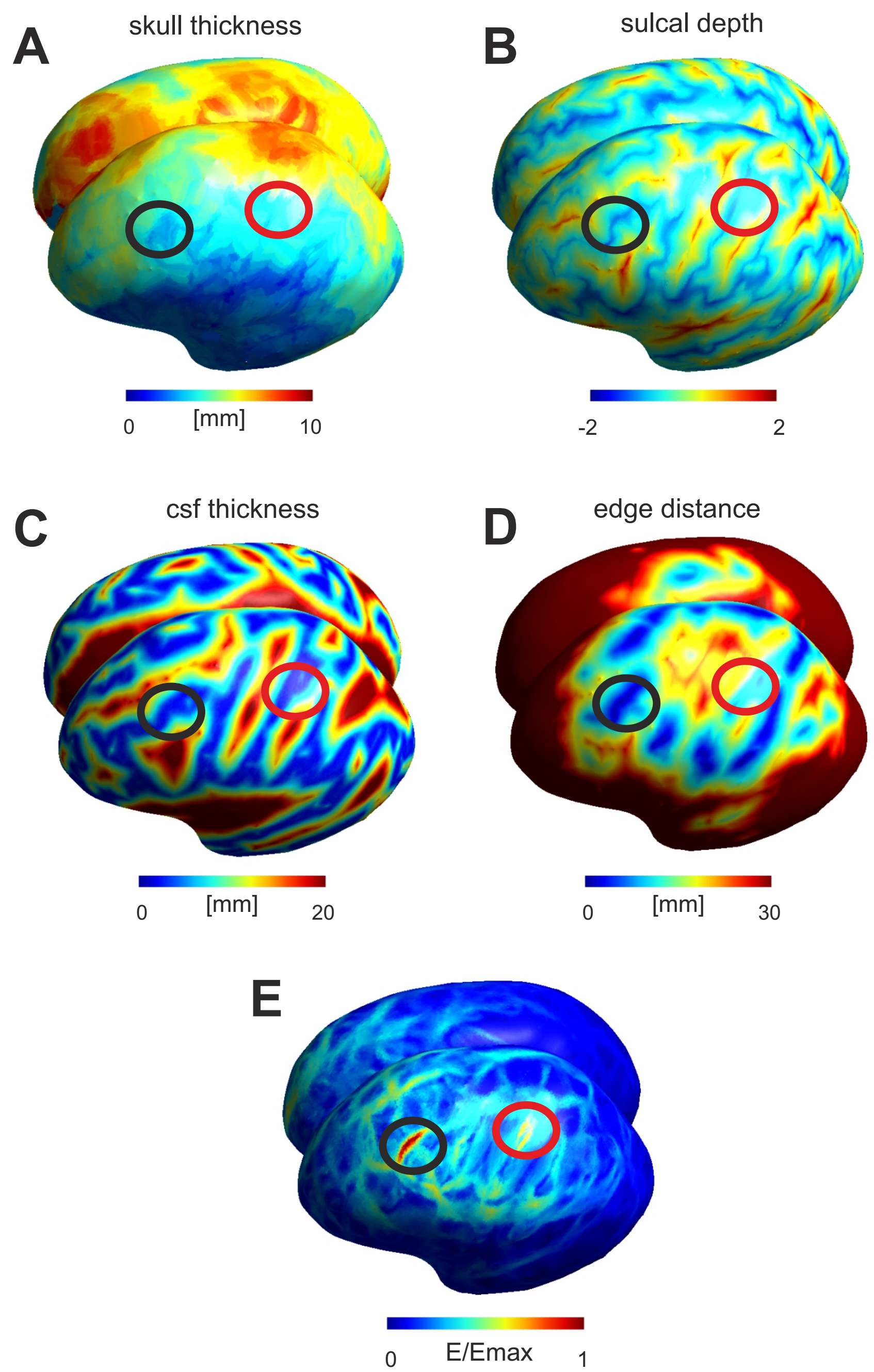

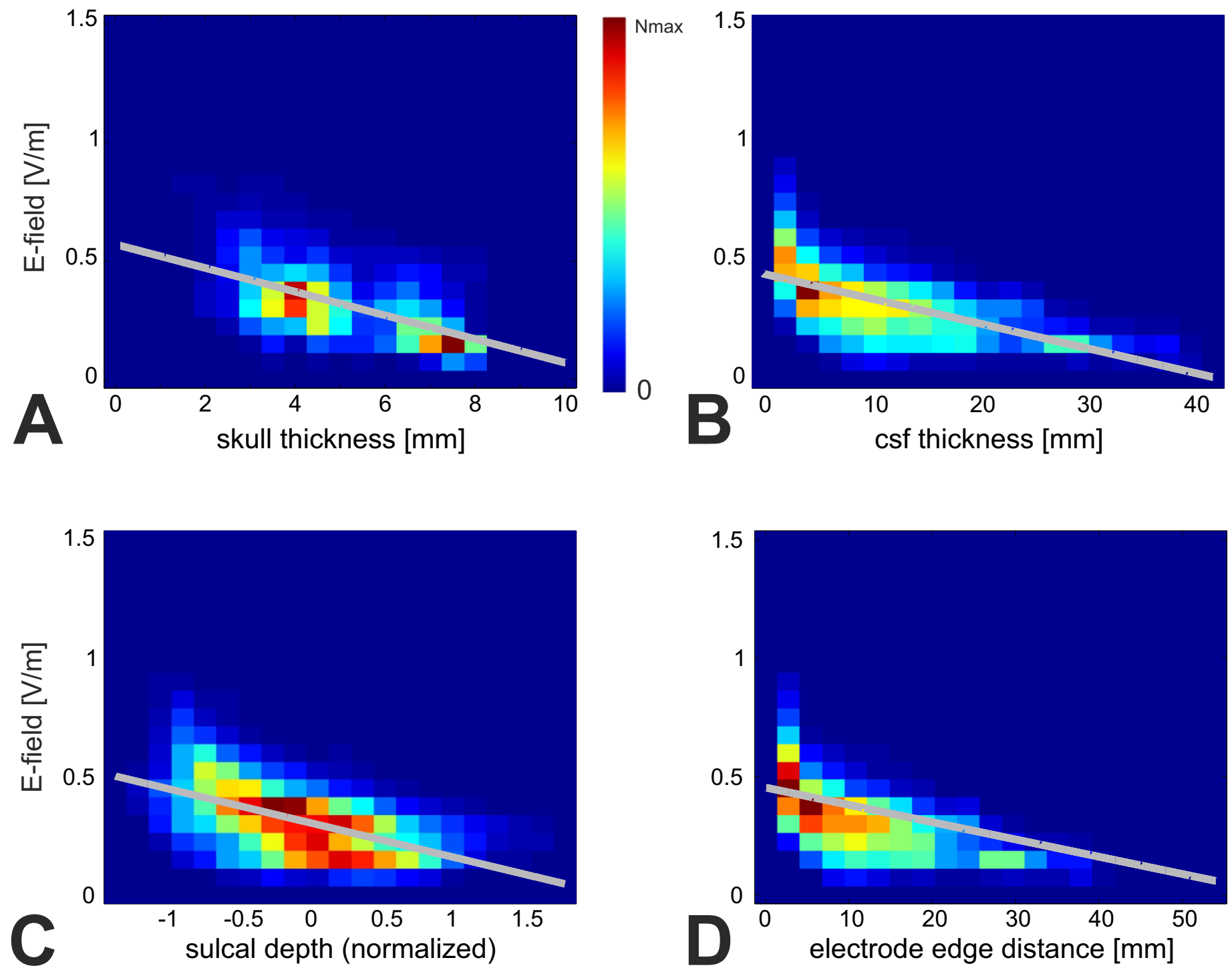
A

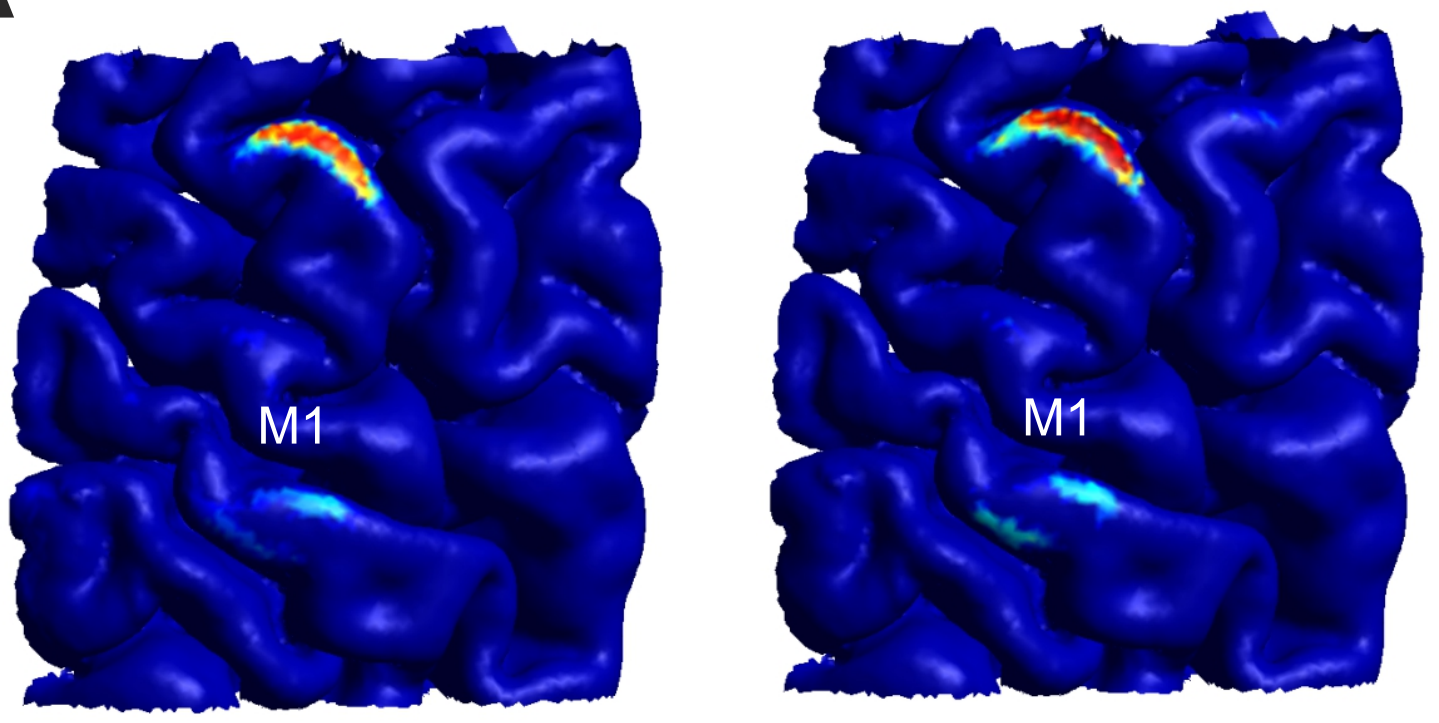

index of activation likelihood

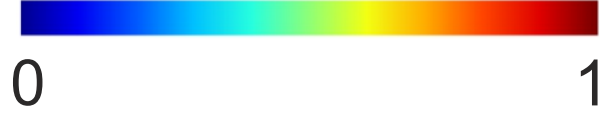

B
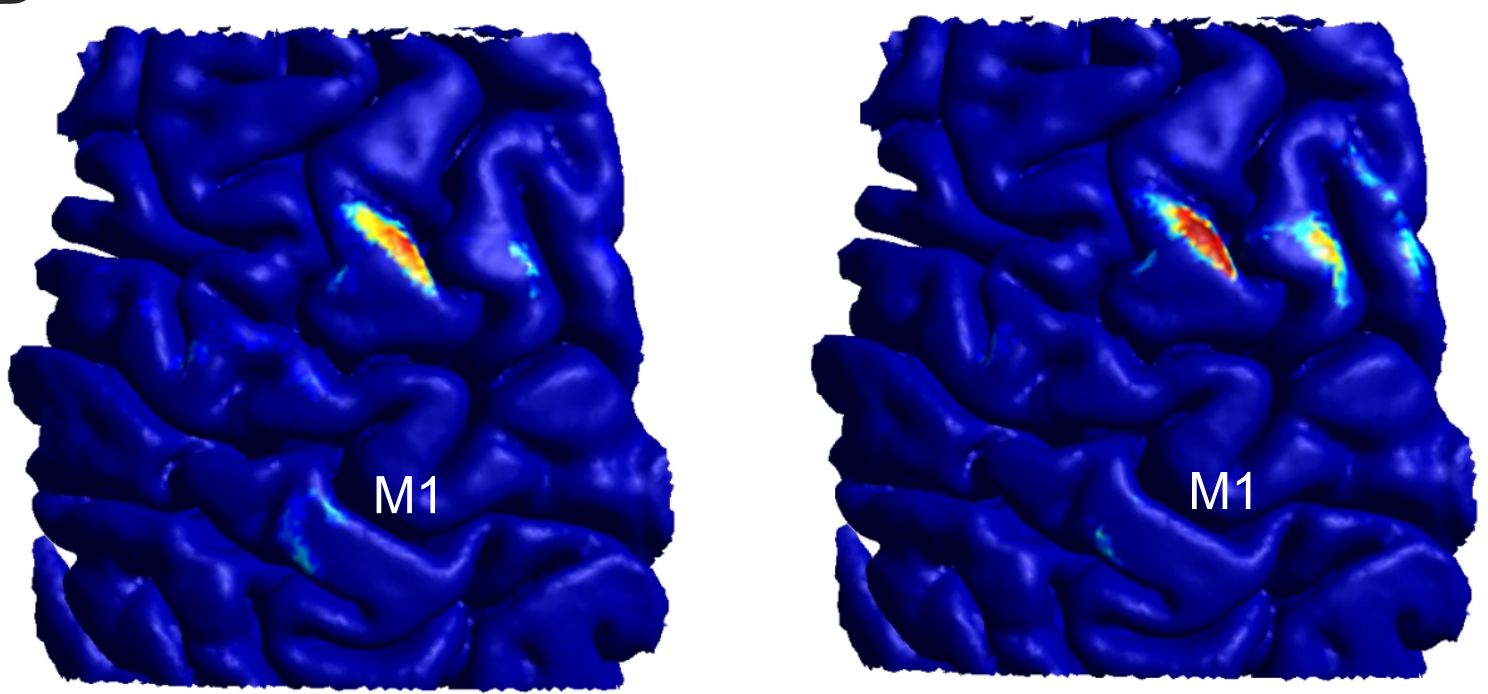


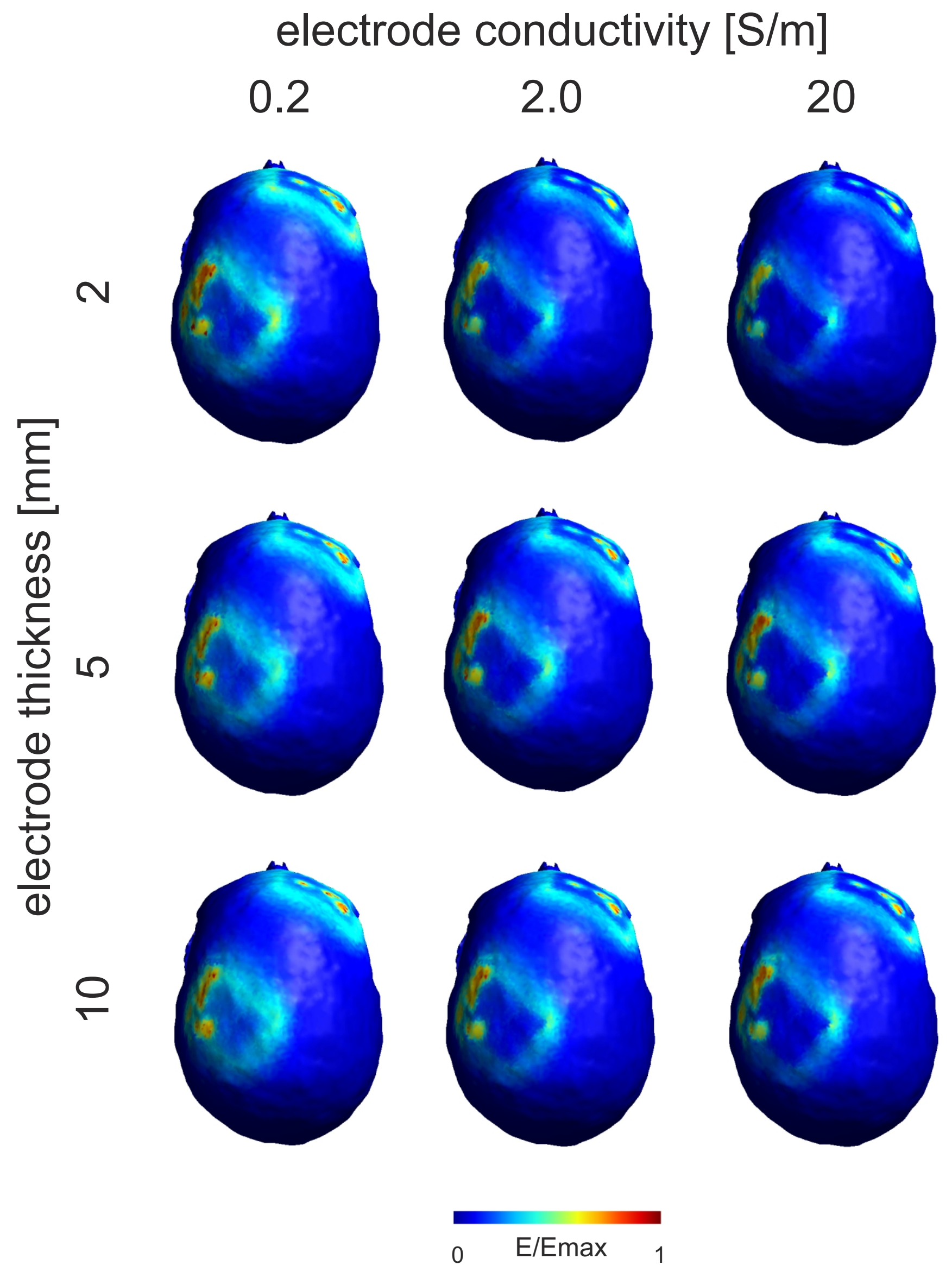




\section{Conclusion}

In this thesis, new computational methods for non-invasive brain stimulation were developed and validated with experimental data. For transcranial magnetic stimulation we found that individual FEM models are able to partly account for the variance in motor evoked potentials and are able to reliably predict stimulation areas in the brain. We further validated the models with direct electrical stimulation measurements in tumor patients. Here we found a good overlap between TMS and DES stimulation areas. We thus conclude that individual electric field modelling is able to more precisely determine stimulated areas in the brain. In the future, these models can help to improve presurgical mapping of eloquent areas in the brain. Also in healthy subjects, targeting accuracy with TMS can be improved, especially in combination with neuroimaging, and might lead to novel stimulation protocols. With increasing automation in the FEM model generation and computational resources, a more widespread use of realistic field calculation in TMS can be reached in the near future.

For transcranial direct current stimulation we found a strong influence of anatomical factors on the electric field distribution. Especially, local skull thinnings were found to be preferential pathways for electric current flow. This resulted in hotspots in the brain which were relatively independent from the exact electrode montage showing that anatomy can partially override the effect of electrode placement. However, the effects of anatomical properties of the head on the electric field distribution in the brain can be accounted for in a regression model. The influence of different components can help for an overall better understanding of the underlying biophysics in tDCS. The strong anatomical dependence might cause practical problems to target chosen brain structures as they might be badly positioned to be stimulated. Here, it can be explored in the future if more refined stimulation strategies like multi electrode systems can overcome existing anatomical constraints in transcranial direct current stimulation. Also, experimental studies linking physiological or behavioral measures with computational predictions are needed to evaluate the usefulness of individual realistic models for tDCS. 


\section{Bibliography}

Amassian VE, Eberle L, Maccabee PJ, Cracco RQ (1992) Modelling magnetic coil excitation of human cerebral cortex with a peripheral nerve immersed in a brain-shaped volume conductor: the significance of fiber bending in excitation. Electroencephalogr Clin Neurophysiol 85:291-301.

Anastassiou CA, Perin R, Markram H, Koch C (2011) Ephaptic coupling of cortical neurons. Nature neuroscience 14:217-223.

Balslev D, Braet W, McAllister C, Miall RC (2007) Inter-individual variability in optimal current direction for transcranial magnetic stimulation of the motor cortex. Journal of neuroscience methods 162:309-313.

Barker AT, Jalinous R, Freeston IL (1985) Non-invasive magnetic stimulation of human motor cortex. Lancet 1:1106-1107.

Bikson M, Dmochowski J, Rahman A (2013a) The "quasi-uniform" assumption in animal and computational models of non-invasive electrical stimulation. Brain stimulation 6:704705.

Bikson M, Inoue M, Akiyama H, Deans JK, Fox JE, Miyakawa H, Jefferys JG (2004) Effects of uniform extracellular DC electric fields on excitability in rat hippocampal slices in vitro. The Journal of physiology 557:175-190.

Bikson M, Name A, Rahman A (2013b) Origins of specificity during tDCS: anatomical, activity-selective, and input-bias mechanisms. Frontiers in human neuroscience 7:688.

Brasil-Neto JP, McShane LM, Fuhr P, Hallett M, Cohen LG (1992) Topographic mapping of the human motor cortex with magnetic stimulation: factors affecting accuracy and reproducibility. Electroencephalogr Clin Neurophysiol 85:9-16.

Caparelli-Daquer EM, Zimmermann TJ, Mooshagian E, Parra LC, Rice JK, Datta A, Bikson M, Wassermann EM (2012) A pilot study on effects of $4 \times 1$ high-definition tDCS on motor cortex excitability. Conference proceedings : Annual International Conference of the IEEE Engineering in Medicine and Biology Society IEEE Engineering in Medicine and Biology Society Conference 2012:735-738.

Chen M, Mogul DJ (2010) Using increased structural detail of the cortex to improve the accuracy of modeling the effects of transcranial magnetic stimulation on neocortical activation. IEEE transactions on bio-medical engineering 57:1216-1226.

Datta A, Bansal V, Diaz J, Patel J, Reato D, Bikson M (2009) Gyri-precise head model of transcranial direct current stimulation: improved spatial focality using a ring electrode versus conventional rectangular pad. Brain stimulation 2:201-207, 207 e201.

Datta A, Bikson M, Fregni $F$ (2010) Transcranial direct current stimulation in patients with skull defects and skull plates: high-resolution computational FEM study of factors altering cortical current flow. Neuroimage 52:1268-1278.

Datta A, Elwassif M, Battaglia F, Bikson M (2008) Transcranial current stimulation focality using disc and ring electrode configurations: FEM analysis. Journal of neural engineering 5:163-174. 
Day BL, Dressler D, Maertens de Noordhout A, Marsden CD, Nakashima K, Rothwell JC, Thompson PD (1989) Electric and magnetic stimulation of human motor cortex: surface EMG and single motor unit responses. The Journal of physiology 412:449473.

de Berker AO, Bikson M, Bestmann S (2013) Predicting the behavioral impact of transcranial direct current stimulation: issues and limitations. Frontiers in human neuroscience 7:613.

Deans JK, Powell AD, Jefferys JG (2007) Sensitivity of coherent oscillations in rat hippocampus to AC electric fields. The Journal of physiology 583:555-565.

Di Lazzaro V, Restuccia D, Oliviero A, Profice P, Ferrara L, Insola A, Mazzone P, Tonali P, Rothwell JC (1998) Effects of voluntary contraction on descending volleys evoked by transcranial stimulation in conscious humans. The Journal of physiology 508 ( Pt 2):625-633.

Dmochowski JP, Datta A, Bikson M, Su Y, Parra LC (2011) Optimized multi-electrode stimulation increases focality and intensity at target. Journal of neural engineering 8:046011.

Edwards D, Cortes M, Datta A, Minhas P, Wassermann EM, Bikson M (2013) Physiological and modeling evidence for focal transcranial electrical brain stimulation in humans: a basis for high-definition tDCS. Neurolmage 74:266-275.

Fitzgerald PB, Fountain S, Daskalakis ZJ (2006) A comprehensive review of the effects of rTMS on motor cortical excitability and inhibition. Clinical neurophysiology : official journal of the International Federation of Clinical Neurophysiology 117:2584-2596.

Foster KR, Schwan HP (1989) Dielectric properties of tissues and biological materials: a critical review. Critical reviews in biomedical engineering 17:25-104.

Fox PT, Narayana S, Tandon N, Sandoval H, Fox SP, Kochunov P, Lancaster JL (2004) Column-based model of electric field excitation of cerebral cortex. Human brain mapping 22:1-14.

Francis JT, Gluckman BJ, Schiff SJ (2003) Sensitivity of neurons to weak electric fields. The Journal of neuroscience : the official journal of the Society for Neuroscience 23:72557261.

Frohlich F, McCormick DA (2010) Endogenous electric fields may guide neocortical network activity. Neuron 67:129-143.

Heller L, van Hulsteyn DB (1992) Brain stimulation using electromagnetic sources: theoretical aspects. Biophysical journal 63:129-138.

Kuo HI, Bikson M, Datta A, Minhas P, Paulus W, Kuo MF, Nitsche MA (2013) Comparing cortical plasticity induced by conventional and high-definition $4 \times 1$ ring tDCS: a neurophysiological study. Brain stimulation 6:644-648.

Lefaucheur JP, Andre-Obadia N, Poulet E, Devanne H, Haffen E, Londero A, Cretin B, Leroi AM, Radtchenko A, Saba G, Thai-Van H, Litre CF, Vercueil L, Bouhassira D, Ayache SS, Farhat WH, Zouari HG, Mylius V, Nicolier M, Garcia-Larrea L (2011) [French guidelines on the use of repetitive transcranial magnetic stimulation (rTMS): safety and therapeutic indications]. Neurophysiologie clinique $=$ Clinical neurophysiology 41:221-295. 
Legon W, Sato TF, Opitz A, Mueller J, Barbour A, Williams A, Tyler WJ (2014) Transcranial focused ultrasound modulates the activity of primary somatosensory cortex in humans. Nature neuroscience 17:322-329.

López-Alonso V, Cheeran B, Río-Rodríguez D, Fernández-del-Olmo M (2014) Inter-individual Variability in Response to Non-invasive Brain Stimulation Paradigms. BRAIN STIMULATION: Basic, Translational, and Clinical Research in Neuromodulation.

Miranda PC (2013) Physics of effects of transcranial brain stimulation. Handbook of clinical neurology 116:353-366.

Miranda PC, Correia L, Salvador R, Basser PJ (2007) Tissue heterogeneity as a mechanism for localized neural stimulation by applied electric fields. Physics in medicine and biology 52:5603-5617.

Miranda PC, Faria P, Hallett M (2009) What does the ratio of injected current to electrode area tell us about current density in the brain during tDCS? Clinical neurophysiology : official journal of the International Federation of Clinical Neurophysiology 120:11831187.

Miranda PC, Hallett M, Basser PJ (2003) The electric field induced in the brain by magnetic stimulation: a 3-D finite-element analysis of the effect of tissue heterogeneity and anisotropy. IEEE transactions on bio-medical engineering 50:1074-1085.

Miranda PC, Mekonnen A, Salvador R, Ruffini G (2013) The electric field in the cortex during transcranial current stimulation. Neuroimage 70:48-58.

Neuling T, Wagner S, Wolters CH, Zaehle T, Herrmann CS (2012) Finite-Element Model Predicts Current Density Distribution for Clinical Applications of tDCS and tACS. Frontiers in psychiatry 3:83.

Nicholson PW (1965) Specific impedance of cerebral white matter. Experimental neurology 13:386-401.

Nitsche MA, Cohen LG, Wassermann EM, Priori A, Lang N, Antal A, Paulus W, Hummel F, Boggio PS, Fregni F, Pascual-Leone A (2008) Transcranial direct current stimulation: State of the art 2008. Brain stimulation 1:206-223.

Nitsche MA, Paulus W (2000) Excitability changes induced in the human motor cortex by weak transcranial direct current stimulation. The Journal of physiology 527 Pt 3:633639.

Nummenmaa A, McNab JA, Savadjiev P, Okada Y, Hamalainen MS, Wang R, Wald LL, Pascual-Leone A, Wedeen VJ, Raij T (2014) Targeting of white matter tracts with transcranial magnetic stimulation. Brain stimulation 7:80-84.

Nummenmaa A, Stenroos M, Ilmoniemi RJ, Okada YC, Hamalainen MS, Raij T (2013) Comparison of spherical and realistically shaped boundary element head models for transcranial magnetic stimulation navigation. Clinical neurophysiology : official journal of the International Federation of Clinical Neurophysiology 124:1995-2007.

Opitz A, Windhoff M, Heidemann RM, Turner R, Thielscher A (2011) How the brain tissue shapes the electric field induced by transcranial magnetic stimulation. Neurolmage 58:849-859.

Ozen S, Sirota A, Belluscio MA, Anastassiou CA, Stark E, Koch C, Buzsaki G (2010) Transcranial electric stimulation entrains cortical neuronal populations in rats. The 
Journal of neuroscience : the official journal of the Society for Neuroscience 30:11476-11485.

Padberg F, George MS (2009) Repetitive transcranial magnetic stimulation of the prefrontal cortex in depression. Experimental neurology 219:2-13.

Pascual-Leone A, Bartres-Faz D, Keenan JP (1999) Transcranial magnetic stimulation: studying the brain-behaviour relationship by induction of 'virtual lesions'. Philosophical transactions of the Royal Society of London Series B, Biological sciences 354:12291238.

Pashut T, Wolfus S, Friedman A, Lavidor M, Bar-Gad I, Yeshurun Y, Korngreen A (2011) Mechanisms of magnetic stimulation of central nervous system neurons. PLoS computational biology 7:e1002022.

Patton HD, Amassian VE (1954) Single and multiple-unit analysis of cortical stage of pyramidal tract activation. Journal of neurophysiology 17:345-363.

Paulus W (2011) Transcranial electrical stimulation (tES - tDCS; tRNS, tACS) methods. Neuropsychological rehabilitation 21:602-617.

Plonsey R, Heppner DB (1967) Considerations of quasi-stationarity in electrophysiological systems. The Bulletin of mathematical biophysics 29:657-664.

Radman T, Ramos RL, Brumberg JC, Bikson M (2009) Role of cortical cell type and morphology in subthreshold and suprathreshold uniform electric field stimulation in vitro. Brain stimulation 2:215-228, 228 e211-213.

Radman T, Su Y, An JH, Parra LC, Bikson M (2007) Spike timing amplifies the effect of electric fields on neurons: implications for endogenous field effects. The Journal of neuroscience : the official journal of the Society for Neuroscience 27:3030-3036.

Rahman A, Reato D, Arlotti M, Gasca F, Datta A, Parra LC, Bikson M (2013) Cellular effects of acute direct current stimulation: somatic and synaptic terminal effects. The Journal of physiology 591:2563-2578.

Rampersad SM, Stegeman DF, Oostendorp TF (2013) Single-layer skull approximations perform well in transcranial direct current stimulation modeling. IEEE transactions on neural systems and rehabilitation engineering : a publication of the IEEE Engineering in Medicine and Biology Society 21:346-353.

Ranck JB, Jr., Bement SL (1965) The specific impedance of the dorsal columns of cat: An inisotropic medium. Experimental neurology 11:451-463.

Rathelot JA, Strick PL (2009) Subdivisions of primary motor cortex based on corticomotoneuronal cells. Proceedings of the National Academy of Sciences of the United States of America 106:918-923.

Rattay F (1986) Analysis of models for external stimulation of axons. IEEE Trans Biomed Eng 33:974-977.

Reato D, Gasca F, Datta A, Bikson M, Marshall L, Parra LC (2013) Transcranial electrical stimulation accelerates human sleep homeostasis. PLoS computational biology 9:e1002898. 
Reato D, Rahman A, Bikson M, Parra LC (2010) Low-intensity electrical stimulation affects network dynamics by modulating population rate and spike timing. The Journal of neuroscience : the official journal of the Society for Neuroscience 30:15067-15079.

Richter L, Neumann G, Oung S, Schweikard A, Trillenberg P (2013) Optimal coil orientation for transcranial magnetic stimulation. PloS one 8:e60358.

Rotem A, Neef A, Neef NE, Agudelo-Toro A, Rakhmilevitch D, Paulus W, Moses E (2014) Solving the orientation specific constraints in transcranial magnetic stimulation by rotating fields. PloS one 9:e86794.

Roth BJ, Saypol JM, Hallett M, Cohen LG (1991) A theoretical calculation of the electric field induced in the cortex during magnetic stimulation. Electroencephalogr Clin Neurophysiol 81:47-56.

Rothwell JC, Hallett M, Berardelli A, Eisen A, Rossini P, Paulus W (1999) Magnetic stimulation: motor evoked potentials. The International Federation of Clinical Neurophysiology. Electroencephalography and clinical neurophysiology Supplement 52:97-103.

Ruffini G, Fox MD, Ripolles O, Miranda PC, Pascual-Leone A (2014) Optimization of multifocal transcranial current stimulation for weighted cortical pattern targeting from realistic modeling of electric fields. Neurolmage 89:216-225.

Ruohonen J, Karhu J (2010) Navigated transcranial magnetic stimulation. Neurophysiologie clinique $=$ Clinical neurophysiology 40:7-17.

Rush S, Driscoll DA (1968) Current distribution in the brain from surface electrodes. Anesthesia and analgesia 47:717-723.

Sadleir RJ, Vannorsdall TD, Schretlen DJ, Gordon B (2010) Transcranial direct current stimulation (tDCS) in a realistic head model. Neuroimage 51:1310-1318.

Salinas FS, Lancaster JL, Fox PT (2009) 3D modeling of the total electric field induced by transcranial magnetic stimulation using the boundary element method. Physics in medicine and biology 54:3631-3647.

Salminen-Vaparanta N, Noreika V, Revonsuo A, Koivisto M, Vanni S (2012) Is selective primary visual cortex stimulation achievable with TMS? Human Brain Mapping 33:652-665.

Salvador R, Mekonnen A, Ruffini G, Miranda PC (2010) Modeling the electric field induced in a high resolution realistic head model during transcranial current stimulation. Conference proceedings: Annual International Conference of the IEEE Engineering in Medicine and Biology Society IEEE Engineering in Medicine and Biology Society Conference 2010:2073-2076.

Salvador R, Silva S, Basser PJ, Miranda PC (2011) Determining which mechanisms lead to activation in the motor cortex: a modeling study of transcranial magnetic stimulation using realistic stimulus waveforms and sulcal geometry. Clinical neurophysiology : official journal of the International Federation of Clinical Neurophysiology 122:748758.

Suh HS, Lee WH, Kim TS (2012) Influence of anisotropic conductivity in the skull and white matter on transcranial direct current stimulation via an anatomically realistic finite element head model. Physics in medicine and biology 57:6961-6980. 
Thielscher A, Kammer T (2002) Linking physics with physiology in TMS: a sphere field model to determine the cortical stimulation site in TMS. Neurolmage 17:1117-1130.

Thielscher A, Kammer T (2004) Electric field properties of two commercial figure-8 coils in TMS: calculation of focality and efficiency. Clinical neurophysiology : official journal of the International Federation of Clinical Neurophysiology 115:1697-1708.

Thielscher A, Opitz A, Windhoff M (2011) Impact of the gyral geometry on the electric field induced by transcranial magnetic stimulation. Neurolmage 54:234-243.

Toschi N, Welt T, Guerrisi M, Keck ME (2008) A reconstruction of the conductive phenomena elicited by transcranial magnetic stimulation in heterogeneous brain tissue. Physica Medica 24:80-86.

Tuch DS, Wedeen VJ, Dale AM, George JS, Belliveau JW (2001) Conductivity tensor mapping of the human brain using diffusion tensor MRI. Proceedings of the National Academy of Sciences of the United States of America 98:11697-11701.

Tufail Y, Matyushov A, Baldwin N, Tauchmann ML, Georges J, Yoshihiro A, Helms Tillery SI, Tyler WJ (2010) Transcranial Pulsed Ultrasound Stimulates Intact Brain Circuits Neuron 66:681-694.

Wagner S, Rampersad SM, Aydin U, Vorwerk J, Oostendorp TF, Neuling T, Herrmann CS, Stegeman DF, Wolters CH (2013) Investigation of tDCS volume conduction effects in a highly realistic head model. Journal of neural engineering 11:016002.

Wang W, Eisenberg SR (1994) A three-dimensional finite element method for computing magnetically induced currents in tissues. Magnetics, IEEE Transactions on 30:50155023.

Wiethoff S, Hamada M, Rothwell JC (2014) Variability in response to transcranial direct current stimulation of the motor cortex. Brain Stimulation: Basic, Translational, and Clinical Research in Neuromodulation.

Windhoff M, Opitz A, Thielscher A (2013) Electric field calculations in brain stimulation based on finite elements: an optimized processing pipeline for the generation and usage of accurate individual head models. Hum Brain Mapp 34:923-935. 


\section{Acknowledgements}

First, I want to thank Prof. Walter Paulus for giving me the opportunity to pursue a PhD under his supervision in his department. His great enthusiasm for developing new brain stimulation methods was a constant source of ideas for exciting projects. I am also very grateful for the freedom he gave me to explore my various different scientific interests during my PhD.

I want to thank the members of my thesis committee Prof. Florentin Wörgötter and Prof. Jens Frahm for their scientific advice and guidance. I am grateful to the team of the GGNB and the PTCN program, in particular Antje Erdmann and Kerstin Mosch, who were very supportive with bureaucratic problems.

Furthermore, I want to thank my scientific collaborators with whom I could work on many interesting projects. My sincere gratitude goes to Axel Thielscher, now at the University of Copenhagen, who already introduced me to the field of brain stimulation and numerical modelling as an undergraduate student. Also, the common development of numerical models optimized for tDCS led to many interesting new insights. Second, I want to thank Jamie Tyler from the Virginia Tech Carillion Research Institute in Roanoke, VA who introduced me to the exciting technique of transcranial focused ultrasound stimulation and who was a great mentor for me in my scientific development. My heartfelt thanks go as well to my colleague and friend Wynn Legon who taught me most of what I know about the experimental aspects of brain stimulation. Finally, I want to thank Noman Zafar, Volker Bockermann and Veit Rohde from the department of Neurosurgery for the productive collaboration and the exciting possibility to test my models in brain tumor patients.

Also I want to thank my colleagues and friends in the department including Zsolt Turi, Catarina Saiote, Giorgi Batzikdaze, Andrea Antal, Patrik Kunz, Ivan Alekseichuk, Dona Kyprianou, Leonie Schmalfuss, Shane Fresnoza, Manuel Hewitt, Alberto Pisoni, Asif Jamil, Yuichiro Shirota, Kay Reimers and many others for interesting talks during lunch, collaboration in various projects and creating a fun working atmosphere. Also I want to thank Marion Kurze for her help with bureaucratic issues.

Last but not least, I want to thank my family and especially my girlfriend for their continuing practical help as well as emotional support during my PhD time. 


\section{Education}

Since January 2012

February 2011

October 2008

October 2006
PhD student in Theoretical and Computational Neuroscience at the University of Göttingen, department of clinical neurophysiology University of Tuebingen, Diploma in physics University of Tuebingen, Prediploma in psychology University of Bayreuth, Prediploma in physics

\section{Work Experience}

June 2011 - December 2011

visiting scholar at the Virginia Tech Carillon Research Institute in Roanoke, VA, USA

November 2009 - February 2011 research assistant at the High-Field Magnetic Resonance Center at the Max Planck Institute in Tuebingen

\section{Publications}

Thielscher A, Opitz A, Windhoff M (2011) Impact of the gyral geometry on the electric field induced by transcranial magnetic stimulation. Neurolmage 54:234-243.

Opitz A, Windhoff M, Heidemann RM, Turner R, Thielscher A (2011) How the brain tissue shapes the electric field induced by transcranial magnetic stimulation. Neurolmage 58:849859.

Windhoff M, Opitz A, Thielscher A (2013) Electric field calculations in brain stimulation based on finite elements: an optimized processing pipeline for the generation and usage of accurate individual head models. Hum Brain Mapping 34:923-935.

Legon W, Rowlands A, Opitz A, Sato TF, Tyler WJ (2012) Pulsed ultrasound differentially stimulates somatosensory circuits in humans as indicated by EEG and FMRI. PloS One 
Paulus W, Opitz A (2013) Ohm's law and tDCS over the centuries. Clinical neurophysiology: official journal of the International Federation of Clinical Neurophysiology 124:429-430.

Opitz A, Legon W, Rowlands A, Bickel WK, Paulus W, Tyler WJ (2013) Physiological observations validate finite element models for estimating subject-specific electric field distributions induced by transcranial magnetic stimulation of the human motor cortex. Neurolmage 81:253-264.

Chaieb L, Antal A, Pisoni A, Saiote C, Opitz A, Ambrus GG, Focke N, Paulus W. (2014) Safety of $5 \mathrm{kHz}$ tACS. Brain Stimulation 7:92-6.

Legon W, Sato TF, Opitz A, Mueller J, Barbour A, Williams A, Tyler WJ (2014) Transcranial focused ultrasound modulates the activity of primary somatosensory cortex in humans. Nature Neuroscience 17:322-9.

Saiote C, Goldschmidt T, Timäus C, Steenwijk M.D., Opitz A, Antal A, Paulus W, Nitsche M. Impact of transcranial direct current stimulation on fatigue in multiple sclerosis. Restorative Neurology and Neuroscience (in press)

Opitz A*, Zafar N*, Bockermann V, Rohde V, Paulus W. Validating computationally predicted TMS stimulation areas using direct electrical stimulation in patients with brain tumors near precentral regions. (in press in Neuroimage: Clinical)

Opitz A, Paulus W, Will S, Thielscher A. Anatomical determinants of the electric field during transcranial direct current stimulation. (in revision)

Turi Z*, Opitz A*, Mittner M*, Popkes M, Sengupta T, Paulus W, Antal A. Towards functional differentiation between prefrontal and striatal components of instrumental learning by prefrontal transcranial direct current stimulation. (in revision)

Opitz A, Legon W, Mueller J, Barbour A, Williams A, Bickel WK, Paulus W, Tyler WJ. Sham transcranial magnetic stimulation of the dorsal lateral prefrontal cortex induces plasticity. (submitted) 
Posters and presentations (only first author)

Opitz, A., Windhoff, M., Reichenbach, A. and Thielscher, A. (2010): Calculation of the electric field induced by TMS: Influence of white matter anisotropy, Magstim/University of Oxford TMS Summer School, 2010.

Opitz, A., Windhoff, M., Heidemann, R., Turner, R. and Thielscher, A.: How do Gyral Orientation and White Matter Anisotropy Affect the Electric Field Induced by TMS?, HBM 2011, Quebec City. (oral presentation, trainee abstract award).

Opitz, A., Legon, W., Rowlands, A., Paulus, W. and Tyler, W.J.: Neurophysiological validation of finite element models used to predict cortical excitation volumes generated by transcranial magnetic stimulation in humans, FENS 2012, Barcelona.

Opitz, A., Legon, W., Rowlands, A., Paulus, W. and Tyler, W.J.: Neurophysiological validation of finite element models used to predict cortical excitation volumes generated by transcranial magnetic stimulation in humans, 10th Göttingen Meeting of the German Neuroscience Society 2013, Göttingen.

Opitz, A., Legon, W., Rowlands, A., Paulus, W. and Tyler, W.J.: Neurophysiological validation of finite element models used to predict cortical excitation volumes generated by transcranial magnetic stimulation in humans, 5th International Conference on Non-invasive Brain Stimulation 2013, Leipzig.

Opitz, A., Legon, W., Mueller, J. , Barbour, A., Williams, A., Paulus, W., Tyler, W.J.: Effects of the residual electric field induced by sham coils should not be neglected in TMS studies, HBM 2013, Seattle

Opitz, A., Paulus' W., Will' S., Thielscher, A.: Where do we stimulate with tDCS? Anatomical determinants of the electric field during transcranial direct current stimulation, ICCN 2014, Berlin 